\title{
DESIGN, ANALYSIS \& TESTING OF AN ENHANCED RADIAL-AXIAL HYBRID COMPLIANT DEBURRING TOOL
}

by

\author{
Jeremy Kroeker \\ Bachelor of Aerospace Engineering \\ Ryerson University \\ 2010

\begin{abstract}
A thesis
presented to Ryerson University

in partial fulfillment of the

requirements for the degree of

Master of Applied Science

in the Program of

Aerospace Engineering
\end{abstract}

Toronto, Ontario, Canada, 2015

(C) Jeremy Kroeker 2015 


\title{
Design, Analysis \& Testing of an Enhanced Radial-Axial Hybrid Compliant Deburring Tool
}

\author{
Jeremy Kroeker \\ Master of Applied Science, 2015 \\ Aerospace Engineering \\ Ryerson University
}

\section{Abstract}

This thesis will discuss the development of a radial actuator incorporated into a deburring tool.

Gas turbine engine deburring is complex; this requires the tooltip to maintain active compliance in three degrees of freedom. This can be achieved through the use of a rotating action plane so that only radial and axial actuation is required. A proposed enhanced radial actuator has been made that utilizes the action plane model and fulfill the requirements for precision deburring of gas turbine engine components. The enhanced radial actuator was designed using four silicone rubber pneumatic diaphragms. The diaphragms were modelled using a finite element method and

applying an Arruda-Boyce material model to the mesh. The stiffness behaviour was analyzed and compared to data from previous research completed on radial actuation within an action plane. The stiffness behaviour was determined to be superior and significantly improved as it could be reliably predicted. 


\section{Acknowledgments}

I would like to thank my supervisor Dr. Jeff Xi for all of his guidance and support throughout the length of my graduate studies. I couldn't have made it this far without the love and support of my spouse Catherine and family. As well I would like to thank Mr. Brian Petz and Mr. Primoz Crensnik for all of their support and advice. Thanks are also extended to Dr. Hamid Ghaemi, Mr.

Peter Bradley, Mr. Michael Rososhansky, Mr. Vangjel Pano, Mr. Yu Lin, and Mr. Richard Mohamed. 


\section{Table of Contents}

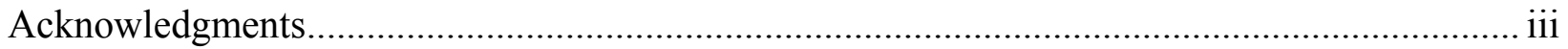

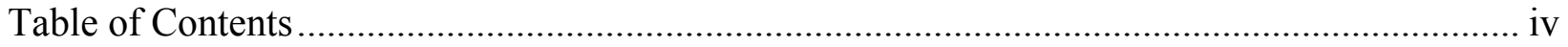

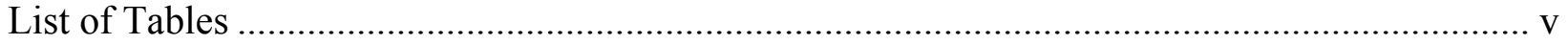

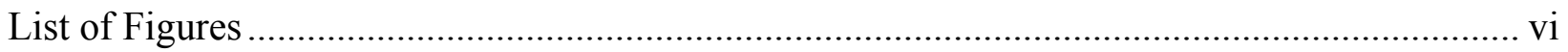

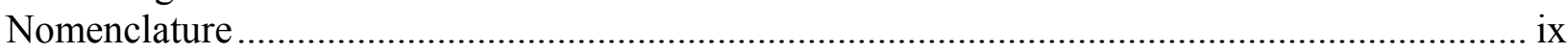

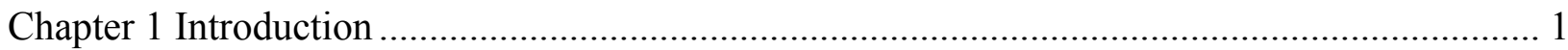

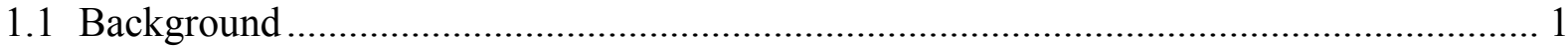

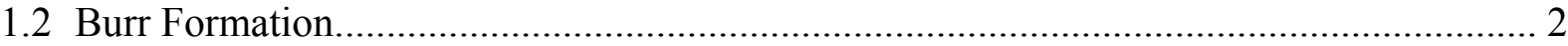

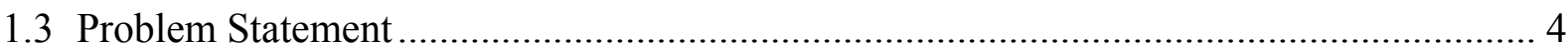

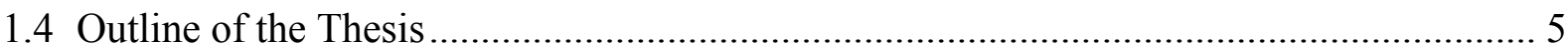

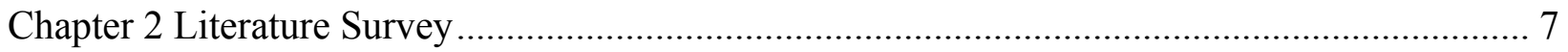

2.1 Burr Removal ..................................................................................................... 7

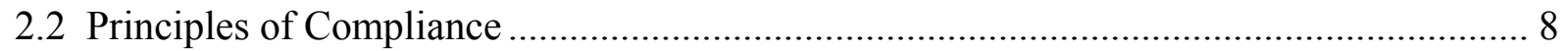

2.3 Existing Methods for Automated Deburring .............................................................. 9

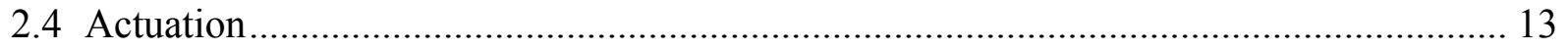

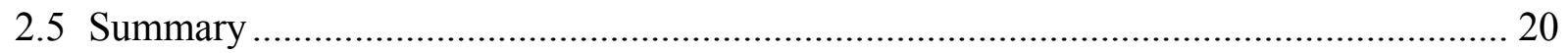

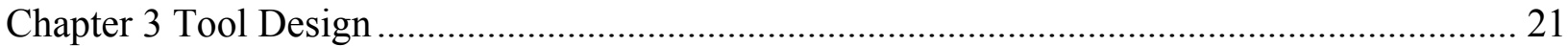

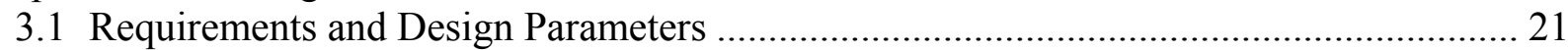

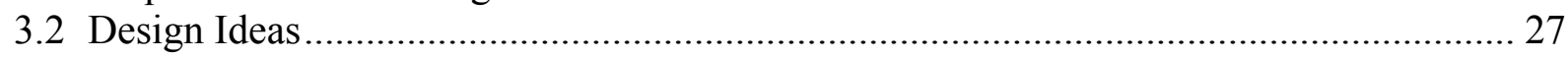

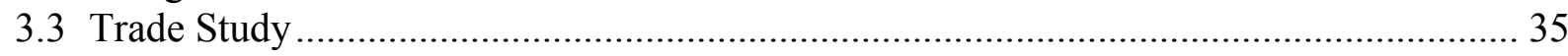

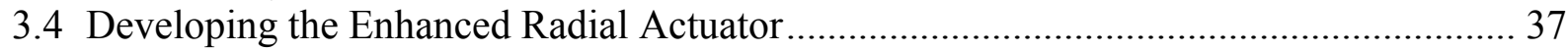

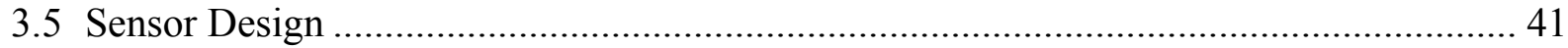

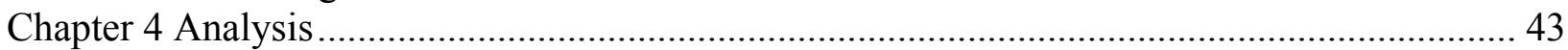

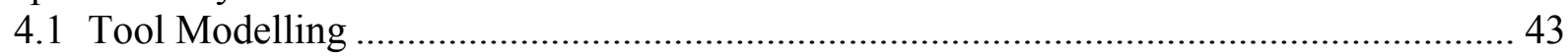

4.2 Tool and Workpiece Interaction .............................................................................. 48

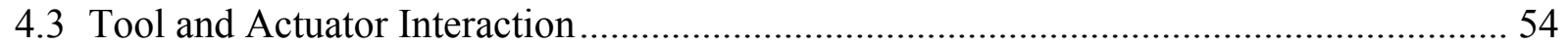

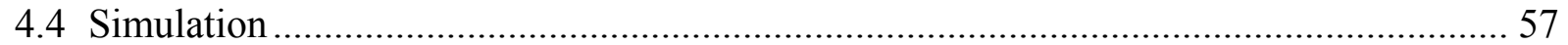

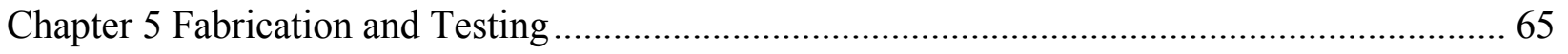

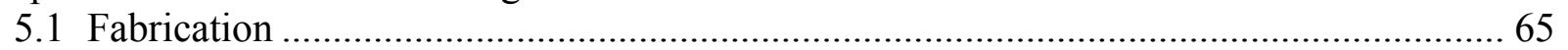

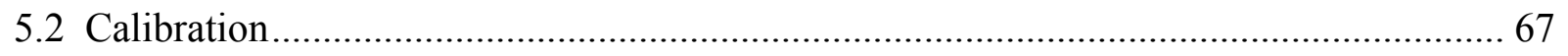

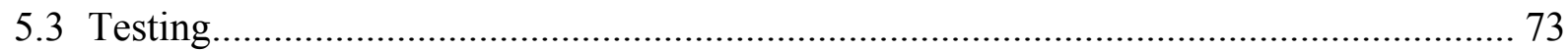

Chapter 6 Conclusion and Future Work ……………....................................................... 80

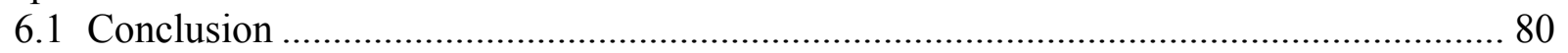

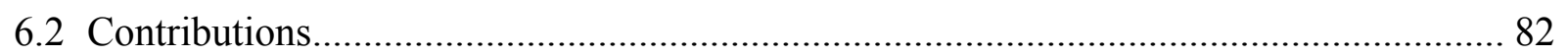

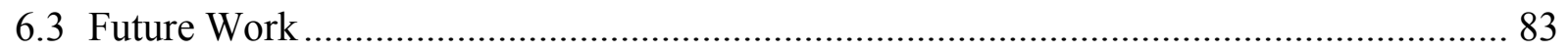

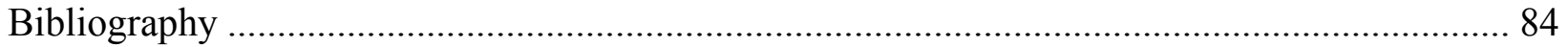

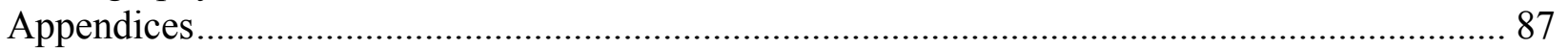




\section{List of Tables}

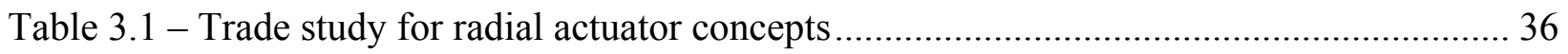




\section{List of Figures}

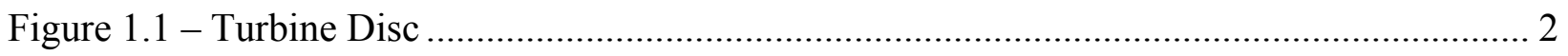

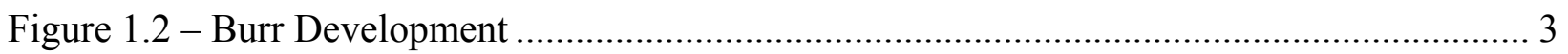

Figure 1.3 - Four Main Burr Types ..................................................................................... 4

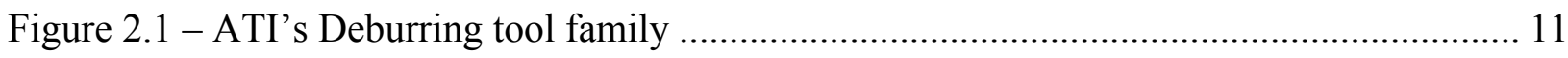

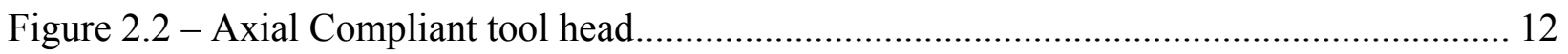

Figure 2.3 - Radial-axial hybrid active force compliant deburring tool head (HFCDT) ............. 13

Figure 2.4 - TriKinetics Adaptive Deburring Tool ............................................................... 14

Figure 2.5 - United Technologies Research Center (UTRC) Chamfer and Deburring

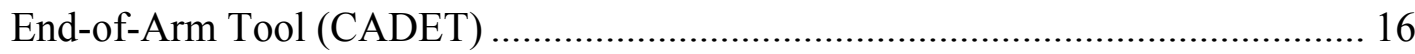

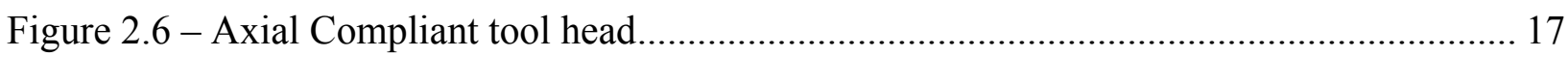

Figure 2.7 - ATI ring actuator assembly on the Flexdeburr tool............................................... 18

Figure 2.8 - PRA bicycle inner tube stretched around a conduit ring ......................................... 19

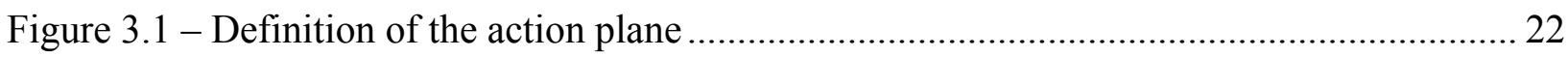

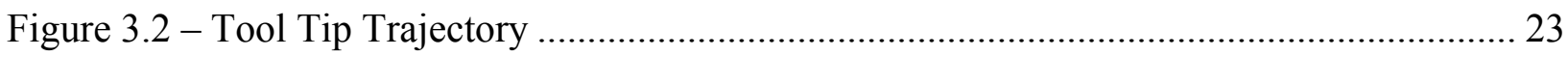

Figure 3.3 - Deburring tool model within action plane ............................................................ 24

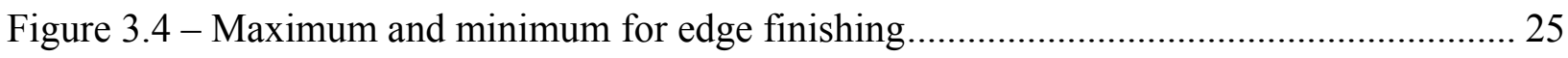

Figure 3.5 - HFCDT PRA cross-section and isometric view .................................................... 27

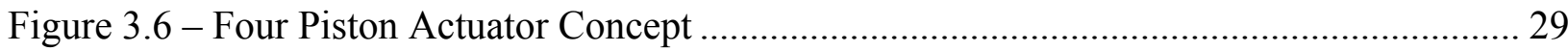

Figure 3.7 - Eight Piston Actuator Concept …………………................................................ 30

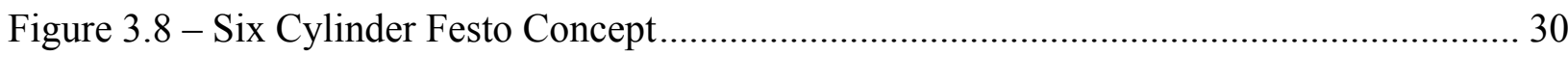

Figure 3.9 - Cross-section of wrap-around diaphragm concept .................................................. 31

Figure 3.10 - Four, five, and six chamber wrap-around diaphragm concepts.............................. 32

Figure 3.11 - Single chamber Diaphragm with Inlaying Retaining Rings Concept .................... 33

Figure 3.12 - Single Chamber Diaphragm with Interference Retaining Rings Concept............... 33

Figure 3.13 - Four Chamber Diaphragm with Window Retainers Concept.................................. 34

Figure 3.14 - Four Chamber diaphragm with retainer Clips Concept.......................................... 35

Figure 3.15 - Cross-section of inflated diaphragm contacting tool shaft ..................................... 37

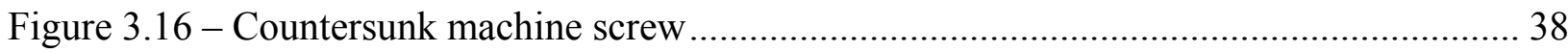

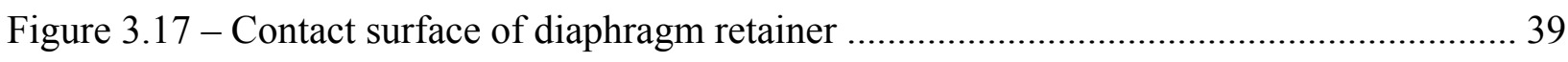

Figure 3.18 - Mockup of rubber diaphragm ............................................................................ 41

Figure 4.1 - Global coordinate system of the deburring tool .................................................... 44 
Figure 4.2 - Model of deburring tool in action plane ........................................................... 45

Figure 4.3 - Hertzian disc contact geometry ................................................................... 49

Figure 4.4 - Hertzian disc contact tangent plane .............................................................. 50

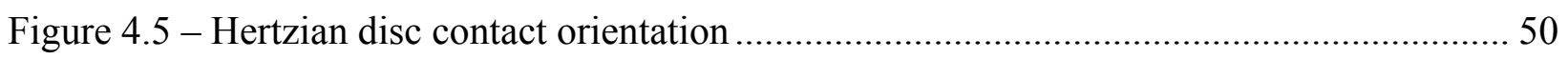

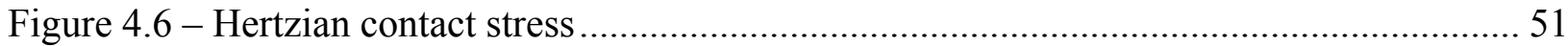

Figure 4.7 - Mean stress distribution across elliptical contact area ....................................... 53

Figure 4.8 - Eight-chain network model ................................................................... 55

Figure 4.9 - Initial diaphragm/tool model .................................................................. 58

Figure 4.10 - Symmetry on the tool shaft/diaphragm model ............................................ 58

Figure 4.11 - Tool shaft/diaphragm contact condition ..................................................... 59

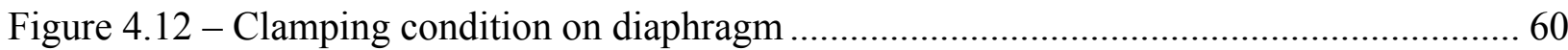

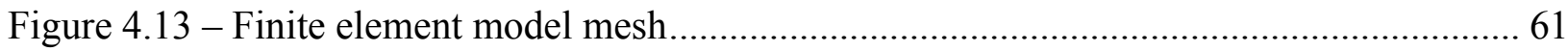

Figure 4.14 - Pressure and force conditions on model .................................................... 61

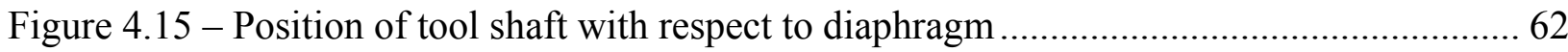

Figure 4.16 - Total displacement of ANSYS finite element model ....................................... 63

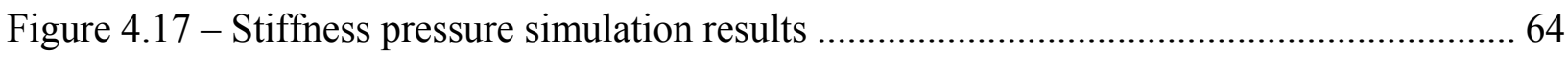

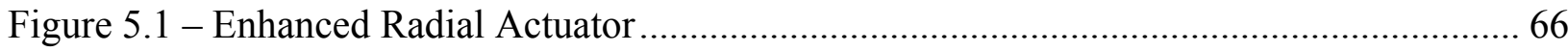

Figure 5.2 - Assembly of the enhanced radial actuator .................................................... 67

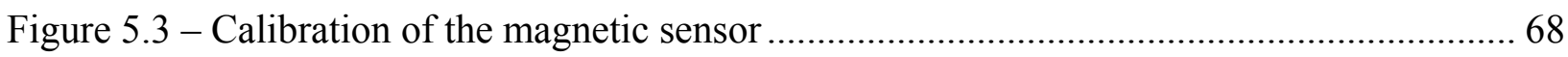

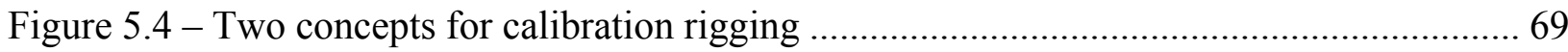

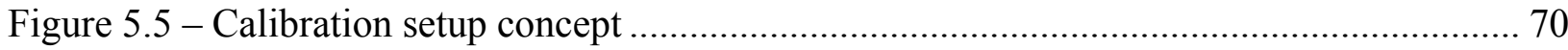

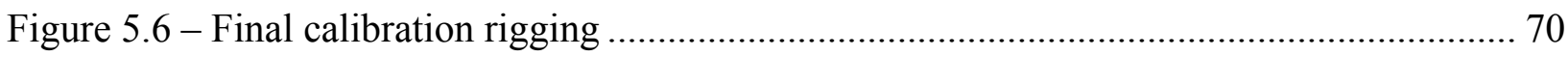

Figure 5.7 - Simulink Program for Calibration ............................................................... 71

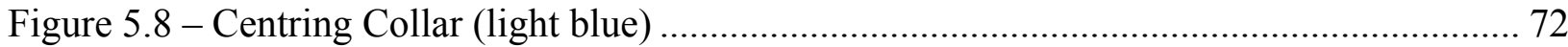

Figure 5.9 - Voltage vs. Displacement Relationship ................................................... 73

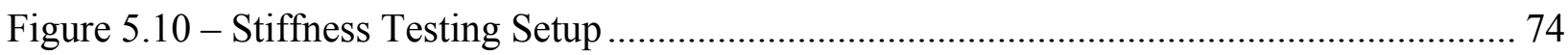

Figure 5.11 - Experimental stiffness and pressure testing results .................................... 75

Figure 5.12 - Stiffness vs. pressure relationship for simulation and testing data..................... 76

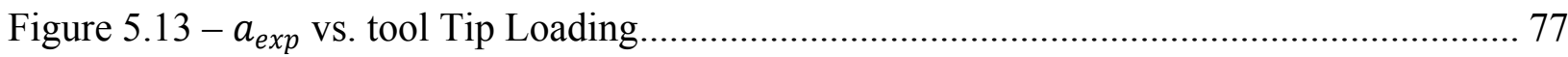

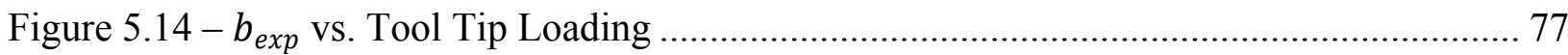

Figure 5.15 - Stiffness behaviour between the PRA and ERA ........................................... 78

Figure A-1 Results for 1N shaft force and 100MPa diaphragm pressure............................... 87 
Figure A-2 Results for 1N shaft force and 150MPa diaphragm pressure.................................. 87

Figure A-3 Results for $1 \mathrm{~N}$ shaft force and 200MPa diaphragm pressure................................ 88

Figure A-4 Results for $1 \mathrm{~N}$ shaft force and 250MPa diaphragm pressure.................................. 88

Figure A-5 Results for 1.5N shaft force and 100MPa diaphragm pressure............................. 89

Figure A-6 Results for 1.5N shaft force and 150MPa diaphragm pressure ............................. 89

Figure A-7 Results for 1.5N shaft force and 210MPa diaphragm pressure ............................. 90

Figure A-8 Results for 1.5N shaft force and 260MPa diaphragm pressure ............................. 90

Figure A-9 Results for 1.5N shaft force and 280MPa diaphragm pressure ............................ 91

Figure A-10 Results for 1.5N shaft force and 300MPa diaphragm pressure .......................... 91

Figure A-11 Results for 1.5N shaft force and 400MPa diaphragm pressure .......................... 92

Figure A-12 Results for 2N shaft force and 100MPa diaphragm pressure ............................. 92

Figure A-13 Results for 2N shaft force and 150MPa diaphragm pressure ............................. 93

Figure A-14 Results for 2N shaft force and 180MPa diaphragm pressure ............................. 93

Figure A-15 Results for 3N shaft force and 100MPa diaphragm pressure ............................. 94

Figure A-16 Results for 3N shaft force and 150MPa diaphragm pressure ............................. 94

Figure A-17 Results for 3N shaft force and 170MPa diaphragm pressure ............................. 95

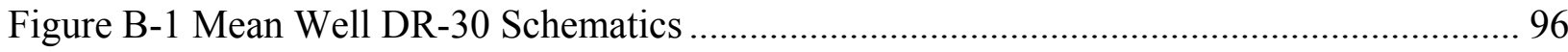

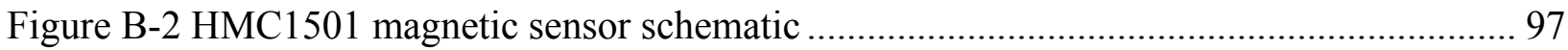

Figure B-3 HFCDT position sensing hardware schematics................................................ 98

Figure B-4 PX319-100A5V pressure transducer schematics ................................................ 99

Figure B-5 Measurement Computing USB120FS pin location ........................................ 100

Figure B-6 Measurement Computing USB120FS schematics............................................. 100

Figure C-1 Uniaxial test data from ANSYS ................................................................. 101

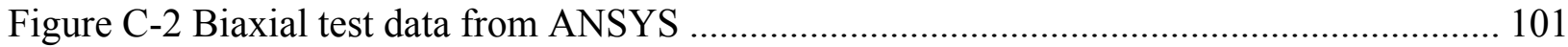

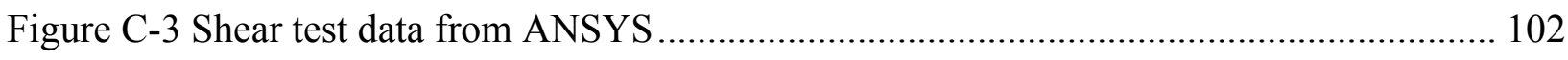

Figure C-4 Volumetric test data from ANSYS ......................................................... 102 


\section{Nomenclature}

\begin{tabular}{|c|c|}
\hline$a$ & Major axis of Hertzian contact ellipse \\
\hline$a_{\text {exp }}$ & First coefficient in standard exponential equation \\
\hline$A$ & Root of a quadratic equation in Hertzian disc contact \\
\hline ADT & Adaptive Deburring Tool \\
\hline AMR & Anisotropic Magneto-Resistant \\
\hline$b$ & Minor axis of Hertzian contact ellipse \\
\hline$b_{l}$ & Length of tool shaft from gimbal axis to tool tip \\
\hline$b_{\text {exp }}$ & Second coefficient in standard exponential equation \\
\hline$b_{u}$ & Length of tool shaft from gimbal to actuator \\
\hline$B$ & Root of a quadratic equation in Hertzian disc contact \\
\hline$B_{i j}$ & Left Cauchy-Green deformation tensor \\
\hline$B_{k k}$ & First invariant of the left Cauchy-Green deformation tensor \\
\hline CADET & Chamfering and Deburring End-of-arm Tool \\
\hline$C_{i}$ & Right Cauchy-Green deformation tensor \\
\hline$d$ & Total minimum displacement between corresponding points \\
\hline$d_{\text {simulation }}$ & Displacement from simulation \\
\hline$E_{1}$ & Young's Modulus for Hertzian disc one \\
\hline$E_{2}$ & Young's Modulus for Hertzian disc two \\
\hline$E\left(k^{\prime}\right)$ & Elliptical integral \\
\hline ERA & Enhanced Radial Actuator \\
\hline$F$ & Contact force \\
\hline$F_{R A}$ & Force on the tool shaft due to the radial actuator \\
\hline$F_{r-C u t}$ & Force on the tool shaft due to the radial cutting force \\
\hline HFCDT & Hybrid Force Compliant Deburring Tool \\
\hline I & Moment of inertia \\
\hline$I_{1}$ & First invariant of the left Cauchy-Green deformation tensor \\
\hline$J$ & Jacobian determinant of the deformation gradient tensor \\
\hline$k$ & Hertzian contact elliptical ratio \\
\hline$k^{\prime}$ & Derivative of Hertzian contact elliptical ratio \\
\hline$k_{B}$ & Boltzmann constant \\
\hline$K$ & Bulk Modulus \\
\hline$K_{r}$ & Stiffness of the radial actuator \\
\hline$K_{Z}$ & Stiffness of axial actuation of tool shaft \\
\hline$m_{p}$ & Maximum possible burr proximity score \\
\hline$m_{s}$ & Maximum possible burr size score \\
\hline$m_{t}$ & Maximum possible burr tolerance score \\
\hline$m_{z}$ & Mass of the axial actuating tool shaft \\
\hline$M$ & Hertzian ratio \\
\hline$M_{\text {Cut }}$ & Total pivoting moment of the tool shaft due to the radial cutting force \\
\hline$M_{R A}$ & Total pivoting moment of the tool shaft due to the radial actuator \\
\hline MMR & Material Removal Rate \\
\hline$M_{P I V O T}$ & Total pivoting moment of tool shaft about the gimbal axis \\
\hline$n$ & Hertzian ratio \\
\hline$n_{\text {chain }}$ & Number of chain segments \\
\hline$N$ & Number of chains in the network of a cross-linked polymer \\
\hline
\end{tabular}




\begin{tabular}{|c|c|}
\hline$P_{0}$ & Contact pressure \\
\hline$P_{m}$ & Mean stress \\
\hline PRA & Pneumatic Ring Actuator \\
\hline$P_{\text {seg }}(x, y)$ & Elliptical stress distribution \\
\hline $\mathrm{p}$ & Dynamic pressure within the radial actuator \\
\hline$r_{l}$ & Radial displacement of tool tip \\
\hline$\dot{r}_{l}$ & Radial velocity of tool tip \\
\hline$\ddot{r}_{l}$ & Radial acceleration of tool tip \\
\hline$r_{u}$ & Radial displacement of tool head \\
\hline$\dot{r}_{u}$ & Radial velocity of tool head \\
\hline$\ddot{r_{u}}$ & Radial acceleration of tool head \\
\hline$R_{1}$ & Radius of curvature of Hertzian disc one \\
\hline$R_{1}^{\prime}$ & Edge radius of Hertzian disc one \\
\hline$R_{2}$ & Radius of curvature of Hertzian disc two \\
\hline$R_{2}^{\prime}$ & Edge radius of Hertzian disc two \\
\hline$T$ & Temperature \\
\hline $\bar{U}$ & $\begin{array}{l}\text { Strain energy density in terms of the left Cauchy-Green deformation tensor } \\
\text { invariants }\end{array}$ \\
\hline UTRC & United Technologies Research Center \\
\hline$V_{x}$ sensor & Voltage from x sensor \\
\hline$V_{y}$ sensor & Voltage from y sensor \\
\hline$W_{8 c h}$ & Strain energy density for eight chain Arruda-Boyce model \\
\hline$x$ & Cartesian $\mathrm{x}$-axis coordinate \\
\hline$X$ & Complexity factor \\
\hline$y$ & Cartesian y-axis coordinate \\
\hline$Z$ & Axial displacement \\
\hline$\ddot{z}$ & Axial acceleration \\
\hline$z_{1}$ & Point one on Hertzian disc contact \\
\hline$z_{2}$ & Point two on Hertzian disc contact \\
\hline$Z$ & Depth from the contact surface area along the $\mathrm{z}$-axis \\
\hline
\end{tabular}

$\begin{array}{cl}\beta & \text { Inverse of the number of chains in the network of a cross-linked polymer } \\ \delta_{i j} & \text { Kronecker delta } \\ \theta & \text { Angle of shaft from gimbal axis within action plane } \\ \dot{\theta} & \text { Angular velocity of tool shaft } \\ \ddot{\theta} & \text { Angular acceleration of tool shaft } \\ \lambda_{\text {chain }} & \text { A function of the first invariant of the left Cauchy-Green deformation tensor } \\ \lambda_{m} & \text { Locking stretch of a material } \\ \Lambda & \text { A term for the elastic properties and shape of two Hertzian discs in contact } \\ \mu & \text { Initial shear modulus } \\ v_{1} & \text { Poisson's ratio for Hertzian disc one } \\ v_{2} & \text { Poisson's ratio for Hertzian disc two } \\ \sigma_{i j} & \text { Stress-strain function for a given strain energy density } \\ \sigma_{z z} & \text { Principle stress on the contact surface } \\ \phi & \text { Hertzian disc contact angle }\end{array}$




\section{Chapter 1}

\section{Introduction}

\subsection{Background}

Manufacturing turbine engine parts can be a high cost and risk endeavor, due to the tight tolerances required of the complex machined and cast components. The entire value of the part hinges on the part finish quality. If the quality is not within specification and rework cannot be completed to bring it within the desired tolerance the part is considered scrap, resulting in overall increased part cost. At Pratt \& Whitney bench deburring and chamfering is attributed to $12 \%$ of total monthly machining hours [1]. In addition, there is the added cost of very skilled workers to hand deburr each individual part. These skilled workers use tools that are also used in jewel and dental industry to remove burrs and polish the surfaces of the turbine engine parts. This process takes a long time which creates a bottle neck in production. The rework and scrap rate of these parts at this stage of the process increases due to operator errors, as it is very subjective to the operator's skill level and part condition. Many of the tasks to be carried out with regards to finishing are on blind edges in tight proximity to other features on the workpiece. Figure 1.1 illustrates a gas turbine engine disc, typical of a complex hand deburring workpiece. The sum of these factors causes a very difficult and tedious job for those who are tasked with deburring turbine engine parts. 


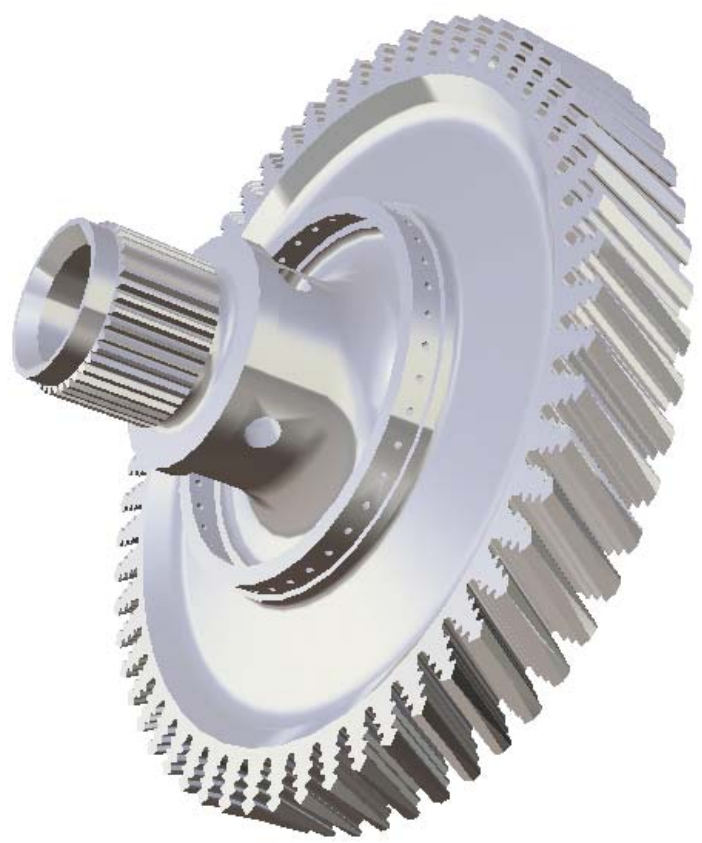

Figure 1.1 - Turbine Disc

A skilled operator who manually deburrs turbine engine parts adapts to complex part topology. When performed effectively the operator exhibits active compliance with the workpiece. Compliance is the ability of a tool to maintain contact and cutting force with the workpiece.

\subsection{Burr Formation}

Burrs are unintentionally created throughout the manufacturing process of parts. They are subjective features on a workpiece when either too much material has been removed or remains. Machining of metals is most often a form of milling or lathing. In the process of milling or lathing, a rigid tool is used to remove the softer material from a workpiece. The tool is plunged into the workpiece with a great amount of force and power to remove material at a rate referred to as the material removal rate (MRR). Burr location is usually predictable as they usually occur at either the entrance or exit of the tool into the workpiece. Plastic deformation of the workpiece usually results in a positive burr, whereas breakage results in negative burr formation. See Figure 1.2 for positive and negative burrs. There are four stages of burr development; initialization 
which is the primary shear zone, initial development, bending which is the pivoting point, and full burr formation. Continuous cutting consistently yields the lowest occurrence of burr formation, due to sufficient shear force to facilitate material removal. In ductile materials, cracks will initiate, grow, and produce a positive burr; in contrast, brittle materials, a crack will initiate, grow, and breakout to form a negative burr. See Figure 1.2 for burr development.

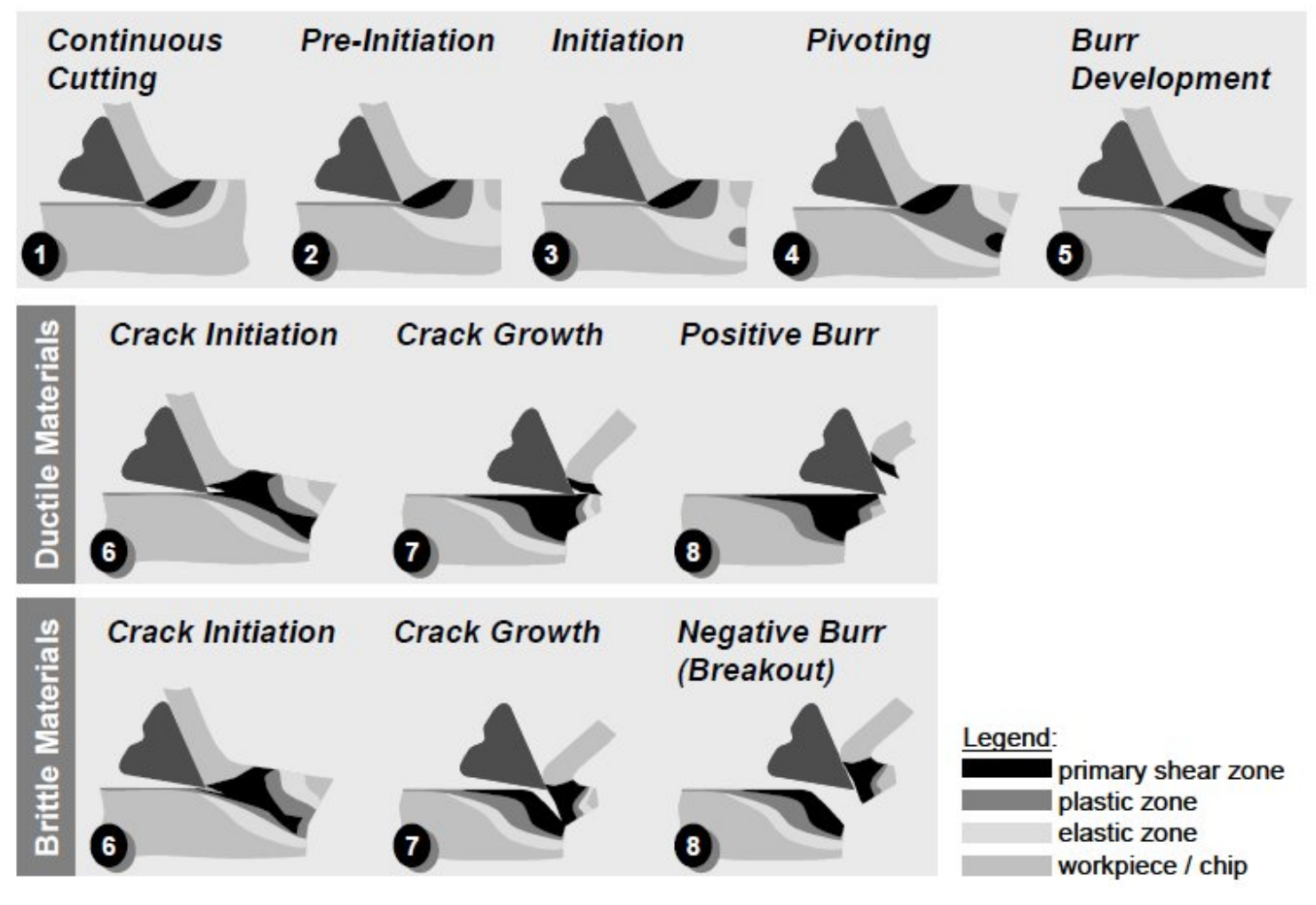

Figure 1.2 - Burr Development [2]

Burrs can be classified into four types according to Gillespie and Blotter [3]. Roll-over burrs form when the left over chip remains attached to the edge of the workpiece. Cut-off burrs occur when the chip at the edge of the workpiece rips off with more material than necessary to deburr. A Poisson burr is formed when the tool pushes into the workpiece plastically displacing material around the point on tool contact similar to a crater or wake. Tear burr forms when a chip tears more material than intended during material removal. See Figure 1.3 for four burr types. Many of 
the above listed burrs can be categorized into further sub categories, which is not in the scope of this thesis.
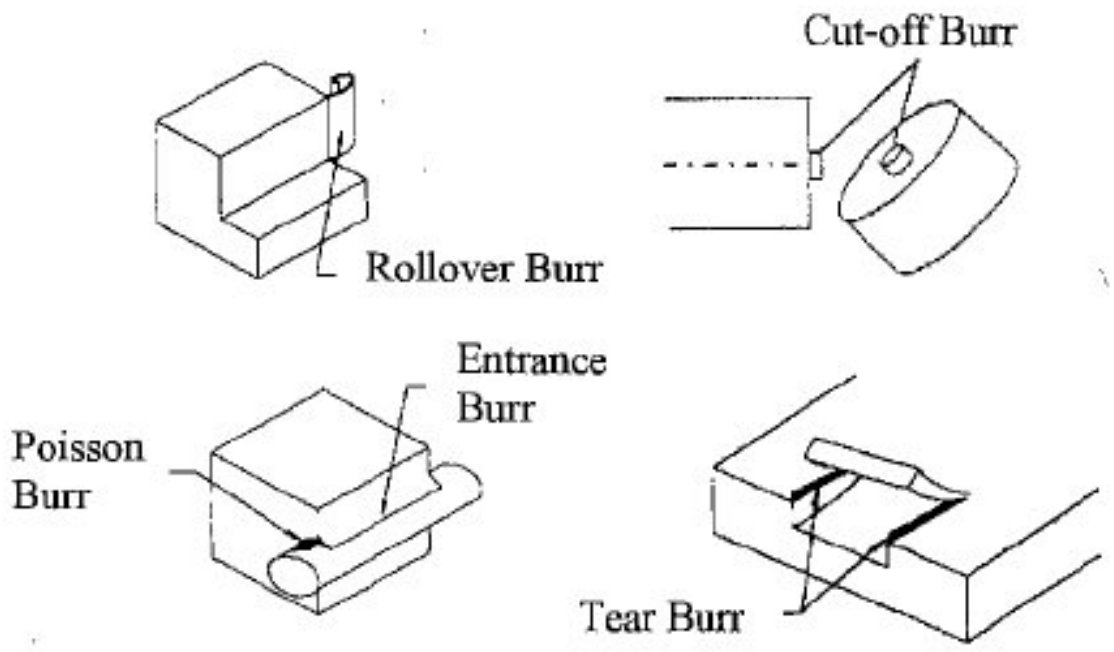

Figure 1.3 - Four Main Burr Types [2]

Burr geometry varies and is mainly classified by height and thickness. Kennedy [4] discussed that Weiler Corp. Cresco, PA had developed a classification system separating burrs into five classifications. Class one burrs are micro burrs that would occur in grinding operations. A magnifying glass would be required to see these burrs. Class two burrs break from the parent material fairly easily, usually with the use of soft media. Class three burrs are well-attached and require a coated abrasive for removal. Class four burrs are a harder attached burr and require moderate grinding; gas turbine engine parts predominately consist of this class. Class five burrs are major outcrops and will take significant grinding to remove. This classification system focuses on the type of method used for removal.

\subsection{Problem Statement}

This problem requires an automated process to use the principle of compliance to actively remove burrs formed on a workpiece feature. The automated process should use a hybrid tool to be a drop in replacement on devices that currently are capable of outlining the workpiece 
geometry. The automated process should provide sufficient results for tolerances of precision deburring requirements. The tool must use active compliance; during burr removal, the actuator must control the compliance, and the tool must sense the compliance requirement. By doing so this creates a hybrid active compliant solution.

\subsection{Outline of the Thesis}

Chapter two, a literature survey will describe the burr removal process. Compliance will be introduced and its importance during burr removal will be discussed. Existing methods for deburring will be summarized from research and from commercial application. Each will be evaluated for precision deburring of turbine engine components. Contact mechanics for abrasive wear will be outlined for its application to deburring theory. Methods of actuation for deburring will be evaluated and summarized.

Chapter three, the requirements parameters will be defined and stated. Design concepts will be presented and a final design concept will be chosen. The detail design of the radial actuator will be discussed.

Chapter four, the tool dynamics will be modelled in terms of stiffness and cutting force. The cutting force will be developed in terms of contact mechanics. The tool actuator interaction will be modelled and simulated.

Chapter five, the fabrication and assembly of the enhanced radial actuator will be explained and discussed. The tool's $\mathrm{x}$ and $\mathrm{y}$ sensor calibration will be explained and a relationship between the sensor output and displacement of the tool shaft will be determined. The tool's stiffness behaviour will be tested and compared to the simulation data. This data will then be compared to previous radial actuator stiffness behaviour. 
Chapter six, the results of the enhanced radial actuator will be discussed. The main research contributions will be outlined and summarized. Future work will be suggested for further research. 


\section{Chapter 2}

\section{Literature Survey}

The literature survey will outline current hand deburring techniques, processes and their limitations. The principles of compliance in deburring are outlined with various examples in existing research. The development of automated burr removal will be chronicled and evaluated for important changes that have been made throughout history. Actuation systems will be detailed and critiqued for their influence on precision deburring techniques.

\subsection{Burr Removal}

Deburring is the process of removing burrs and bringing the geometry of the part into acceptable tolerances. Burrs are removed today using hand deburring via skilled operators. Hand deburring is a labor intensive job and often provides less consistent results due to human error. It is the continued approach to deburr because it is versatile and takes up minimal floor space. It also requires less capital investment and may be the least expensive and efficient method to burr removal depending on the case. Hand deburring is also employed to remove burrs that are hard to reach, it is used if only a small number of parts require deburring, it is used to prevent media impregnation or oxide formation. When the part material properties make it difficult to hand deburr, a high production rate causes the operation to become a bottle neck in production; these are the contributing factors that yield the need for an automated process.

In manufacturing the flow of parts is important to gauge and measure the efficiency of the process used. When parts are machined on a NC lathe or mill and then transferred to an operator to manually remove the burrs this is a loss of efficiency as the mill can run continuously with very little down time and the operator cannot. In addition, the operator can be exposed to harmful grinding dust, and possibly repetitive-motion injury. 


\subsection{Principles of Compliance}

Active compliance control can be obtained through the actuation and feedback of position or force of the end-effector in a deburring solution. Previous work to create a compliant system has been either to monitor the force or position via multipoint contact or an end-effector with position or force feedback integrated into the end-effector.

Compliant tooling is a way of reducing chatter and tool path planning or programming time. A compliant tool yields to the part's irregularities allowing programmers to spend less time creating a tool path plan. Force control may be required for greater variations in burr size. There are two basic approaches; through the arm control and around the arm control. In through the arm control, a force sensor sends feedback to the robot controller, which dynamically adjusts the toolpath. Around the arm control utilizes a device between the robot and the tool that operates independently and maintains a programmed force by the tool on the workpiece [4].

A passive compliant tool in its simplest form is intentionally deformable. There has been much research into novel devices that provide a more complex level of compliance. In Schimmels [5] [6] research a jig or frame was created to give compliance to a robotic endeffector. The jig provides a unique position and orientation in the plane orthogonal to the workpiece edge while also allowing motion along the edge. The jig is given stiffness through torsional springs that provide a low degree of specified directional coupling. The device seeks to align itself with features on the workpiece and resists loss of alignment despite error in the commanded position and orientation. The jig is best suited to ensure that the unique relative positioning is initially attained and maintained, despite small finite positional errors. It is not suited for larger finite positional errors; it is limited to its frame size which is currently fixed. This would be an issue for workpieces with complex and small features like that of turbine engine components. 
Liao, Xi, and Liu [7] have developed and tested an integrated tool head that has force control. The tool used a pressure controlled pneumatic cylinder to create variable compliance with the workpiece, through the normal contact force. The tangential contact force or friction of the tool was regulated via the variable torque of the tool spindle. Experimental results for the tool had shown the control scheme developed could control the tool head.

In Kramer and Shim's [8] work on force controlled compliance, a deburring process for a passive compliant end-effector was demonstrated using an AdeptOne semi-direct drive, four-axis SCARA configurable robotic system. To drive their remote center of compliance they were able to instrument deburring for two axes of force on the x-y plane and on z-axis with torque. A closed loop control system was used create a constant normal force for burrs of modest size. Testing was completed on a workpiece made from aluminum 6061. This edge tracking and force regulation process yielded acceptable results. This method would not work for a large variation in burr sizes as well as has not been tested for harder metals like those used in jet turbine engines.

\subsection{Existing Methods for Automated Deburring}

Methods for compliant burr removal have been studied and many different systems have been designed and tested. Active and passive compliant systems have been developed for burr removal. Passive compliant systems offer a set compliance value through the stiffness of the system. Active compliant systems offer a dynamic compliance value through either the changing of stiffness of the system or the displacement of the tool head to compensate for the lack of stiffness required.

Her and Kazerooni [9] developed a method for control of the metal removal process and tracking of the workpiece surface. They designed a tracking mechanism to measure force of the robot endpoint. The normal cutting force was calculated via a force sensor to create a stable 
metal removal process. The tracking can be used on a workpiece with unknown geometry ensuring that the tool tip always remains in contact. The authors were able to maintain a relatively constant cutting force in the tangential direction by varying the tool velocity along the part edge. The experiment was done using a very stiff, stable, and precise end-effector; an x-y actuated table. This experiment would be more difficult for a robot mounted system, as the position and uncertainty of the end-effector would require the need for another force sensor, and roller bearing mechanism. This mechanism only facilitates deburring of surfaces with a smooth enough transition that the bearing will pass over the surface without meeting any discontinuities. Application of this method to turbine engine components is not sufficient to complete the task of deburring.

ATI have created several passive compliant deburring tools for radial and axial deburring, as well as an axial compliant finishing tool. In Figure 2.1 the two black tools located on the left side, are the ATI axial deburring tools Speedeburr. These tools provide passive compliance in the axial direction via a floating rotary cutter, which provides a constant cutting force. This tool was exclusively designed for edge deburring and chamfering. In Figure 2.1 the upper three deburring tools to the right and the lower left tool are ATI's Flexdeburr tool. These tools are passively radial compliant and use a series of pneumatically actuated cylinders pointed at shaft of the tool. The shaft of the tool is also fixed to a gimbal which allows for movement of the shaft in only the radial direction. 


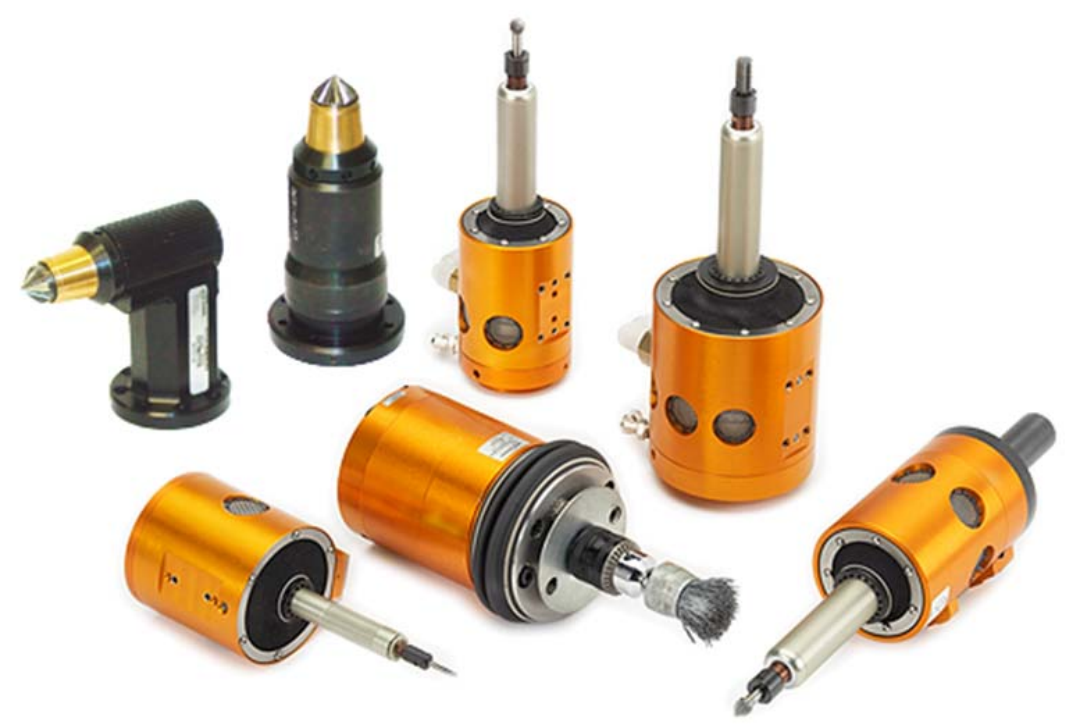

Figure 2.1 - ATI's Deburring tool family [10]

In Figure 2.1 the remaining tool in the lower center is the VersaFinish, a primarily polishing and finishing tool with passive axial compliance. The tool performs in the same manner as the Speedeburr tool, however has a chuck for holding customer-supplied tools. This tool also has the ability to be equipped with an optional sensor for detecting contact of the media with the workpiece. ATI also provides multi-axis force and torque sensors that when paired with the deburring tools can achieve active force compliance. This option is however a separate unit and is not an out of the box solution to active force compliance. ATI's deburring tools are for light to medium duty materials such as aluminum, plastic, and steel. These tools are not designed to handle materials such as Waspaloy, Inconel or other high nickel super alloys commonly used in turbine engine components. ATI deburring tools are not designed for tolerances of turbine engine components.

At Ryerson University Liao [11] developed an active force compliant deburring tool. The tool consisted of a set of Festo pneumatic cylinders mounted to a tripod robot with an extensometer for positional sensing of the tool tip, see Figure 2.2 below. This tool was only 
axially compliant as it only had compliant actuation in one direction, the tripod robot provided tool path actuation only. Air supply to the Festo cylinders as well as the pneumatic spindle provided control over the cutting force and contact area.

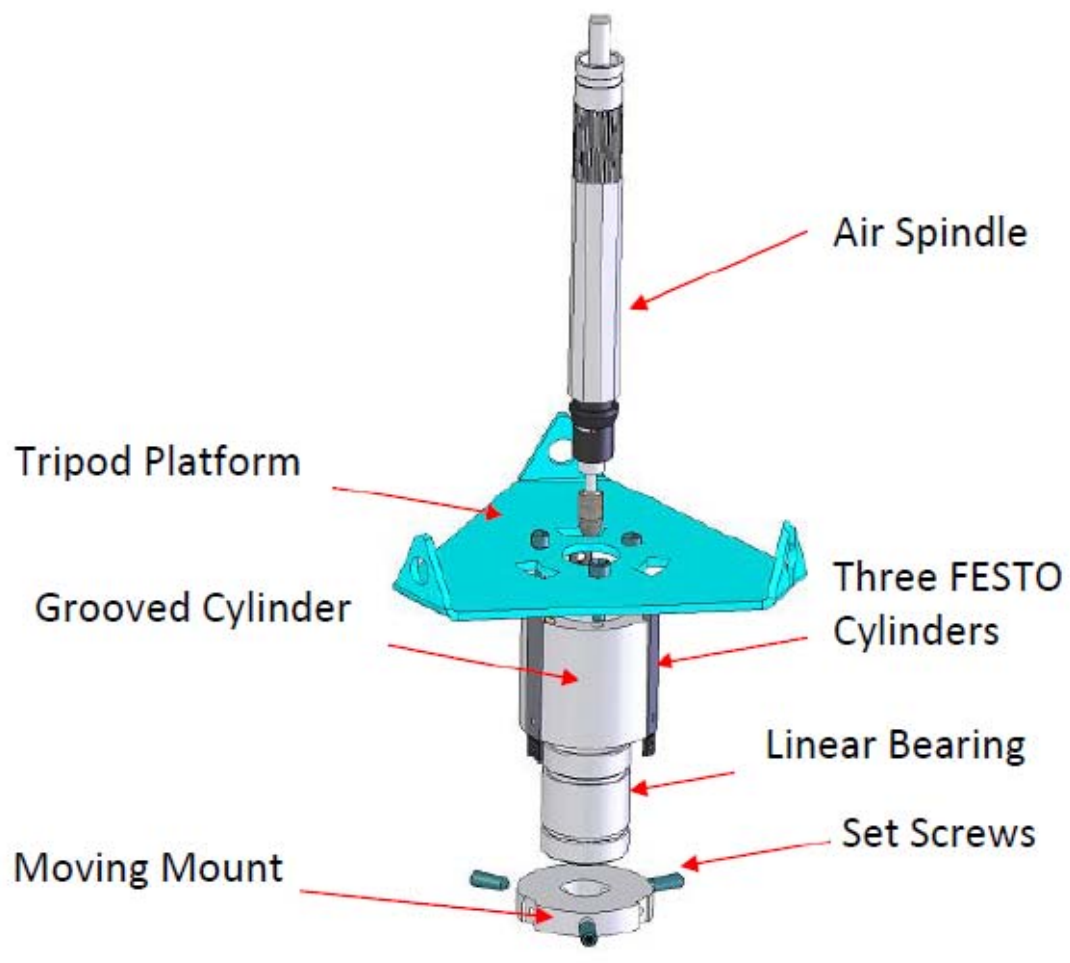

Figure 2.2 - Axial Compliant Toolhead [11]

At Ryerson University Petz [12] in his work on designing of a radial-axial hybrid force compliant tool head was able to develop a radial-axial hybrid force compliant deburring tool designed to handle burrs on turbine disc components. Building off of Liao's [11] design for the active axial compliant toolhead his design incorporated active radial compliance. As Liao had done the axial compliance was actuated via pneumatic cylinders, see Figure 2.3 below. 


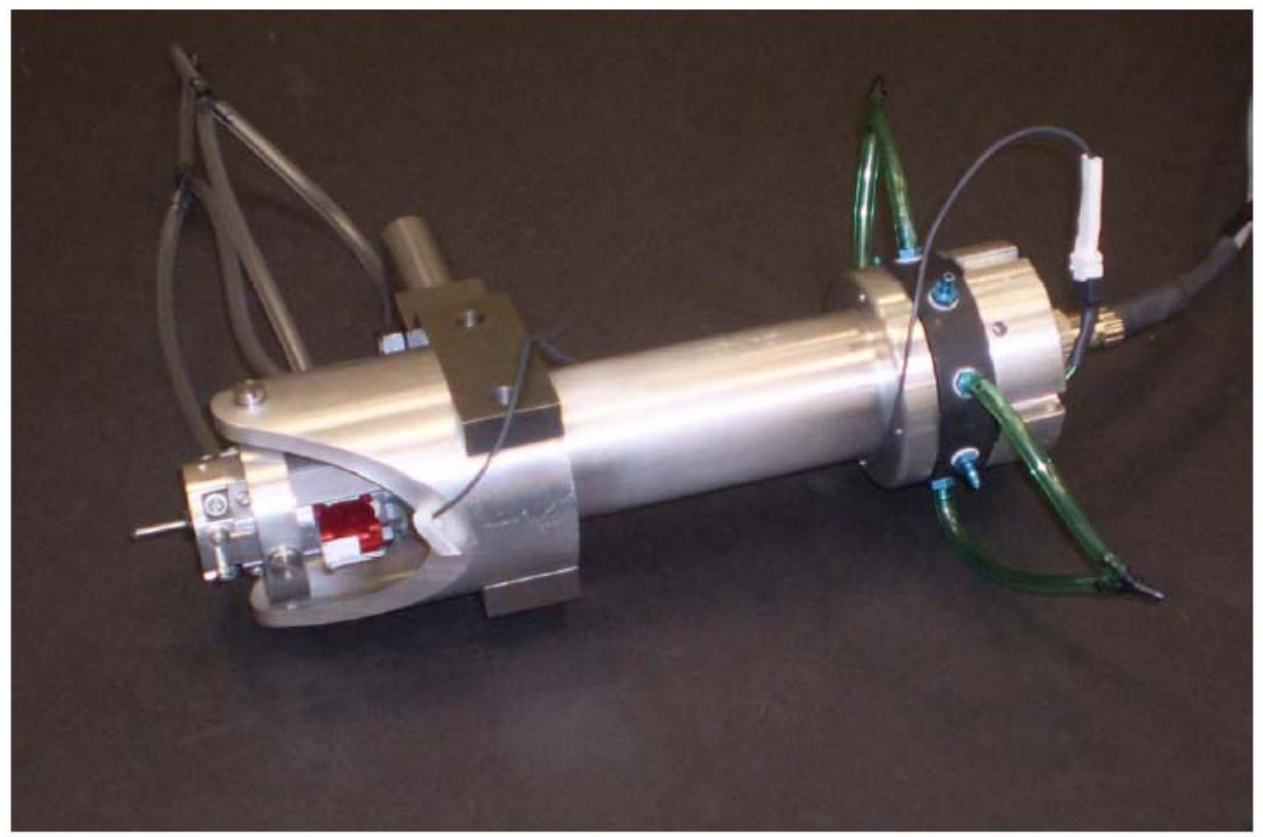

Figure 2.3 - Radial-axial hybrid active force compliant deburring tool head (HFCDT) [12] In contrast to Liao's [11] linear encoder, a string pot resistor was used to signal the axial position. The addition of the string pot improved the performance and reliability of the axial position. Active radial compliance was achieved using the pneumatic ring actuator (PRA). The PRA was constrained in the outer radial and in both axial directions. Inflation would then force displacement into the centre of the PRA where the shaft of the tool sits. A gimbal on the opposite side of the tool allowed the shaft to rotate in two degrees giving it radial displacement. A magnetic field sensor sat above the assembly tracking magnets on the tool shaft. As the tool rotates the sensor tracks the radial displacement of the tool. As the tool displaces the PRA was able to inflate and increase pressure towards the centre, forcing the shaft to the centre of the tool. Displacements caused by burrs could be detected, and the pressure of the actuator increased and decreased to remove the burr.

\subsection{Actuation}

Throughout research in the past 30 years many automated deburring techniques have been proposed. Solutions have been proposed to achieve the deburring process with active 
compliance. There are typically two types of actuation possible electric and pneumatic.

Hydraulic fluid pressure is typically not used as the system response is typically low, the size of a hydraulic system would be too large, and the forces involved exceed deburring size and force requirements. Mechanical actuation is usually used in conjunction with one of either pneumatic or electric sources.

Electric actuators are usually in the form of a servo motor. A servo motor is a rotary actuator used to control angular position with a feedback sensor for position. The position sensor is usually an encoder or potentiometer. Most automated deburring techniques in research have used this method, as the robot arm or end-effector is often used to actuate the force of the tool tip on the workpiece. However, this method is a modification of the system to which is solely used to deliver toolpath position control. It is considered a decoupled tool if the toolpath control is not affected by force. Stouffer et al. [13] discusses the Adaptive Deburring Tool (ADT) developed by TriKinetics Inc. uses two servomotors with worm screws to actuate the radial position of the tool, see Figure 2.4 for a diagram of the tool.

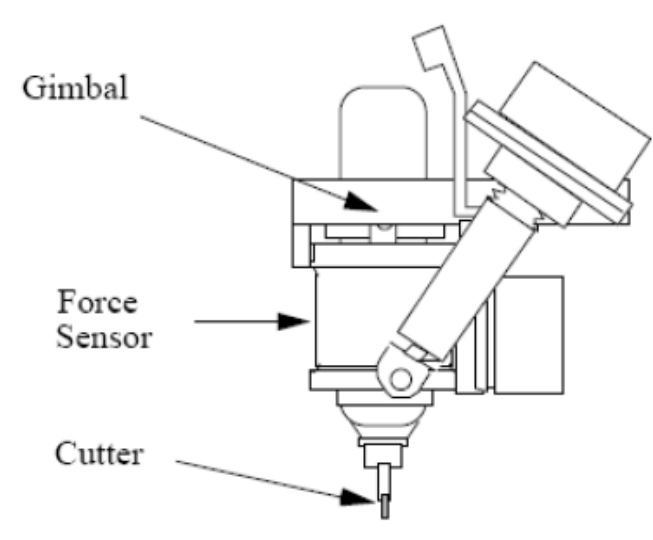

Side View

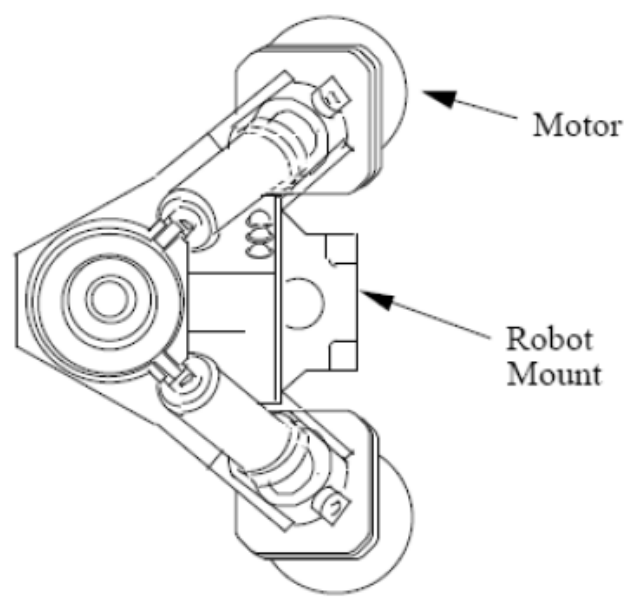

Bottom View

Figure 2.4 - TriKinetics Adaptive Deburring Tool [13] 
The two actuators work in conjunction with a gimbal that allows for the pivoting motion required for radial actuation in two degrees. The actuator was deemed to have a reaction time too slow for precision deburring.

Stouffer et al. [14] discusses the United Technologies Research Center (UTRC) developed the Chamfering and Deburring End-of-arm Tool (CADET) to provide active force compliant deburring, see Figure 2.5 for a diagram of the tool. CADET used two gimbals in its design. The first gimbal was used to actuate force sensors which detected radial forces on the tool. The second gimbal was used as a pivot and allowed four linear voice coil actuators connected at the top of the gimbal by a spider or universal joint to create active compliance. The linear voice coil actuators perform as speakers do where a voice coil charged with electricity provides a magnetic force that when paired with a permanent magnetic collar can act as an actuator. 


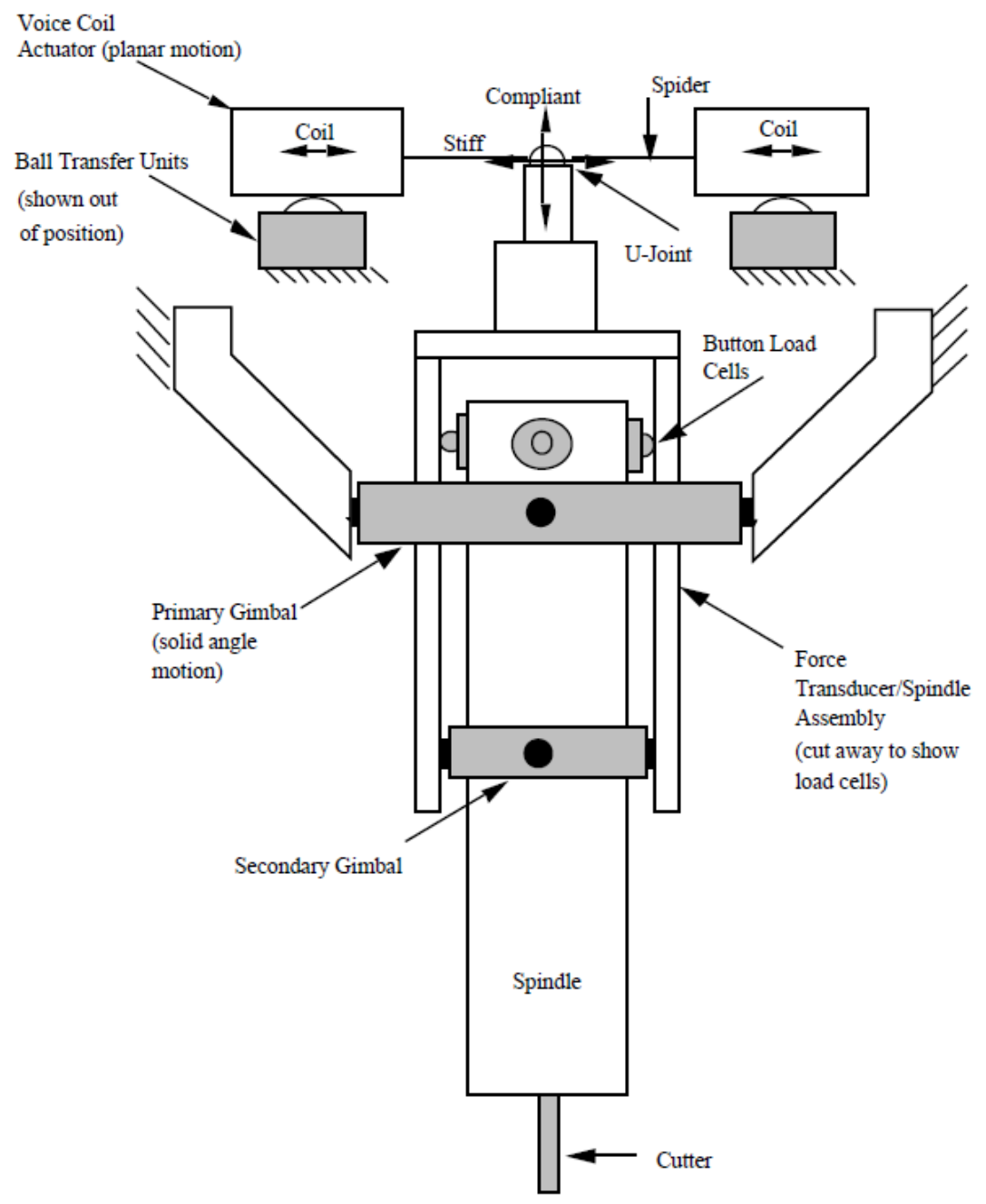

Figure 2.5 - United Technologies Research Center (UTRC) Chamfer and Deburring End-of-Arm Tool (CADET) [14]

Pneumatic actuators are more commonly used for actuating active compliant deburring. A pneumatic system converts the energy from compressed air into mechanical motion. This conversion is usually in the form of a pneumatic cylinder. Often this is accompanied by a spring whose pressure must overcome in order to actuate. This ensures that the actuator moves to fill the space to which pressure has reduced causing a proportional cylinder volume to pressure relationship. Commercially active compliant deburring exists for axial deburring only. Speedburr by ATI accomplishes this through the pneumatic actuation of its deburring tool tip. The tool tip 
acts as a piston in this fashion. Similar work has been done by Liao [11] using a group of Festo pneumatic cylinders to actuate the tool tip. See Figure 2.6 below for an image of the compliant tool head.

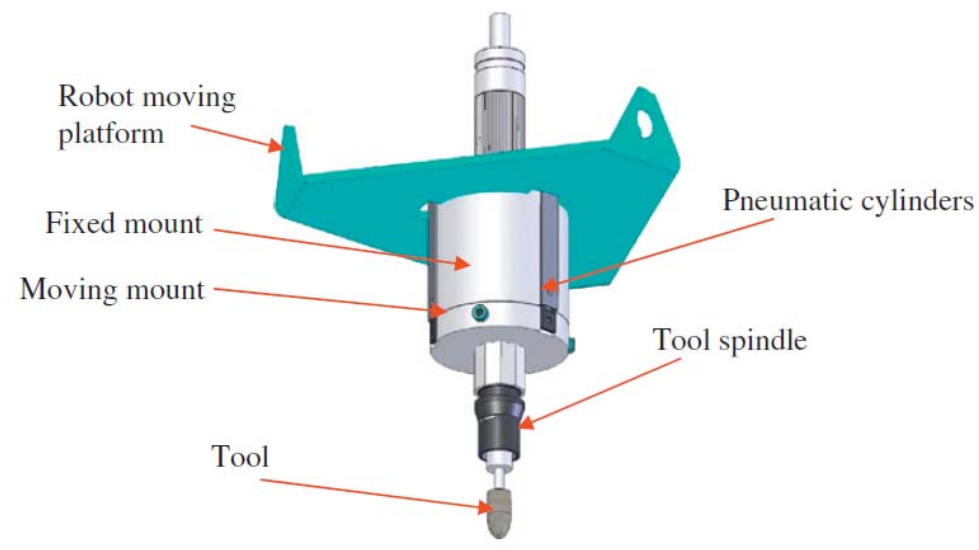

Figure 2.6 - Axial Compliant tool head [7]

The force of each pneumatic cylinder is due to the simple relationship between the pressure in the cylinder and the surface area of the piston. The force of the piston over the stiffness of the spring is equal to the displacement of the piston.

Chung and Kim [15] in their work on a dual action deburring tool developed a deburring tool that achieves deburring through a first and second pass deburring tip with a single toolpath. A similar model was used to describe their deburring process as Liao [11] had used for the axial compliant deburring tool. The model was different in that it does not rely on a spring stiffness to counter balance the air pressure; instead it uses the dynamic pressure between the top and bottom of the piston. The lower chamber is used to retract the tool tip and the upper chamber is used to force the tool tip out. The system was modelled using a mass flow approach dependent on the pressure entering the chamber and its volume. ATI has also incorporated a similar pneumatic cylinder system into a radially compliant tool. The Flexdeburr tool has an array of radially positioned pneumatic cylinders, much like previous pneumatic designs, see Figure 2.7 below. 


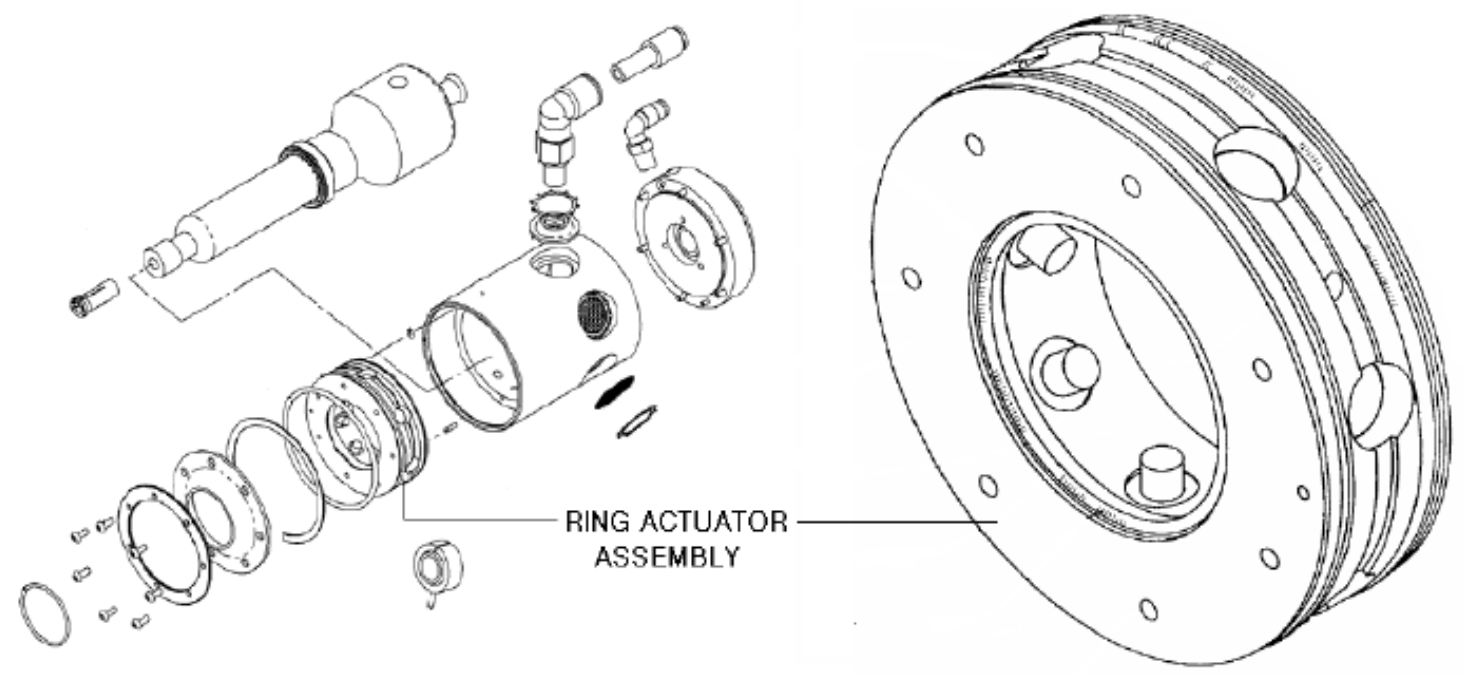

Figure 2.7 - ATI ring actuator assembly on the Flexdeburr tool [10]

It is apparent that the cylinders rest on the outer diameter of the tool shaft. The tool shaft was mounted to a gimbal; the tool's passive compliance is determined by a set point pressure in the cylinders. The displacement of the tool was not sensible and does not influence the pressure in the cylinders. The pressure in each cylinder should be the same as the compliant pressure.

Petz [12] in his work on a radial-axial active compliant deburring tool used a similar design to Liao [11] for the axial actuator deburring component of the tool. Four Festo pneumatic cylinders were used to create axial compliance. Each actuator was modelled as a spring and damper with associated stiffness and damping components. The radial actuator was called a pneumatic ring actuator (PRA) and functioned as a rubber bladder or diaphragm configured in a torus shape, see Figure 2.8 below. 


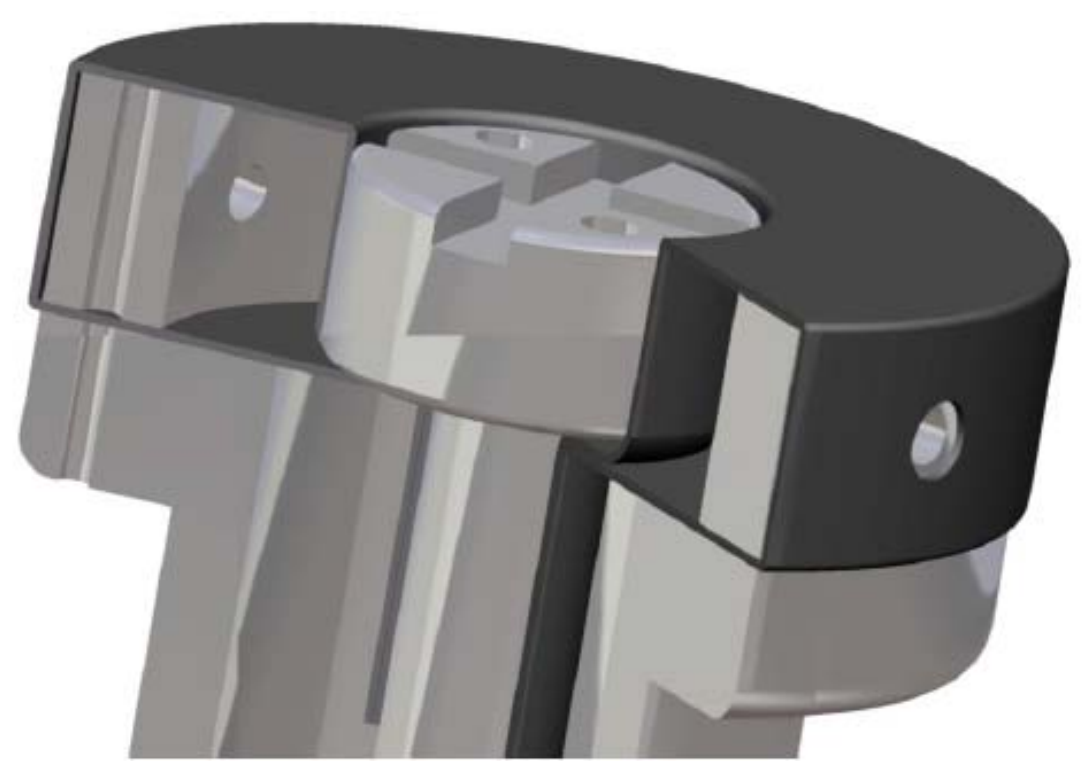

Figure 2.8 - PRA bicycle inner tube stretched around a conduit ring [12] A bicycle inner tube was fashioned into a torus and used to actuate the tool shaft to a centred position actively. Petz [12] concluded that this design could be improved as it did not fully demonstrate the results that were being sought out. Due to the design, manufacturing the intended shape was difficult and inconsistent. Performance of the PRA also seemed to be nonuniform which made defining its performance difficult.

Rubber diaphragm actuators have been used by Grosjean et al. [16] in work on micro balloon actuators for aerodynamic control. The research was focused on using an inflated diaphragm as an actuator on the leading edge of a transonic aircraft for aerodynamic control. An array of rubber diaphragms were fixed in a window fashion and inflated into the path of airflow over the wing. In the article, silicone rubber was used and deposited to the surface of a material, a method developed by Yang et al. [17]. Both an adhesive layer and release layers were deposited to create pockets. The silicone rubber used was MRTV-1 with a Shore A hardness of 24; this is a very soft rubber. 
Electric and pneumatic actuators have three trade-off considerations. The response time of the actuator will be different. Electric actuators will typically have a faster response time; compared to pneumatic actuators tend to be sluggish. The power sources of the actuators are

different. Electric actuators require a higher voltage consumption power source to be fed right to the end-effector of a robot arm or $\mathrm{CNC}$ machine. Pneumatic actuators require an air hose of compressed air which is the common and readily available source of power used today. Pneumatic actuators tend to have an inherent compliance characteristic whereas electric actuators require constant active manipulation control.

\subsection{Summary}

This literature survey discussed the short falls of the current hand deburring practice. Solutions to deburring so far have not accomplished this for precision deburring in both axial and radial directions. Regardless many characteristics of these solutions can be drawn on for their strengths. Actuation and sensing will continue to be improved and drawn upon. ATI's Speedeburr and Liao's [11] axial compliant deburring actuation will continue to influence the next generation of axial compliant deburring. ATI's Flexdeburr, Petz's [12] radial actuated deburring tool will continue to influence the future of radial compliant deburring tools. A solution to the problem of automated precision deburring must consider past research and products available on the current market. Compliance can give a deburring tool the ability to adapt to inconsistencies in both the burr size and part position. 


\section{Chapter 3}

\section{Tool Design}

Previously it was discussed that hand deburring methods for precision deburring have left the requirement for an automated process to be developed. An automated precision deburring process must possess compliance when contacting the workpiece. The deburring of turbine disc parts has been explored by previous work. None of the solutions presented have provided a solution that meets compliance requirements needed for precision deburring. In the following chapter the requirements and design parameters will be discussed with the focus on compliance of the deburring tool. Possible design ideas will be presented and their strengths and weaknesses will be weighed for their merits to arrive at a final design concept. The detailed design of the final solution will be described in terms of its actuation and sensing capabilities.

\subsection{Requirements and Design Parameters}

A deburring tool must be approached first by the principle of its design, compliance between tool tip and the workpiece. Petz [12] has suggested the simplification of the solution through the use of a rotating action plane. The rotating action plane allows for the $\mathrm{x}$ and $\mathrm{y}$ axis to become one axis, the radial direction and the $\mathrm{z}$ axis the axial direction. The action plane will rotate about the $\mathrm{z}$ axis. This assumption can be made if the stiffness relationship is uniform through the $\mathrm{x}-\mathrm{y}$ plane. See Figure 3.1 below for a definition of the action plane. 


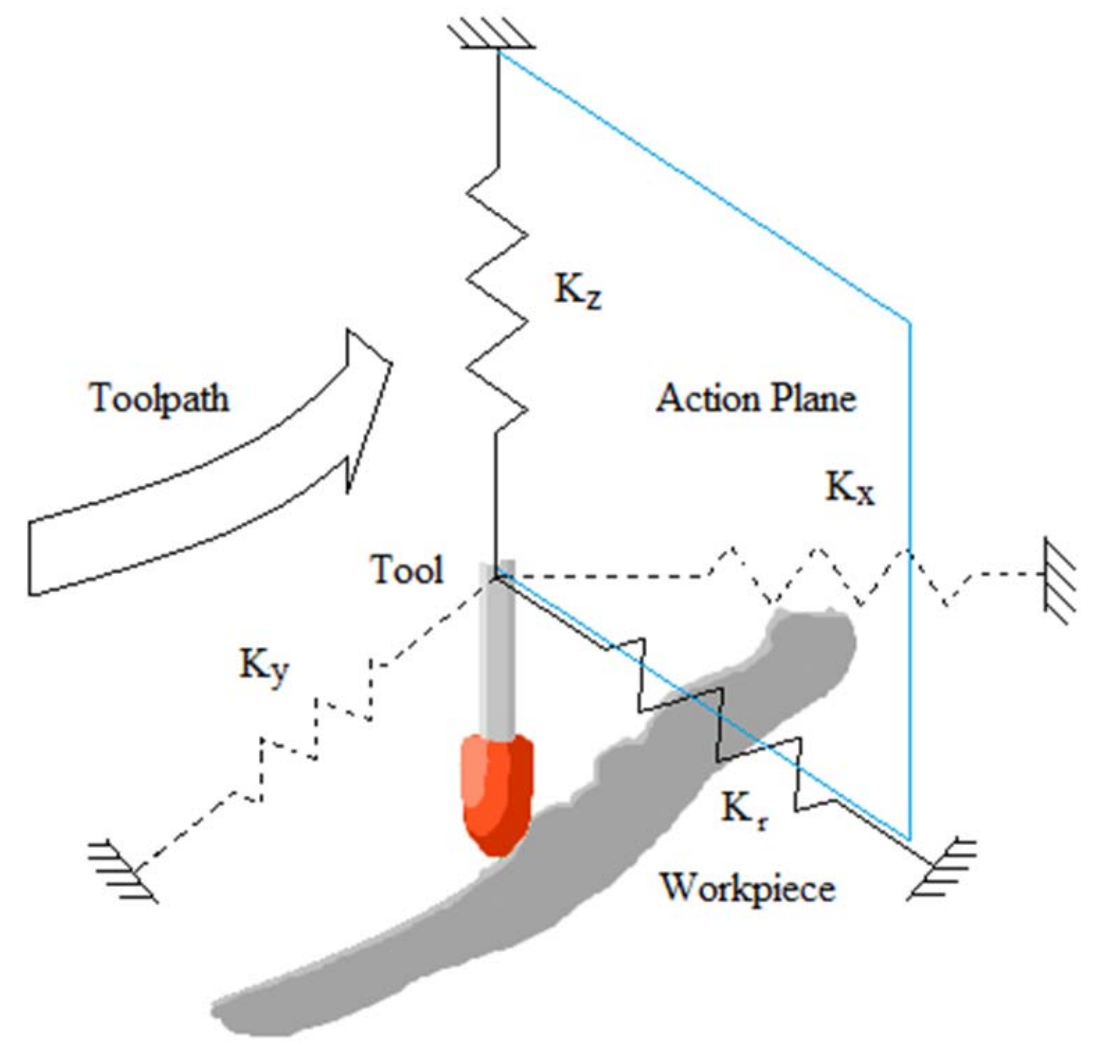

Figure 3.1 - Definition of the action plane

In all Cartesian degrees of freedom, the tool tip contacts the workpiece and a reaction force exists due to the cutting interaction. The cutting force continuously changes throughout the deburring process; in turn active compliance is achieved by balancing this force with the tool's stiffness. This was modelled by Liao [11] for axial compliance. Axial and radial actuation is needed due to the angle of trajectory of the tool tip, when dealing with complex workpiece topology. Figure 3.2 illustrates the tool tip trajectory with the resultant contact force in green. The radial axis is denoted by $r$ while the axial axis is denoted by $z$. 


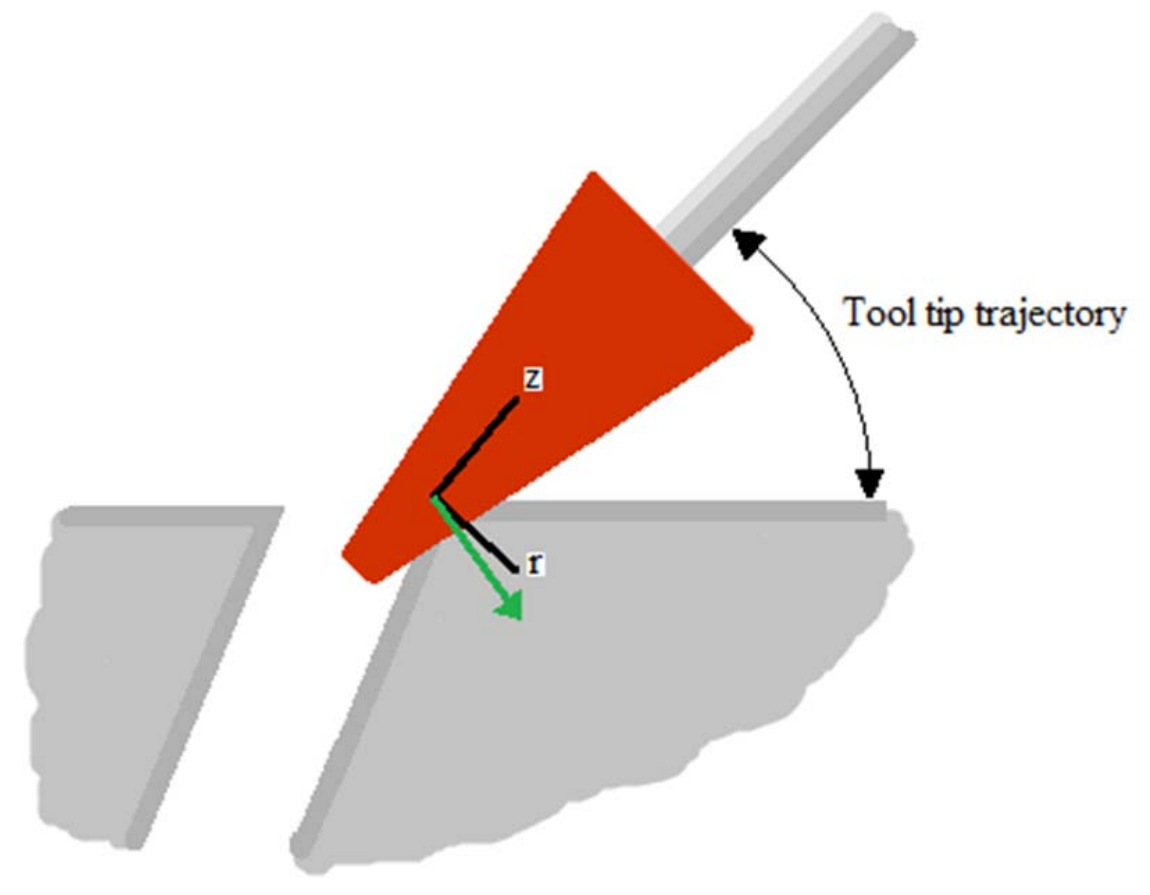

Figure 3.2 - Tool Tip Trajectory

The design of the tool must be actively compliant in both axial and radial directions. This would give the tool three degrees of freedom to allow for the most robust burr removal possible. Integrating these two systems is a difficult task as they must be decoupled from the feed of the tool path. A gimbal has been used in Petz's [12] HFCDT, ATI's Flexdeburr, TriKinetic's ADT, and UTRC's CADET to achieve simplified actuation in at least a radial direction. This is a requirement due to the universality of its design and function. See Figure 3.3 below for the system model with the use of an action plane. 


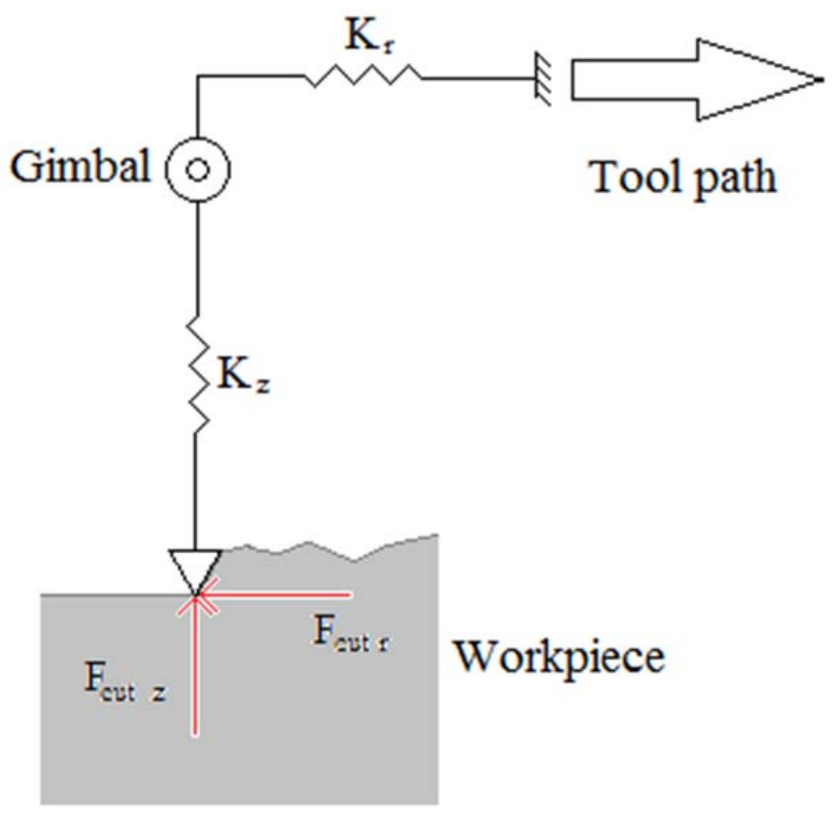

Figure 3.3 - Deburring tool model within action plane

The action plane along with the gimbal guarantees compliance in both radial and axial directions. The tool deflects in the radial direction due to the tool stiffness in the radial direction being lower than required for a constant contact force or feed rate along the tool path. This will result in an increase in radial tool stiffness. The increase in radial tool stiffness will cause the tool to remove the excess burr and continue along the tool path. This process will occur in the same manner for a burr in the axial direction as well.

Now that the definition of compliance for the tool has been established, it is important to establish a metric to define the tool's ability for compliance. Compliance is the ability of the tool to maintain contact and cutting force with the workpiece. When the tool encounters a burr or an inconsistency in part dimension or position this compromises the tool's compliance with the workpiece. The natural range of compliance can therefore be defined by the burr size and the force required for removing them in deburring turbine disc parts. Roberts et al. [1] have defined typical break edge depth to be $0.1-0.4 \mathrm{~mm}$. See Figure 3.4 for a diagram for part edge finishing. 
According to Petz [12], the resolution for sensing displacement should be less than $0.1 \mathrm{~mm}$, the minimum break edge depth. Roberts et al. [1] have also defined a range of contact force required for edge finishing turbine disc parts of 0-20N.

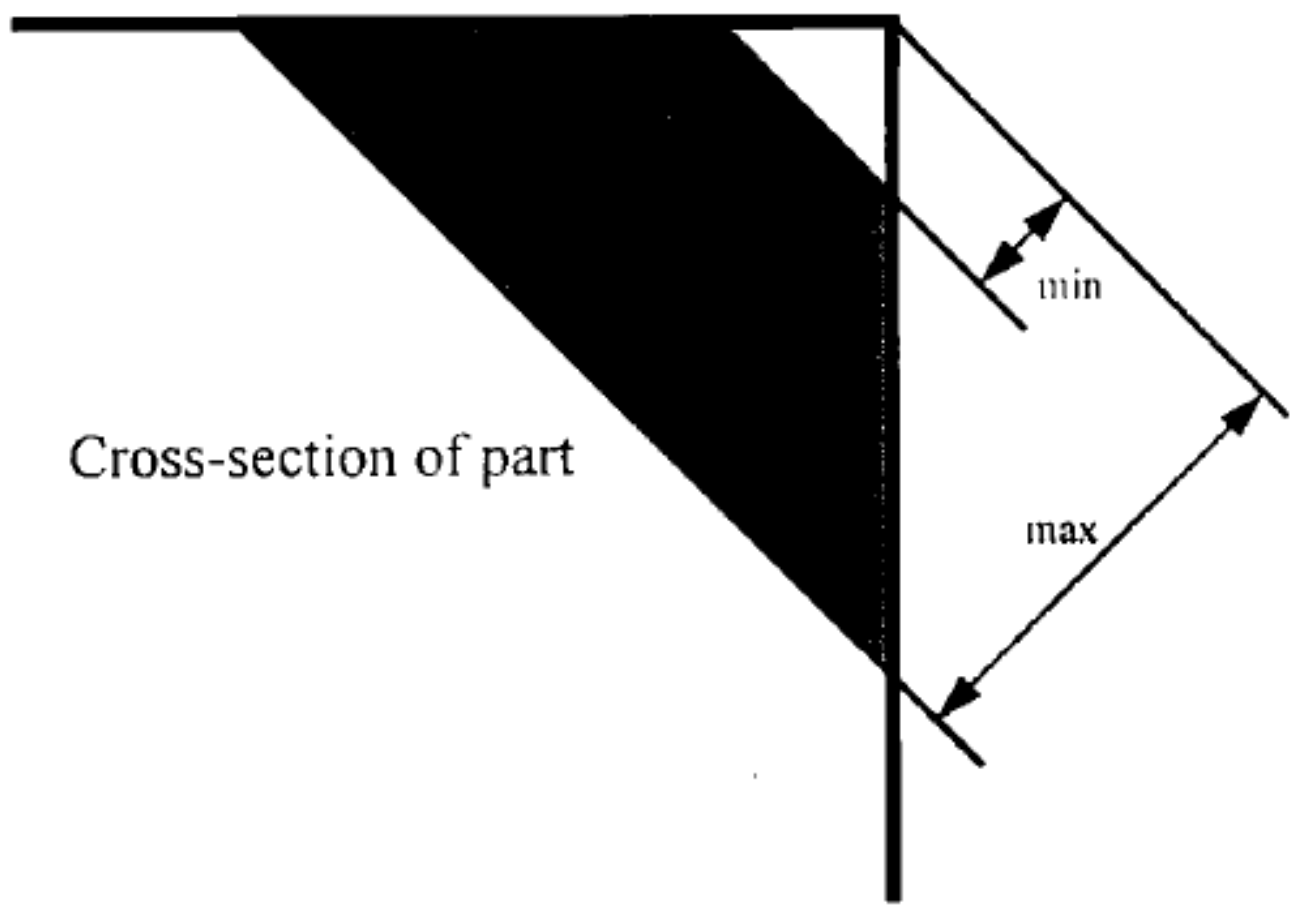

Figure 3.4 - Maximum and minimum for edge finishing [18]

Compliance is characterized as the inverse of stiffness. This would mean that the stiffness of the tool is important in determining the tool's ability to remove a burr. A relationship between the input parameters and the stiffness is important in determining the tool's compliance characteristics. According to Roberts et al. [1] the stiffness required for precision deburring and chamfering operations is in the range of $10000-30000 \mathrm{~N} / \mathrm{m}$. Although this depends on cutting force, feed rate, spindle speed, and tool wear this range will be a design point.

Now that the primary design considerations have been determined, the secondary design considerations should be explored. The tool will be mounted on a robotic arm or CNC mill; it could be used in conjunction with a gantry or indexing table. This would provide the feed for the 
tool only. The tool must be light enough to be mounted to a robot or CNC machine. The tool's internal components must be rigid, with the exception of any non-rigid flexible designed components. Sensing is required in both axial and radial directions; this can be achieved by a single sensor or multiple sensors to determine axial and radial location of the tool.

The tool should be designed for optimal manufacturability. This not only affects the machining of components of the tool, but also the assembly and cost effectiveness of it. Manufacturability also includes assembly of the tool. Petz [12] describes that the PRA's design was difficult to assemble and that this had a dramatic effect on the reliability of performance the tool demonstrated.

The budget for development, fabrication, and testing materials was $\$ 2000$. The tool must be testable, as it must provide the ability for calibration and testing equipment to be mounted on it; furthermore size a large factor to consider. Considering resources available it is important to reflect on past work completed on this subject. The radial-axial hybrid force compliant tool head as developed by Petz is a good foundation for this research. However, the radial active compliant system required further development. As highlighted by Petz [12] the PRA should be further developed to provide a more consistent shape and a more conventional and convenient application. Due to radial-axial hybrid force compliant tool head being available for future modification and study it was considered that all components and hardware is available for reuse or repurposing. It must be considered that the weakest portion of the tool the radial actuator be considered for redesign while the strongest portion of the tool the axial actuator be reused. Understanding the dimensions of the previous tool is an integral part of the requirements for design of a new radial actuator.

The requirements and design parameters have been established via previous work. The summary of requirements: 
- Must redesign radial actuation of HFCDT

- Must fit onto existing HFCDT

- Must use an action plane for reduced tool complexity and increased efficiency

- Must provide at minimum compliance for a stiffness range of $10000-30000 \mathrm{~N} / \mathrm{m}$

- Must provide a range of $0-20 \mathrm{~N}$ of contact force at the tool tip for deburring

- Must be manufactured within a $\$ 2000$ budget

- Must have a tool tip displacement range of at least $0.1-0.4 \mathrm{~mm}$

- Must have a displacement resolution of less than $0.1 \mathrm{~mm}$

\subsection{Design Ideas}

The previous HFCDT has become the platform for an improved radial-axial active compliant precision deburring tool. The weakest component of the HFCDT is the radial actuation system. See Figure 3.5 below for an image of the PRA designed by Petz [12].
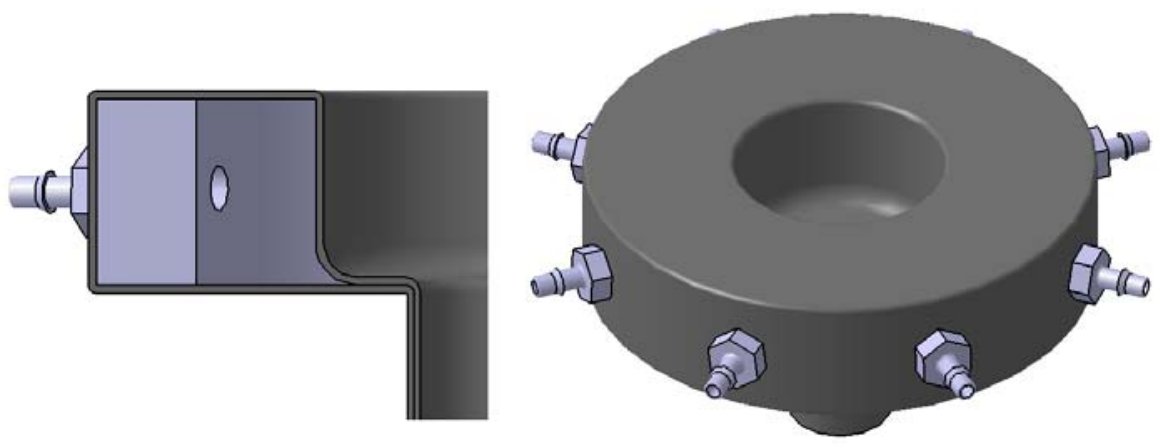

Figure 3.5 - HFCDT PRA cross -section and isometric view

The PRA depends on the underside of the sensor that sits above it, the top surface of the tool housing which it sits on, and the tool shaft that it surrounds and it actively brings to centered position. The lower portion of the rubber material is clamped to the shaft of the tool using sealant and a hose clamp. Through analysis of the assembly process it is apparent that a significant amount of preloaded tension was created in the rubber diaphragm of the PRA. Tension in the 
diaphragm led to irregular performance of the designed component. Inconsistent results could be attributed to the lack of symmetry during the assembly process.

The first design concept uses four block shaped pistons similar to the ATI Flexdeburr ring actuator. As can be seen in the cross-section in Figure 3.6 the main housing provides the four surfaces for each piston to slide on. An end cap on each cylinder for which a port is installed to supply air pressure. The pistons have a concave rounded shape to the end of them to cradle the tool shaft in an attempt to increase contact area. The material selected for this design is 6061 Aluminum as this is consistent with the rest of the tool. The manufacturing of this actuator would have been moderately difficult. The main housing is flat milled with exception of the four shafts. These are set on special tooling or an indexing mill. The pistons would be flat milled as well as the end caps. The end caps are fixed to the main housing using an adhesive, although this is not convenient for disassembly. The actuators could not be pressurized in the absence of the tool shaft. The tool shaft would keep the pistons from leaving the contact of the housing. The tolerance between the housing contact area and the pistons would be very important to the performance of the actuator. The tolerance would cause there to be too much interference. The piston would not slide freely enough in the housing as a result. Too large of a gap would interfere with the force achievable on the piston. The contact of two metals would require a lubricant to ensure properly performance of the actuator. This would introduce unwanted contaminates onto the workpiece or the rest of the deburring tool. This would require a collection system to contain any lubricants. When the tool shaft contacts the actuator at a 45 degree angle it would likely cause one or two pistons to become jammed. Instances of this would become more likely as the piston follows the tool shaft away from its housing. 

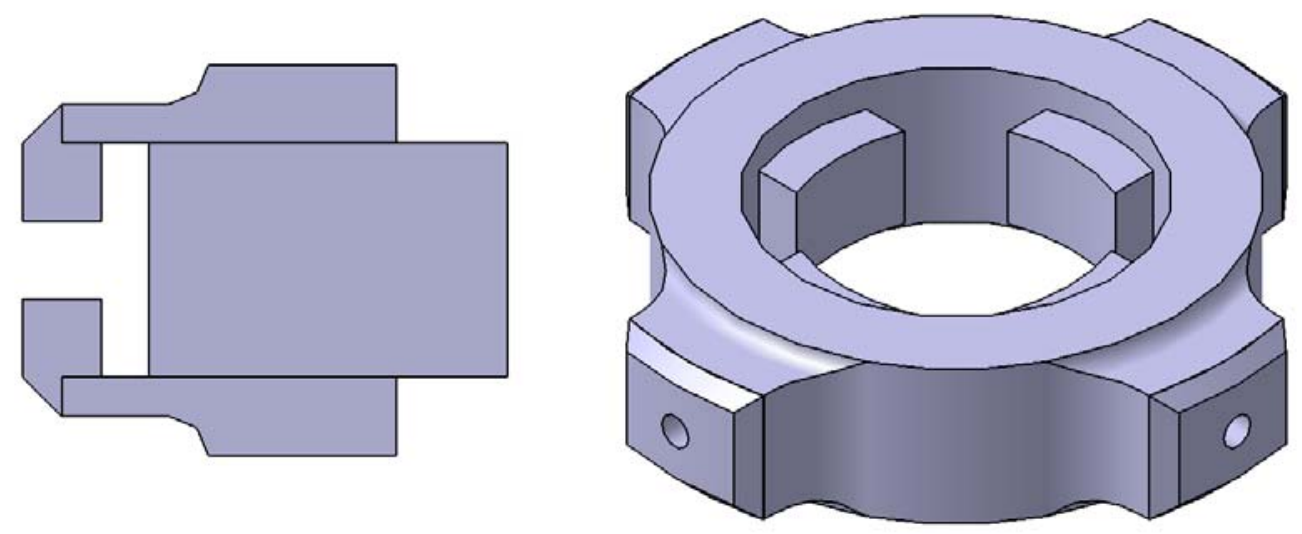

Figure 3.6 - Four Piston Actuator Concept

A second concept of the radial actuator was created to better address some of the limitations of the first concept. The design is under the same principle as the first concept. Actuation of the shaft was created by air pressure in a chamber behind the piston. There are multiple pistons to contact and act on the tool shaft. A significant improvement is the addition of a stepped piston, see Figure 3.7. The step in the piston allows it to be pressurized without the presence of the tool shaft. The step will stop the piston from projecting past the housing. This concept also addresses the first concept's issue with jamming. The increased number of pistons reduces the likelihood that jamming would occur, as a wider angle of contact coverage would be present. The end caps could be assembled using an adhesive as the previous design and would pose the same difficulties during disassembly.

This concept will be significantly less complex to manufacture. All of the components can be flat milled with no complex radii or surfaces. The simplification of this will reduce the cost significantly. The cost reduction would offset the increased number of parts required. As was with the first concept the tolerances of the piston and the housing openings must be such that they are not in interference or too large and therefore ineffective for controlling the tool's stiffness. 

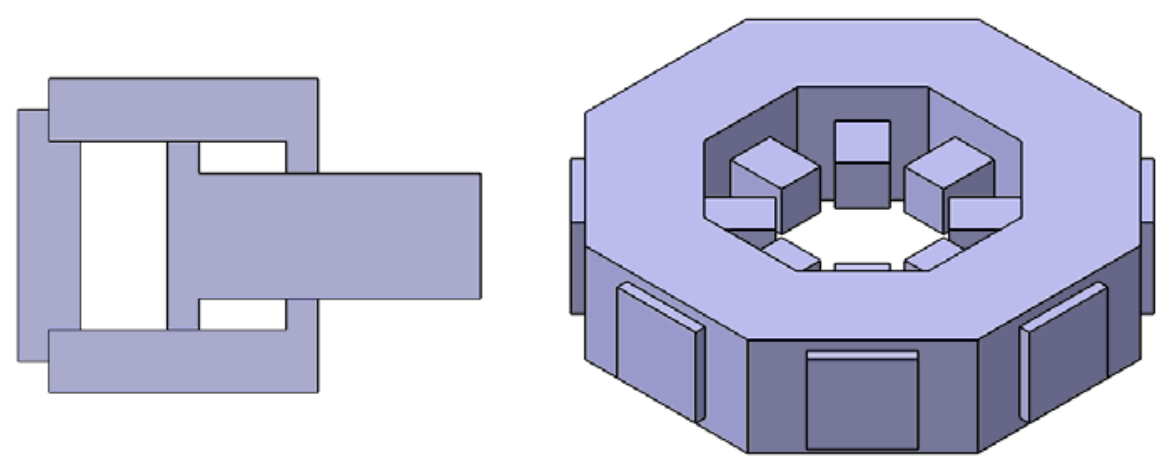

Figure 3.7 - Eight Piston Actuator Concept

The third radial actuator concept uses several out of the box components to actuate stiffness. Similar to the HFCDT's axial Festo cylinders these smaller cylinders are arranged such that they create pressure on the tool shaft pushing it to the center of the deburring tool, see Figure 3.8. Cylinders would be held onto the housing by a set screw, the actuating end would be attached to a contacting surface. The contacting surface would push against the tool shaft and provide stiffness to the shaft. The manufacturing cost of this design would be low due to the heavy reliance on off the shelf components. This design was quite large and would add to the size of the deburring tool substantially.

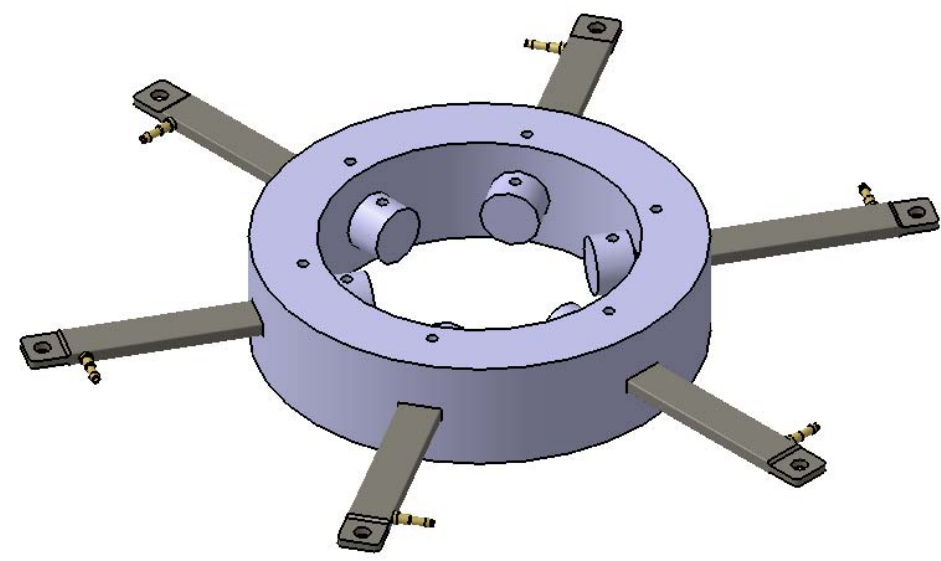

Figure 3.8 - Six Cylinder Festo Concept 
The fourth concept design is actuated via inflation of a series of rubber diaphragms. The rubber diaphragms were designed to be facing inward towards the deburring tool shaft. This idea was similar to the PRA in the HFCDT, with the exception that there are series of diaphragms instead of one large diaphragm surrounding the tool shaft. A cross-section in Figure 3.9 shows that the diaphragm would wrap-around a chamber that would be inserted into a housing. Figure 3.10 shows four, five, and six diaphragm versions of the design. This design could be adapted to any number of diaphragms. It is important to remember that the number of diaphragms adds to the complexity and cost of the actuator. The chamber could be fixed to the housing using a flange that steps past the housing and allows for a fastener to fix them together.

Manufacturing the wrap-around diaphragm design would be a series of complex $\mathrm{CNC}$ milling and possibly tooling, in order to achieve the dimensions, and angles required to accommodate the design. Assembly of the actuator would be difficult because stretching the diaphragm material over the chamber creates tension in the rubber material. The rubber would also resist insertion into the housing, as the friction of rubber and aluminum is significantly higher than that of two metals.

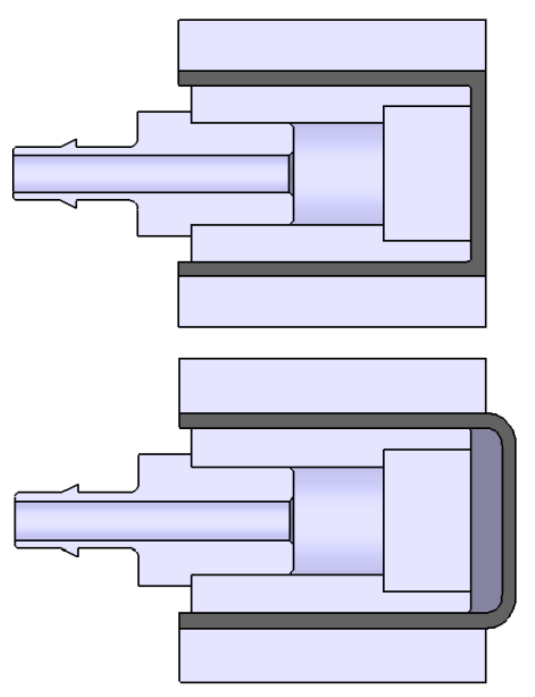

Figure 3.9 - Cross-section of wrap-around diaphragm concept 

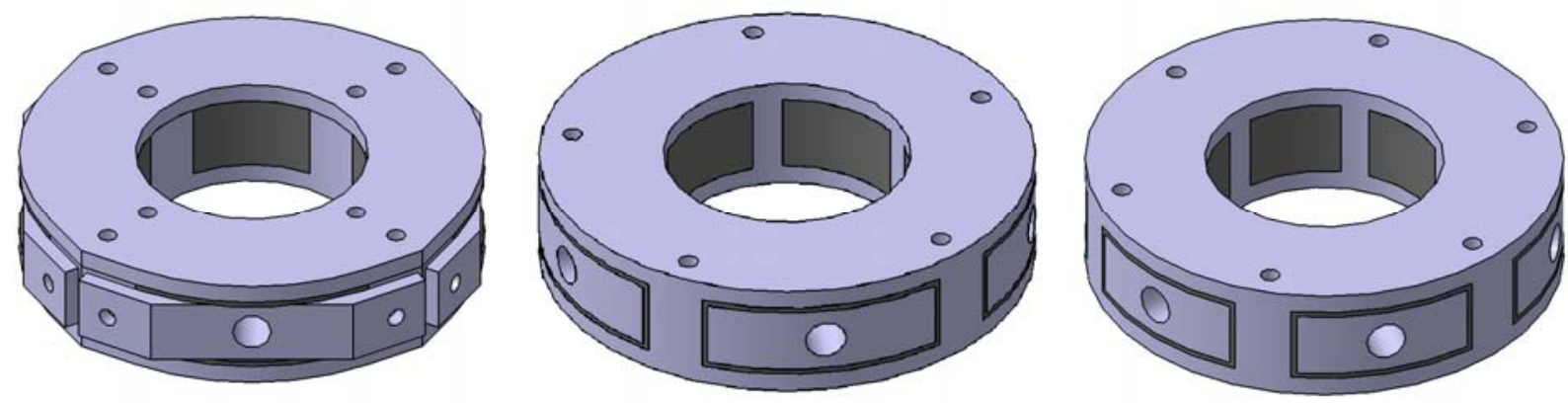

Figure 3.10 - Four, five, and six chamber wrap-around diaphragm concepts

The fifth concept explores the possibility of creating a single continuous diaphragm. The rubber diaphragm would have to consist of a single continuous elastic band. This would have to be specially manufactured and sourced, as it must be as homogenous as possible, to facilitate isotropic inflation. This concept utilizes two retaining rings that could be compression fit into two grooves on the inner diameter of the actuator housing, see Figure 3.11 for a diagram of the actuator.

Manufacturing the housing of the actuator would be simple as it could be turned down from a lathe and the holes could be drilled in a press. The rings would need to be spring steel heat treated for increased stiffness. The exact size of the rings would be difficult to determine due to the rings being compressed and placed into their grooves. This operation could require the rings to be cut to assist in their compressibility. The friction of the rubber diaphragm and the rings would have to be significant enough under the elastic spring force of the rings to resist the pressure of the expanding diaphragm. 

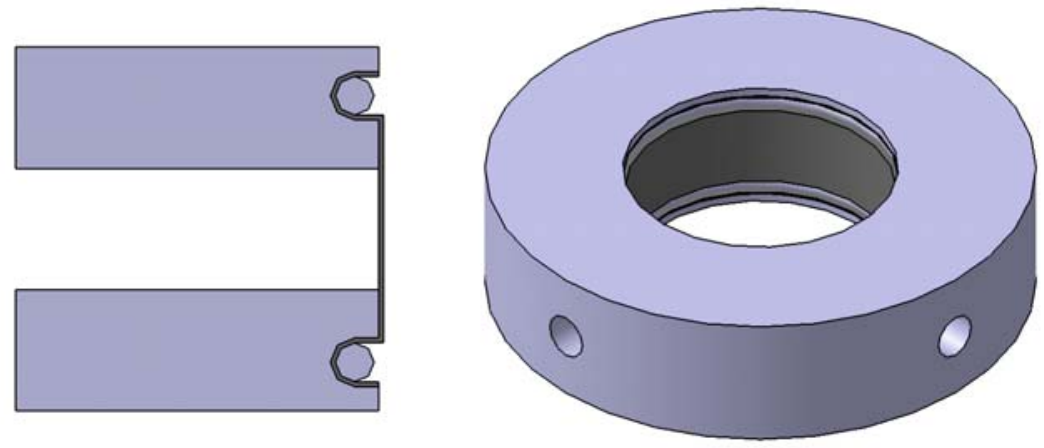

Figure 3.11 - Single Chamber Diaphragm with Inlaying Retaining Rings Concept

The sixth design concept is a variation of the fifth concept design. It utilized a single chamber diaphragm with two retaining rings that create an interference fit, see Figure 3.12 for a cross section and isometric view. Inflation of the diaphragm will result in the increased stiffness of the deburring tool in the radial direction. The change from a groved fit to an interference fit would simplify the assembly of the actuator, due to the retaining rings not requiring as much compression to be assembled. This concept would still be difficult to assemble, due to the requirement for the interference fitting retaining rings to resist the rubber diaphragm's natural tendency to slip from the housing and not maintain pressure.
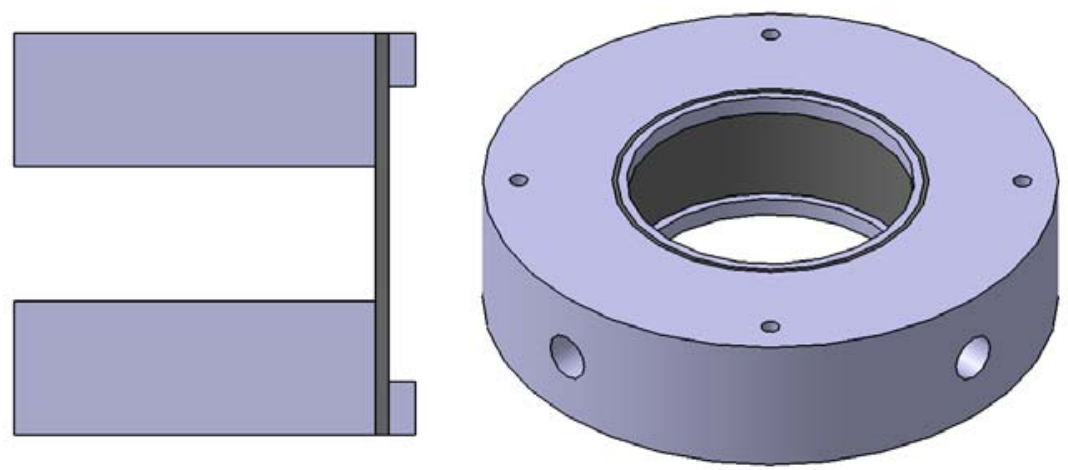

Figure 3.12 - Single Chamber Diaphragm with Interference Retaining Rings Concept

The seventh concept design takes another perspective to the multi-diaphragm concept. Using four diaphragms this concept takes a modular approach to the solution, see Figure 3.13 below. It was made up of four individual actuator housings, four diaphragms, four window retainers, and 
four fasteners each diaphragm. This design relys on the fastener or bolt to maintain pressure on the window shaped retainer to keep the diaphragm in place. The modular design allows for easy assembly and manufacturing of each component. The cost to manufacture this design would be relatively low as the components are simple to manufacture on a flat mill. Although the addition of the bolt to fasten the retainer was its strength, it was also its weakness. The hole required in the rubber diaphragm for the bolt would weaken it and was a stress riser in the highest stress portion of the diaphragm. The edge on the inside of the window retainer on the diaphragm saw the greatest stress. This design is likely to yield depending on pressures required.
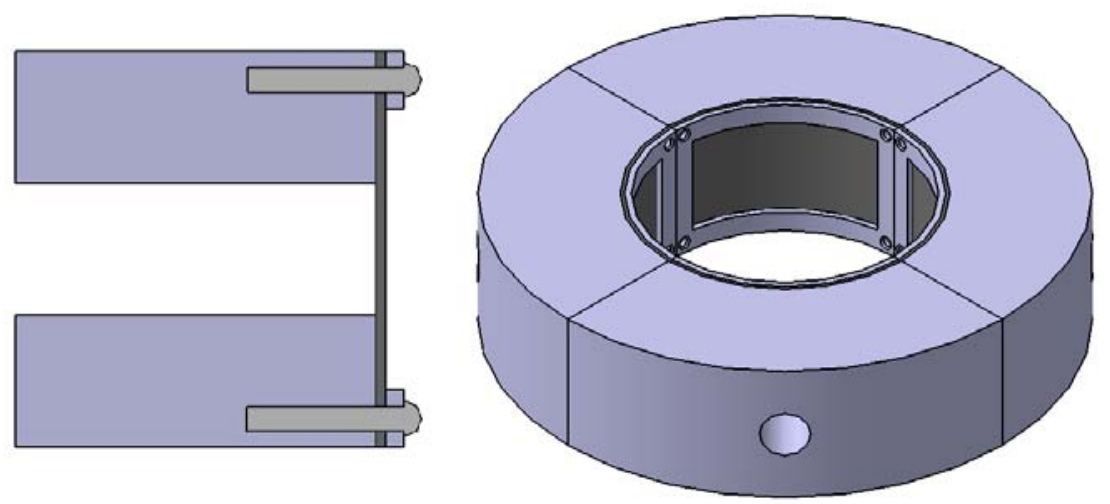

Figure 3.13 - Four Chamber Diaphragm with Window Retainers Concept

The eighth design concept draws on several of the previous concepts. It is a four chamber diaphragm actuator using retainer clips to clamp the diaphragm, see Figure 3.14 for a crosssection and isometric view of the actuator concept. This concept uses four ' $\mathrm{C}$ ' shaped retainer clips to apply a clamping pressure to the diaphragm in a window shape. The clamping force is maintained via countersunk machine screws on the top and bottom of the housing. The position of the retainers is adjustable via screw slots instead of finite screw holes. The diaphragm could be a continuous band, single strip around the inside of the housing, or four individual pieces of rubber. This gave the design added flexibility. The retainers sit into a groove in the housing 
allowing for a flush top and bottom of the actuator. This design could have been seamlessly installed on the current HFCDT. Manufacturing of this design will consist mostly of flat milling. The complex shape of the retainers would be difficult to develop due to their thin frame. The clamping force of the clips will be difficult to design for as the exact stack up of forces, torques, and friction would be complex.
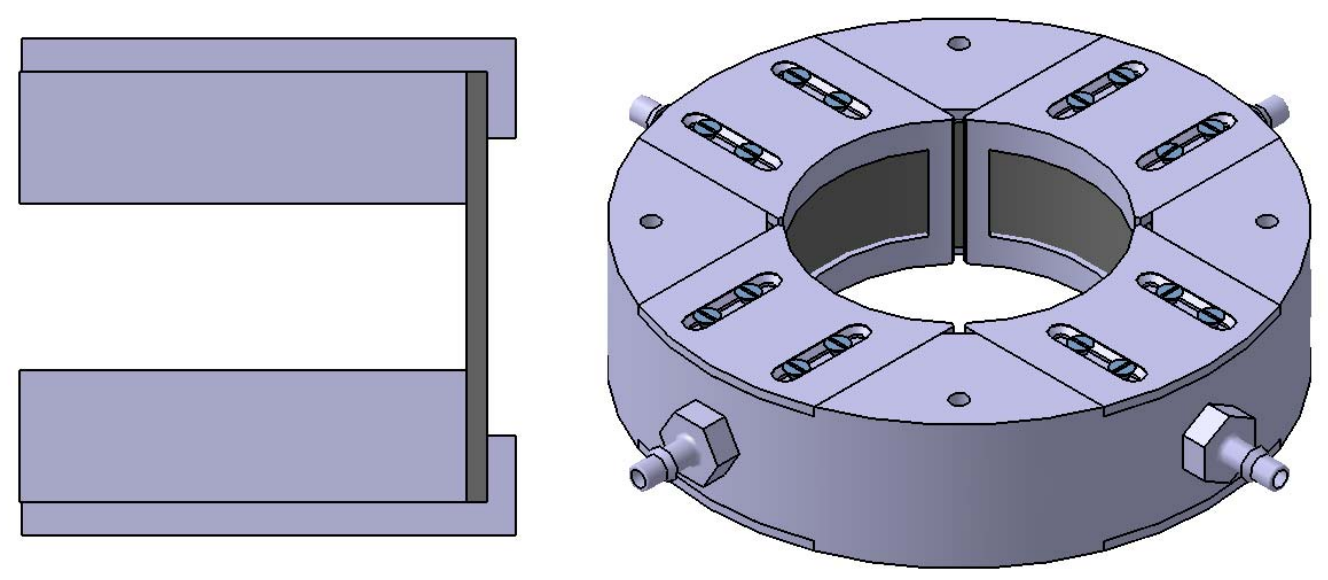

Figure 3.14 - Four Chamber Diaphragm with Retainer Clips Concept

\subsection{Trade Study}

A trade study was used to determine the best concept to continue into detailed design, manufacture, and testing. A comparison of the eight concepts was made by listing important characteristics that have been discussed. Each concept was given a score between 1 and 10 for each characteristic. Manufacturability describes the ease or difficulty of manufacturing the actuator, this includes operations required to machine and assemble. A higher ease of manufacturing would receive a higher score whereas an increased difficulty would receive a lower score. Patentability is a characteristic that is defined by previous work being completed on this concept's design features. If a concept contains features previously patented or researched for their application in this manner it will receive a lower score. New undocumented applications and designs will receive a higher score. Actuator and shaft contact is a reference to the possible 
effectiveness the actuator will have with contacting the shaft for translating stiffness of the actuator to the tool shaft. A higher score is more effective at achieving this. Tolerance is a reference to high tolerances or significance to the tolerance of the actuator's components to work effectively. Concepts requiring less attention to tolerance receive a higher score. Assembly is reference to the operations needed to complete the actuator that can be or may be required to be repeated. Some assembly of the actuator may be required to be repeated to adjust the performance of the actuator or for maintenance. Increased difficulty achieving a complete actuator receives a lower score. Complication is a reference to possible jamming or failure of the actuator to perform as expected. Concepts with lower possibility of failure will receive a higher score. Concepts with a higher cost associated with them will receive a lower score while lower cost concepts will receive a higher score, costs include manufacturing and maintenance.

All concepts were designed to provide compliance so the trade study has determined based on execution of the design which concept would be the most effective. According to Table 3.1 concept eight should be chosen for it superior manufacturability, its original design, its relatively simple design, and low cost. This concept will continue to detail design, manufacturing, and testing.

\begin{tabular}{|l|c|c|c|c|c|c|c|c|}
\hline & $\begin{array}{c}\text { Manufactur } \\
\text {-ability }\end{array}$ & Patentability & $\begin{array}{c}\text { Actuator } \\
\text { / Shaft } \\
\text { Contact }\end{array}$ & Tolerance & Assembly & Complication & Cost & Total \\
\hline 1 & 6 & 3 & 7 & 3 & 5 & 3 & 6 & 33 \\
\hline 2 & 7 & 5 & 7 & 4 & 6 & 6 & 7 & 42 \\
\hline 3 & 8 & 2 & 7 & 8 & 8 & 7 & 9 & 49 \\
\hline 4 & 5 & 9 & 8 & 3 & 2 & 4 & 4 & 35 \\
\hline 5 & 6 & 7 & 9 & 5 & 4 & 7 & 7 & 45 \\
\hline 6 & 6 & 7 & 9 & 5 & 5 & 6 & 8 & 46 \\
\hline 7 & 8 & 9 & 8 & 8 & 8 & 5 & 8 & 54 \\
\hline 8 & 8 & 9 & 8 & 7 & 7 & 8 & 9 & 56 \\
\hline
\end{tabular}

Table 3.1 - Trade study for radial actuator concepts 


\subsection{Developing the Enhanced Radial Actuator}

A thorough trade study was conducted on eight radial actuator concepts to arrive at a final concept to be designed in detail, manufactured, and tested. The concept chosen requires several details to be established. To fit directly onto the existing deburring tool housing the radial actuator was made roughly similar in size to the existing PRA. Four mounting holes were kept in the same position and dimension as the PRA. The enhanced radial actuator (ERA) was made to be 0.75 in high to keep the sensor and magnet distances approximately the same as they were previously designed to be. The height was also determined to keep the sensing side of the tool shaft directly centered with the diaphragms. This was to ensure that the inflated diaphragm contacts the tool shaft as symmetrically as possible. See Figure 3.15 for a cross-section of the inflated diaphragm contacting the tool shaft.

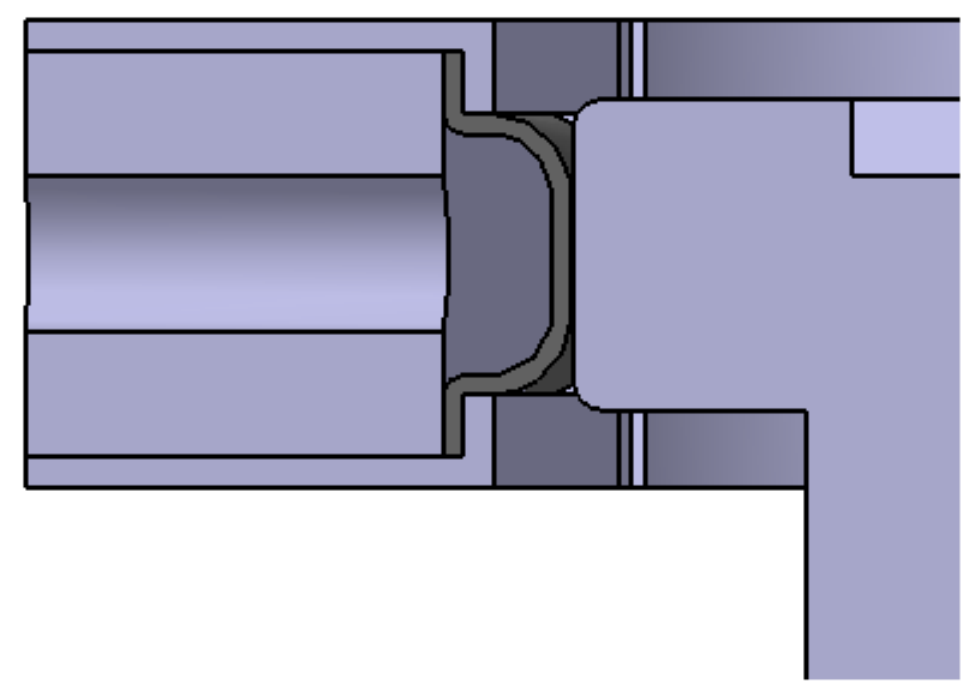

Figure 3.15 - Cross-section of inflated diaphragm contacting tool shaft

The inner diameter of the actuator was chosen to be smaller than the previous PRA. This was done to minimize the required amount of inflation of each diaphragm, and to maximize the stiffness that each diaphragm could produce. Each retainer was responsible for restricting the 
displacement of the diaphragm past the window of each diaphragm. This was done to allow the diaphragm to maintain pressure, create displacement and contact with the tool shaft, and to achieve a desired stiffness. It was important that the retainer was able to resist the maximum pressure required of the diaphragm. At Ryerson University the maximum air pressure available using the wall air supply system is 100 psi or approximately $690 \mathrm{MPa}$. It was taken into account that this is the standard in most manufacturing facilities so it would be an ideal maximum value to design for. In the concept presented the top and bottom flange of the retainer can have machine screws in them. At a minimum the retainer required to have one machine screw in the top and one in the bottom. It was important to remember that the screws were to be countersunk. This was to ensure that the ERA will fit onto the original tool design.

The thickness of the top and bottom flange was thick enough to contain the countersunk machine screws and additional material for the shank of the screw to rest against, without stress concentration, or knife edge condition. See Figure 3.16 for a diagram of a countersunk machine screw dimensions. The difference in the depth of the countersink and the total thickness was to be at least 0.005 in as a best practice. M1.6 countersunk machine screws have a countersink depth of $0.028 \mathrm{in}$.

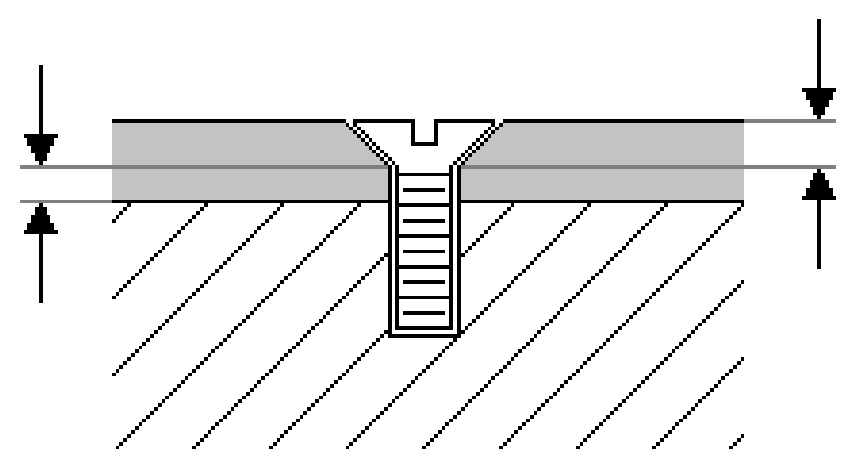

Figure 3.16 - Countersunk machine screw 
The face of the retainer which contacts the rubber diaphragm required dimensioning as it was interdependent on the thickness of the top and bottom retainer flange. Decreasing the thickness of the retainer flanges resulted in a larger diaphragm face contact area; this in turn reduced the chances of the diaphragm slipping from a clamping condition. See Figure 3.17 for a view of the contact surface of the diaphragm retainer.

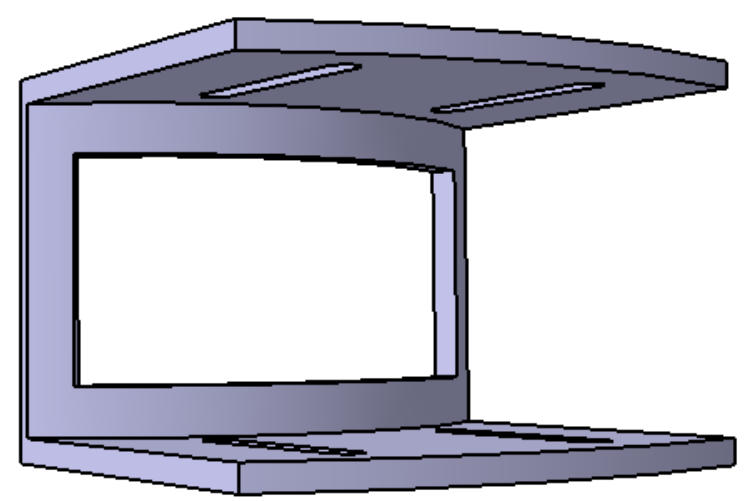

Figure 3.17 - Contact surface of diaphragm retainer

The maximum pressure of $690 \mathrm{MPa}$ was distributed to the contact surface of the diaphragm retainer. The force associated with this pressure was determined through the surface area. Based on four diaphragms equally spaced, each having 70 degrees of coverage the surface area of $2.025 \times 10^{-4} \mathrm{~m}^{2}$ was conservatively determined based on the available volume constraints. The force acting on the surface of contact between the retainer flange and the diaphragm block is approximately $140 \mathrm{~N}$. Assuming that the top and bottom flange equally distribute the force, each must resist a 70N shear force through friction applied via normal force of the machine screws. Applying a safety factor of two each flange should be able to withstand a $140 \mathrm{~N}$ shear force. It was clear that micro-fasteners would be required to fulfill dimensional requirements of the retainer, as the thickness of the retainer flange was approximated to be $0.050 \mathrm{in}$. M1.6 countersunk head machine screws were determined to be feasible dimensionally for the retainer based on having 0.017 in of thickness past the countersink. 
To determine if the machine screw was feasible for maintaining the clamping pressure on the diaphragm the normal force had to of be evaluated for a single screw. The normal force is equal to the torque of the screw over the nominal diameter multiplied by a friction coefficient. The torque required for an M1.6 machine screw is $0.059879 \mathrm{~N} \cdot \mathrm{m}$, the nominal diameter is $1.6 \mathrm{~mm}$ and the friction coefficient of steel on aluminum is 0.61 . This would yield a normal force of $61 \mathrm{~N}$ which was less than the required force. Three machine screws would suffice in each flange however for symmetry four machine screws will be installed on each flange of the diaphragm retainer.

The displacement of the tool shaft at the sensor end determined the available displacement at the tool tip. The smaller volume of the actuator must meet the requirement of the tool tip displacement. The possible displacement in the ERA is $\pm 3.175 \mathrm{~mm}$. The difference in the lower and upper displacement of the tool between the axis of rotation of the gimbal is $\pm 0.772 \mathrm{~mm}$. This exceeded the minimum required displacement of $\pm 0.4 \mathrm{~mm}$.

Material selection for the rubber diaphragm was completed, considering a broad spectrum of candidates. Natural rubber was previously used on the PRA; it was a natural choice to compare other rubbers. Using a mock-up of the diaphragm, rubbers were sampled and clamped into position and inflated under a range of pressures to determine how appropriate they were for this diaphragm application. Refer to Figure 3.18 for images of the diaphragm mockup. After a number of inflation tests were completed it was determined that silicone rubber at a thickness of 0.032in was deemed best suited for the actuators diaphragm based on inflation under a pressure range of 0-100psi. According to Bever [19] in their review of silicone rubber, the material has high flexibility at low temperatures as well as a high surface tension making it ideal for inflating and maintaining stiffness during contact with the tool shaft. 


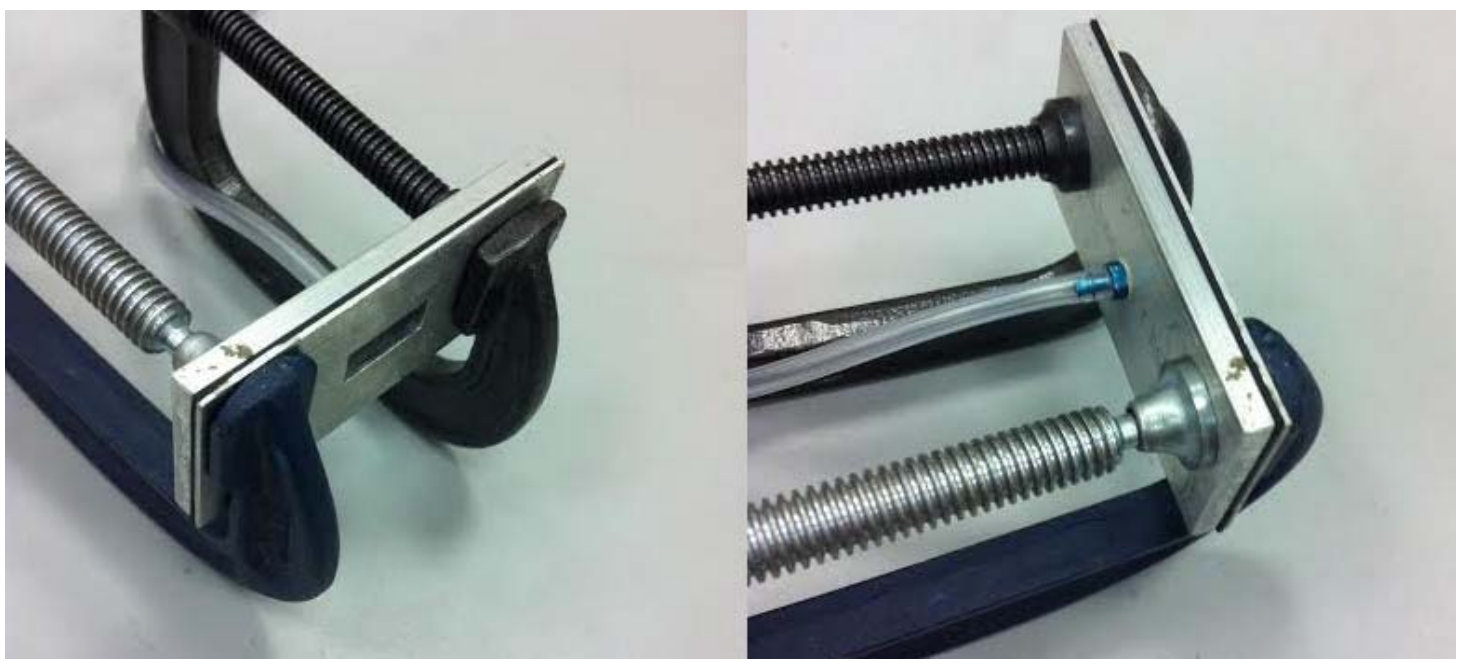

Figure 3.18 - Mockup of rubber diaphragm

\subsection{Sensor Design}

It was established that the design of the actuator allowed for the tool shaft to meet the requirement for minimum displacement. The previous magnetic sensor design was found to be quite effective for its application as they continuously produced a displacement signal. However the sensors required further testing and calibration for implementation in the deburring tool. The Honeywell HMC1501 magnetic field sensors [20] are composed of four anisotropic magnetoresistant (AMR) sensing elements configured into a Wheatstone bridge circuit. As magnetic field lines pass through the AMR elements, the current flowing through them causes a variance in impedance across the elements which will result in an associated voltage. As the angle of incidence varied with respect to the current, the voltage changed corresponding to a continuous output of measurable displacement.

The resolution of the HMC1501 was defined by the analog to digital data acquisition device used. A USB-1208FS by Measurement Computing was used to translate the analog signal to digital. The USB-1208FS unit has 12-bits of resolution. The magnetic sensors have a $\pm 120 \mathrm{mV}$ output amplified by the circuit by a factor of 39. The magnification of the circuit gives the sensor an output range of 9.36V. 12-bits of resolution would result in a resolution of $2.28516 \mathrm{mV}$. To 
establish the displacement resolution of the sensor a relationship between the voltage and displacement was determined. 


\section{Chapter 4}

\section{Analysis}

Modelling the deburring tool is a complex series of interacting systems. The tool's dynamic behaviour can be explained in a global and tool frame sense with additional detail for the tool tip and workpiece interaction and the tool shaft and actuator interaction. The tool tip and workpiece interaction will be explained through contact mechanics and abrasive cutting theory. The tool shaft and the actuator will be explained as a hyperelastic diaphragm contacting a solid aluminum shaft. A model will be presented to the behaviour of this interaction.

\subsection{Tool Modelling}

To determine the dynamic model of the tool the global coordinate system had to be first established. Further work to integrate this tool onto a robot for tool path planning will use this convention to determine the actual position of the deburring tool. The gimbal was selected to be the location of the origin of the tool due to this position remaining constant over the tool's range of motion. The two gimbal axes were selected to be the global $\mathrm{X}$ and global $\mathrm{Y}$ axes. The inner gimbal axis of rotation is the $\mathrm{X}$ axis and the outer gimbal axis is the $\mathrm{Y}$ axis. The global $\mathrm{Z}$ axis is in line with the axial direction of the deburring tool with the positive direction extending from the gimbal to the tool tip. See Figure 4.1 for a diagram of the global coordinate system for the deburring tool. The red axis indicators signify the positive side of each axis. 


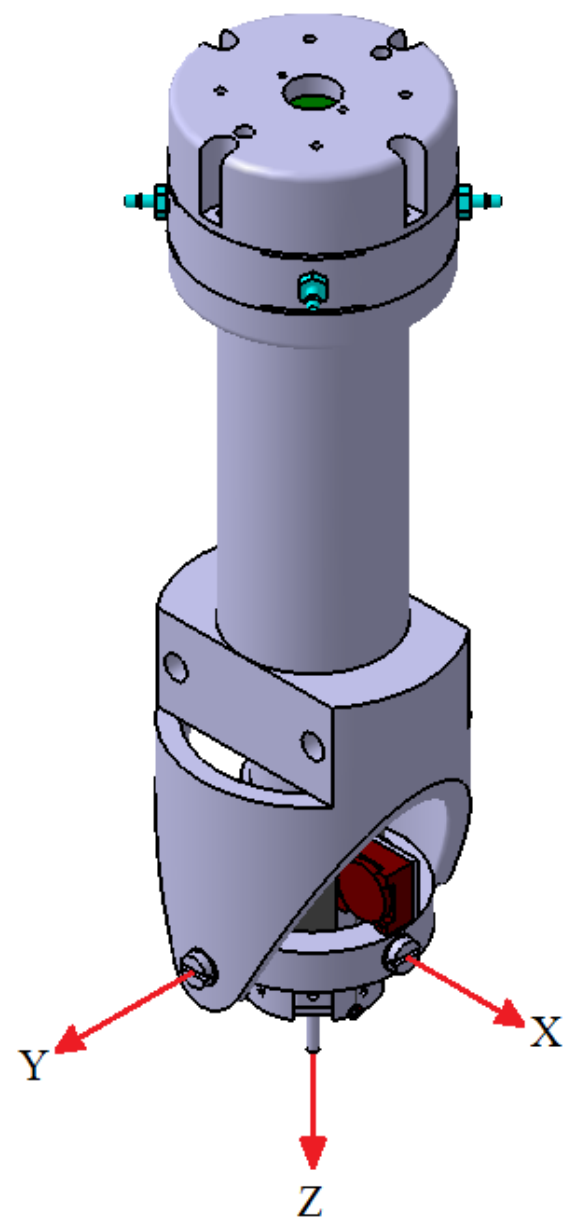

Figure 4.1 - Global coordinate system of the deburring tool

The deburring tool is further defined by an action plane referring to Figure 3.3 in section 3.1 . The tool's $\mathrm{x}$ and $\mathrm{y}$ axes are combined into a radial action plane. The action plane rotates parallel to the displacement/force. During radial actuation of the tool the action plane will describe the tool's full range of motion during manipulation of the radial actuator. See Figure 4.2 for a model of the deburring tool within the action plane. 


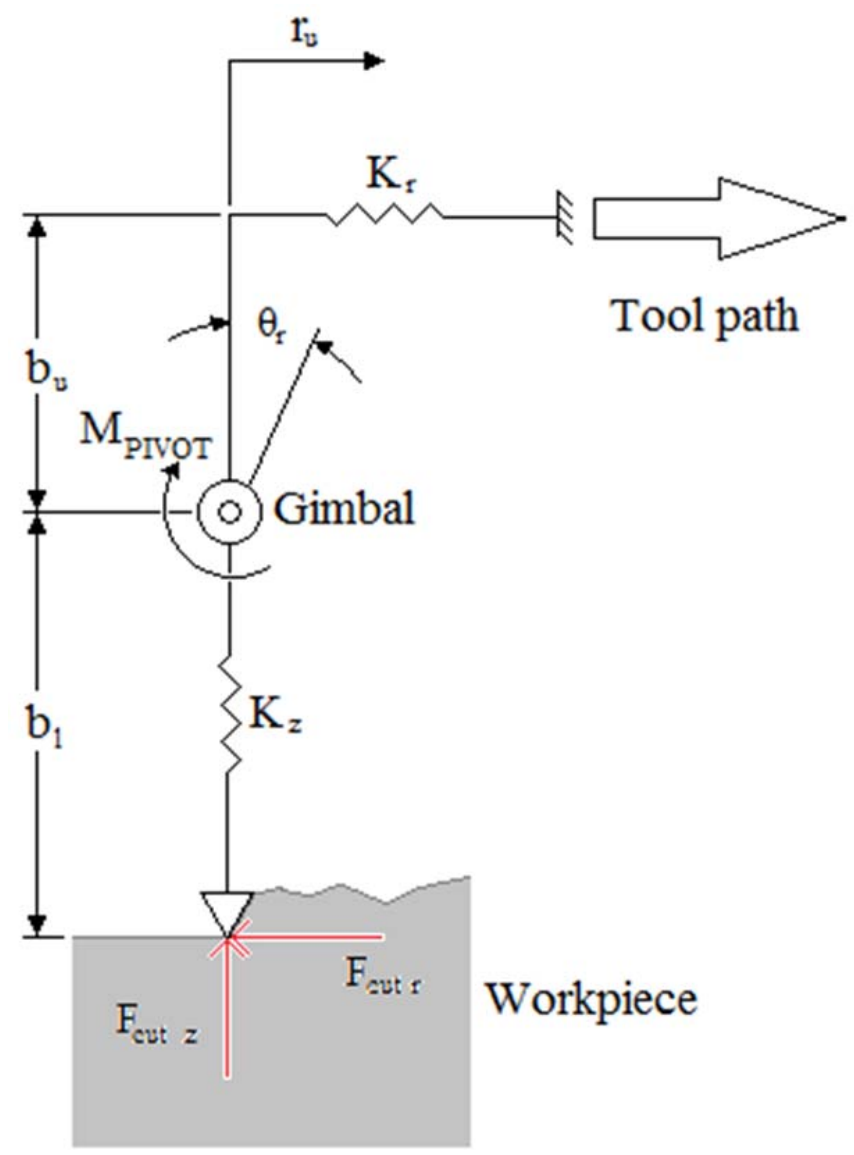

Figure 4.2 - Model of deburring tool in action plane

The tool has two displacements in the radial direction, $r_{l}$ is the lower radial displacement and $r_{u}$ is the upper radial displacement. The lower displacement is the displacement of the tool shaft as a direct result of an encountered burr. The upper displacement is the resultant displacement of the tool shaft at the sensor as the tool tip encounters a burr. The tool is split into an upper and lower half, $b_{l}$ is the distance from the gimbal to the tool tip and $b_{u}$ is the distance from the gimbal to the center of contact of for the tool shaft and actuator diaphragm. A relationship can be made between the four variables as can be seen in equation (4.1).

$$
r_{l}=\frac{b_{l}}{b_{u}} r_{u}
$$


The velocities and accelerations of the upper and lower radial displacements can be expressed by taking the derivative of (4.1) and can be seen in equations (4.2) and (4.3).

$$
\begin{aligned}
& \dot{r}_{l}=\frac{b_{l}}{b_{u}} \dot{r_{u}} \\
& \ddot{r}_{l}=\frac{b_{l}}{b_{u}} \ddot{r_{u}}
\end{aligned}
$$

The angle of rotation of the tool shaft in the action plane $\theta_{r}$ can be determined by taking the sine of the components for upper and lower sides of the tool. See equation (4.4) for the relationship.

$$
\sin \theta_{r}=\frac{r_{u}}{b_{u}}
$$

Due to the scale of the components a small angle approximation can be made making (4.4) become (4.5).

$$
\theta=\frac{r_{u}}{b_{u}}
$$

Angular velocity and acceleration can be expressed by taking the derivative of (4.5) to yeild (4.6) and (4.7) respectively.

$$
\begin{aligned}
& \dot{\theta}=\frac{\dot{r_{u}}}{b_{u}} \\
& \ddot{\theta}=\frac{\ddot{r_{u}}}{b_{u}}
\end{aligned}
$$

A summation of the moments about the axis of pivot $\left(M_{P I V O T}\right)$ is taken and can be seen in equation (4.8).

$$
M_{\text {PIVOT }}=M_{R A}+M_{\text {Cut }}
$$

$M_{R A}$ is the moment about the pivot caused by the radial actuator. $M_{C u t}$ is the moment about the pivot caused by the cutting force of the tool tip on the workpiece. Equation (4.9) further breaks $M_{R A}$ into its respective components the force of the radial actuator $\left(F_{R A}\right)$ multiplied by the 
moment arm $\left(b_{u}\right)$. The force of the radial actuator can be further broken down into the stiffness of the radial actuator $\left(K_{r}\right)$ multiplied by the radial displacement $\left(r_{u}\right)$ it has travelled.

$$
M_{R A}=F_{R A} b_{u}
$$

The moment about the pivot for the cutting force is similar to (4.9). They have different moment arms and therefore this yields equation (4.10).

$$
M_{\text {Cut }}=F_{r-C u t} b_{l}
$$

Substituting equations (4.9) and (4.10) into (4.8) and rewriting $M_{\text {PIVOT }}$ in terms of inertia will yield equation (4.11).

$$
\left(\frac{I}{b_{l}}\right) \ddot{r}_{l}+\left(K_{r} \frac{b_{u}^{2}}{b_{l}^{2}}\right) r_{l}=F_{r-C u t}
$$

Similarly, the axial modelling for the tool is the coupling of the actuator's stiffness with the tool tip and workpiece stiffness relationship. Equation (4.12) gives the dynamic relationship between the mass of the moving axial component of the tool and the stiffness relationship in the axial direction.

$$
m_{z} \ddot{z}+K_{z} z=F_{z-C u t}
$$

The combination of these two models yields equation (4.13).

$$
\left[\begin{array}{cc}
\frac{I}{b_{l}} & 0 \\
0 & m_{z}
\end{array}\right]\left(\begin{array}{l}
\ddot{r}_{l} \\
\ddot{z}
\end{array}\right)+\left[\begin{array}{cc}
K_{r} \frac{b_{u}{ }^{2}}{b_{l}{ }^{2}} & 0 \\
0 & K_{z}
\end{array}\right]\left(\begin{array}{l}
r_{l} \\
z
\end{array}\right)=\left\{\begin{array}{l}
F_{r-C u t} \\
F_{z-C u t}
\end{array}\right\}
$$

Each term is an important part of the dynamic model of the deburring tool. In many cases another model is used to define a term in (4.13). The terms $F_{r-C u t}$ and $F_{z-C u t}$ are defined by the tool / workpiece interaction. This interaction is defined by hertzian contact mechanics. Section 4.2 will define contact mechanics theory and how it will define these terms.

The term $K_{z}$ has been well defined by Liao [11] on the active force compliant axial deburring tool. $K_{Z}$ is a combination of the spring force and the force of the air pressure in the pneumatic 
cylinders. The air pressure force is a simple relationship. It is modelled by the force being equal to the pressure in the cylinder multiplied by the surface area of the piston. The pneumatic cylinder is a simple linear actuator and the displacement of the moving component can be determined from the string pot. The spring stiffness of the actuators can be measured under ambient pressure conditions.

The term $K_{r}$ is the stiffness of relationship of the radial actuator. The stiffness of the radial actuator is a function of the forces acting upon it and the resultant displacement of the resultant force. The diaphragm is made from a non-linear elastic material. These materials are usally modelled as hyperelastic materials. There are many models that have been determined throughout research on the subject. Both the pressure on the diaphragm and the tool shaft will contribute to the displacement of the actuator. This will be modelled in detail in section 4.3 and simulated in section 4.4 .

\subsection{Tool and Workpiece Interaction}

Contact between the workpiece and tool define the force required for the tooltip to remove a required amount of material from the workpiece. In this case the required amount of material is the burr or edge of the part which requires deburring or chamfering. Hertzian contact is a reference Heinrich Hertz's original research on the optical properties of multiple, stacked lenses, with a force holding them together. Currently this theory is applied to a number of geometric variations. For the contact of a burr with the asperity of an abrasive tool tip the model of two discs contacting with radii $R_{1}, R_{2}, R_{1}^{\prime}, R_{2}^{\prime}$, and applied force $F$ which is the force encountered by the tool as it encounters the workpiece. See Figure 4.3 for geometry of the Hertzian disc contact model. 


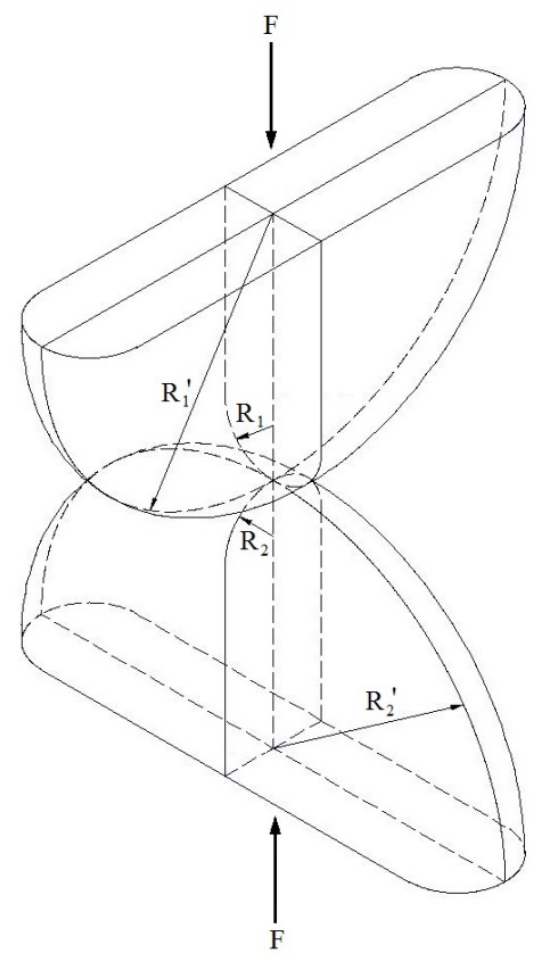

Figure 4.3 - Hertzian disc contact geometry

Several fundamental assumptions are made about the materials involved and the geometry of contact. The properties of each body are homogenous, isotropic, and elastic in accordance with Hooke's law. The two bodies are not necessarily made of the same material. The shape of the surfaces near the point of contact before loading of the two bodies is in contact at a point, and there is a common tangent plane to the surfaces at the point of contact. The total minimum distance $d$ between corresponding points on any two surfaces is equal to (4.14). See Figure 4.4 for a diagram of the tangent plane between two Hertzian discs.

In Hertz's original research, a relationship between the two corresponding points in Figure 4.4 was defined as the equation of an ellipse (4.15). See Figure 4.5 for the orientation of the elliptical contact between two discs.

$$
d=z_{1}+z_{2}
$$




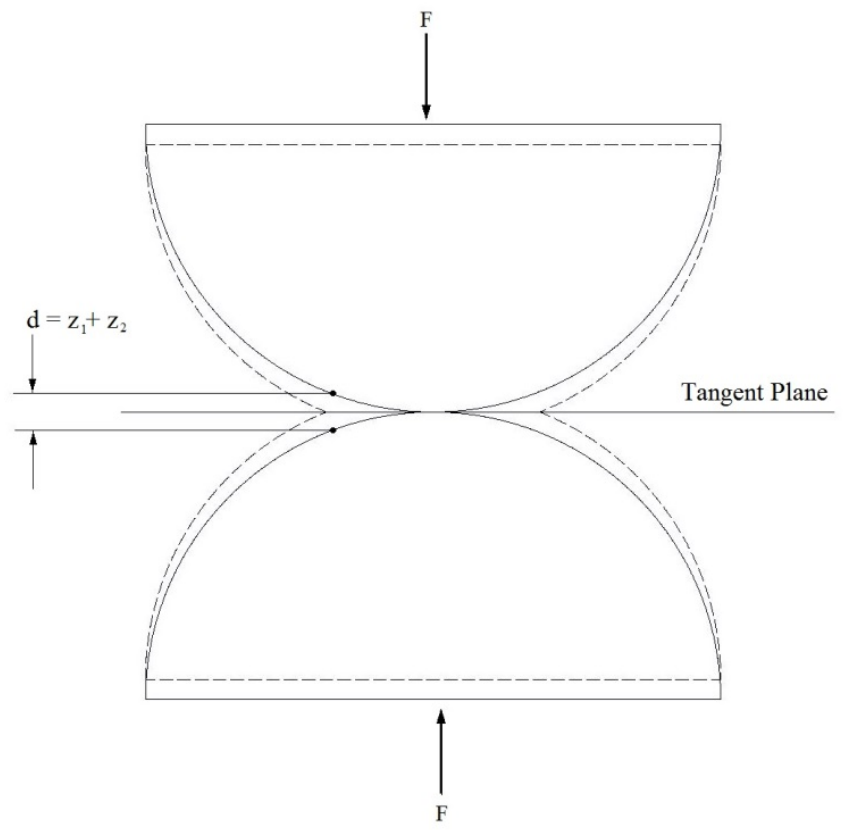

Figure 4.4 - Hertzian disc contact tangent plane

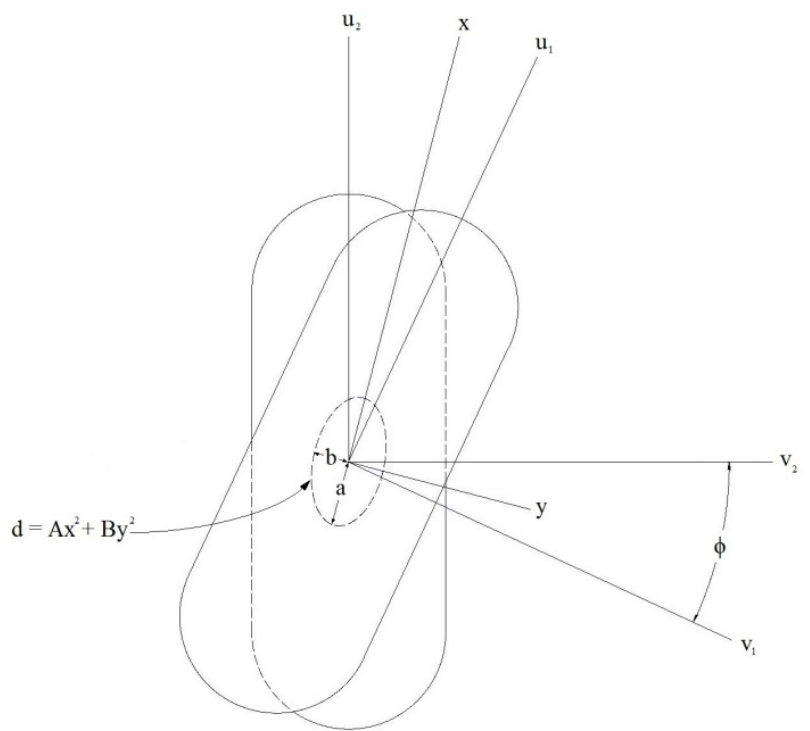

Figure 4.5 - Hertzian disc contact orientation

$$
d=A x^{2}+B y^{2}
$$


Through relating the geometry of the discs, the values of $z_{1}$ and $z_{2}$, and their relation to the tangent plane; a transformation is made to the form of (4.15) and it is found that $A$ and $B$ are the roots of a quadratic equation and yield (4.16) and (4.17).

$$
\begin{aligned}
& A=\frac{1}{4}\left(\frac{1}{R_{1}}+\frac{1}{R_{2}}+\frac{1}{R_{1}^{\prime}}+\frac{1}{R_{2}^{\prime}}\right)-\frac{1}{4} \sqrt{\left[\left(\frac{1}{R_{1}}-\frac{1}{R_{1}^{\prime}}\right)+\left(\frac{1}{R_{2}}-\frac{1}{R_{2}^{\prime}}\right)\right]^{2}-4\left(\frac{1}{R_{1}}-\frac{1}{R_{1}^{\prime}}\right)\left(\frac{1}{R_{2}}-\frac{1}{R_{2}^{\prime}}\right) \sin ^{2}(\phi)} \\
& B=\frac{1}{4}\left(\frac{1}{R_{1}}+\frac{1}{R_{2}}+\frac{1}{R_{1}^{\prime}}+\frac{1}{R_{2}^{\prime}}\right)+\frac{1}{4} \sqrt{\left[\left(\frac{1}{R_{1}}-\frac{1}{R_{1}^{\prime}}\right)+\left(\frac{1}{R_{2}}-\frac{1}{R_{2}^{\prime}}\right)\right]^{2}-4\left(\frac{1}{R_{1}}-\frac{1}{R_{1}^{\prime}}\right)\left(\frac{1}{R_{2}}-\frac{1}{R_{2}^{\prime}}\right) \sin ^{2}(\phi)}
\end{aligned}
$$

Stress in the $\mathrm{z}$ direction is well defined by research done by Thomas and Hoersch [21], see equation (4.18). Figure 4.6 shows the direction and orientation of the contact stress.

$$
\sigma_{z z}=-\left[\frac{M}{2}\left(\frac{1}{n}-n\right)\right] \frac{b}{\Lambda}
$$

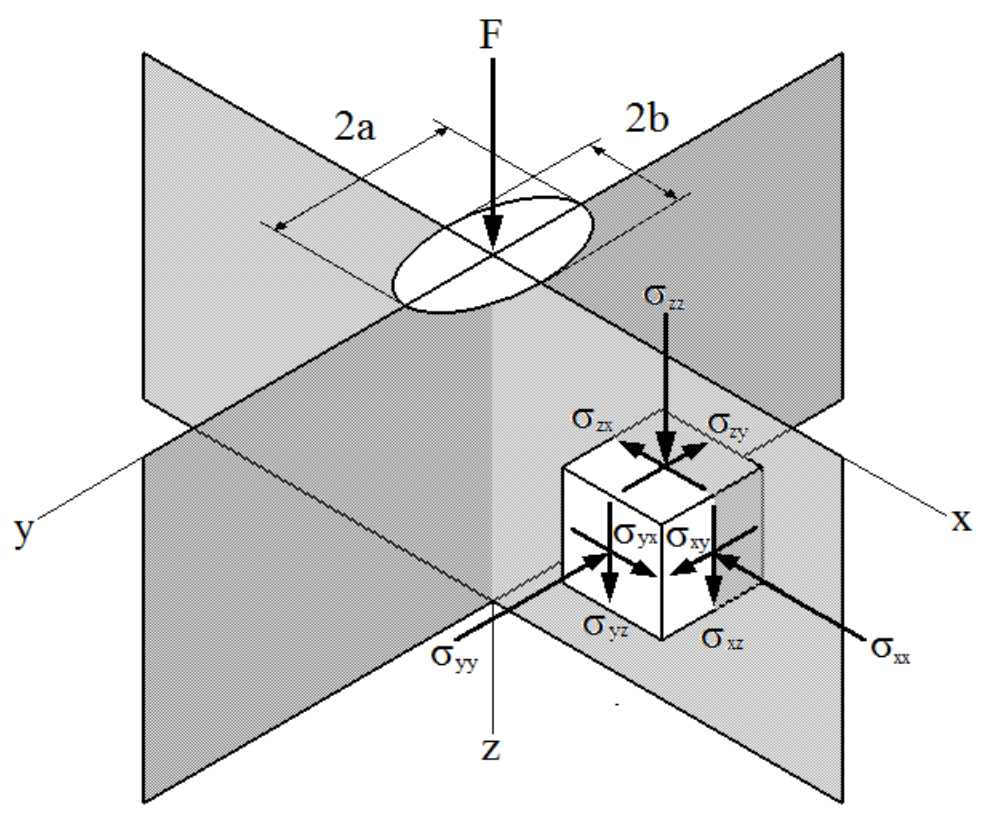

Figure 4.6 - Hertzian contact stress

The ratio of the semimajor axis to the semiminor axis of the ellipse of contact is denoted by $k$ see equation (4.19). The derivative of $k$ is $k^{\prime}$ in equation (4.20). $M, n$, and $\Lambda$ are values defined by Thomas and Hoersch are defined as equations (4.21), (4.22), and (4.23) respectively. $Z$ is the 
depth from the contact surface area along the z-axis of the disc. $\Lambda$ contains the material properties of each disc. The Poisson's ratio for each material is $v_{1}$ and $v_{2}$ for each disc respectively. The elastic modulus of each material is $E_{1}$ and $E_{2}$ for each disc respectively. $E\left(k^{\prime}\right)$ is a standard elliptical integral found in most mathematical handbooks, see equation (4.24).

$$
\begin{gathered}
k=\frac{a}{b}=\cos \theta \\
k^{\prime}=\sqrt{\left(1-k^{2}\right)}=\sin \theta \\
M=\frac{2 k}{k^{\prime 2} E\left(k^{\prime}\right)} \\
n=\sqrt{\frac{k^{2}+k^{2}(Z / b)^{2}}{1+k^{2}(Z / b)^{2}}} \frac{1}{A+B}\left(\frac{1-v_{1}^{2}}{E_{1}}+\frac{1-v_{2}^{2}}{E_{2}}\right) \\
E\left(k^{\prime}\right)=\int_{0}^{2 \pi} \sqrt{1-k^{\prime 2} \sin ^{2}(\theta)} d \theta
\end{gathered}
$$

The semiminor axis of the area of contact depends on equations (4.19) through (4.23) and on the load $F$ on the discs. Equation (4.25) expresses this relationship.

$$
b=\sqrt[3]{\frac{3 k E\left(k^{\prime}\right)}{2 \pi}(F \Lambda)}
$$

Considering the geometry of a burr we can assume $R_{1}$ and $R^{\prime}{ }_{1}$ to be infinity large when comparing the size of the cutting tool to the size of a burr. The contact pressure of the tool and burr can be defined by taking equation (4.18) and substituting (4.21) and (4.22) into it with $Z=0$. This yields equation (4.26). See Figure 4.7 for a diagram of the contact pressure distribution. 


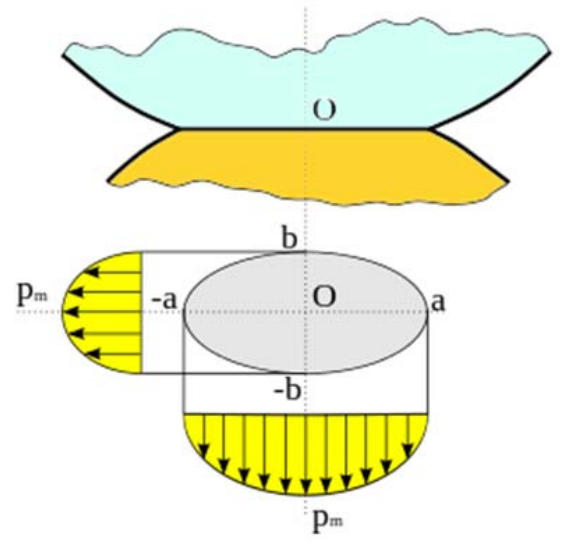

Figure 4.7 - Mean stress distribution across elliptical contact area [12]

$$
P_{0}=\left|-\frac{b}{E\left(k^{\prime}\right) \Lambda}\right|
$$

Substitute $\phi=0$ into (4.16) and (4.17). Dividing (4.17) by (4.16) to yields (4.27).

$$
\frac{B}{A}=\frac{R \prime_{2}}{R_{2}}
$$

The elliptical stress distribution pattern can be defined in (4.28). This equation is in the form of the equation of an ellipsoid. From this we can determine that the mean stress would be two thirds of the contact stress (4.29).

$$
\begin{gathered}
P_{\text {seg }}(x, y)=P_{0}\left[1-(x / a)^{2}-(y / b)^{2}\right] \\
P_{m}=\frac{2}{3} P_{0}
\end{gathered}
$$

Substituting (4.25) and (4.26) into (4.29) produces (4.30).

$$
P_{m}=\sqrt[3]{\frac{4 k F}{9 \pi E^{2}\left(k^{\prime}\right) \Lambda^{2}}}
$$

The applied force creating contact can be isolated from (4.30) to yield (4.31).

$$
F=\frac{9 \pi E^{2}\left(k^{\prime}\right) \Delta^{2} P_{m}^{2}}{4 k}
$$


$F$ is the force encountered by the tool as it encounters the part. In this case, it is the force the tool must exert for deburring.

\subsection{Tool and Actuator Interaction}

The actuator's primary function is to provide the deburring tool with radial compliance. To achieve this, the radial actuator has a 0.125 in gap between the unpressurized diaphragm and the centered outer diameter of the tool shaft which is fixed to a 2-axis gimbal. The gap between the diaphragm and the tool shaft will become filled with the inflated diaphragm upon a demand for pressure. When encountering a burr the tool tip will deflect and because of the 2-axis gimbal this will cause a radial deflection of the tool shaft within the action plane. The deflection will cause a reactant force on the radial actuator that will be realized by a diaphragm causing a deformation of this diaphragm. The total deformation of the tool shaft will be the resultant of the cutting force of the burr and the inflating of the diaphragm due to the air pressure.

A stiffness relationship is required of the actuator to determine its behaviour under pressure and contact from the tool shaft. The first consideration to determine the behaviour of the stiffness of the actuator is to determine which material model should be used for the diaphragm. Silicone rubber is not an isotropic non-linearly elastic material so it does not follow Hooke's for all values of strain. Materials like rubber deform non-linearly without yielding, these materials are called hyperelastic materials. Hyperelastic materials have a greater potential for storing energy than linearly elastic materials like metals and therefore require a model to define the energy relationship.

Hyperelastic materials are defined by a constitutive model which the stress-strain relationship derives from a strain energy density function. A strain energy density function is a scalar valued function that relates the strain energy density of a material to the deformation gradient tensor. The deformation gradient tensor is both related to the reference and current configuration of a 
body. Hyperelastic material constitutive models can be classified into three types; phenomenological, mechanistic, and hybrid. Phenomenological models are models that describe the observed behaviour of the material. Mechanistic models are derived from the idealized structure of the material. Hybrid models combine the two approaches. Each model has been found to better describe certain materials or strain ranges.

Impact engineering solutions make material model recommendations based on testing data of various hyperelastic materials. Based on their recommendation silicone rubber should be modelled with an Arruda-Boyce constitutive model. This can be supported by Arruda and Boyce [22] in their work, where they test and successfully model the material behaviour of silicone rubber for strains up to $300 \%$. According to Zheng [23] the Arruda-Boyce model is the most successful statistical mechanics model so far proposed. This is likely due to the eight-chain molecular network that it is modelled after. In an element of the model each node of the cubicrectangular element is fixed to a molecular chain of the silicone rubber and then all chains are fixed to the centre. See Figure 4.8 for a diagram of the eight-chain network model.

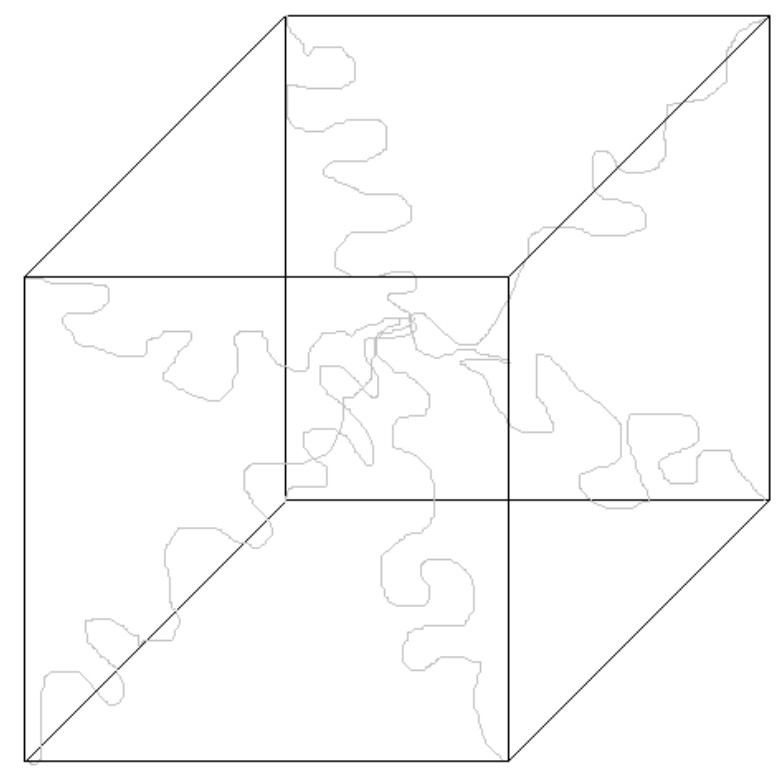

Figure 4.8 - Eight-chain network model 
The strain energy density function of the Arruda-Boyce model is derived as follows.

$$
\begin{gathered}
W_{8 c h}=\frac{N k_{B} T}{2}\left[\beta \lambda_{\text {chain }}+\sqrt{n} \ln \left(\frac{\beta}{\sinh \beta}\right)\right] \\
\lambda_{\text {chain }}=\sqrt{\frac{I_{1}}{3}} \\
\beta=L^{-1}\left(\frac{\lambda_{\text {chain }}}{\sqrt{n_{\text {chain }}}}\right)
\end{gathered}
$$

The number of chain segments is denoted by $n$, the first invariant of the left Cauchy-Green deformation tensor is denoted by $I_{1}$, Boltzmann constant is represented by $k_{B}$, temperature is represented by $T$, and the number of chains in the network of a cross-linked polymer is represented by $N$. The strain energy density formula can be represented in polynomial form in equation (4.35).

$$
W_{8 c h}=\mu \sum_{i=1}^{n}\left[\frac{C_{i}}{\lambda_{m}^{2 i-2}}\left(I_{1}^{i}-3^{i}\right)\right]
$$

The locking stretch of the material is represented by $\lambda_{m}$, and the initial shear modulus is represented by $\mu$. The strain energy density can be expressed in terms of the deformation gradient. See equation (4.36), note that Langevin function is expressed in the series expansion form for the first three terms.

$$
\bar{U}=\mu\left\{\frac{1}{2}\left(\bar{I}_{1}-3\right)+\frac{1}{20 \beta^{2}}\left(\bar{I}_{1}^{2}-9\right)+\frac{11}{1050 \beta^{4}}\left(\bar{I}_{1}^{3}-27\right)+\cdots\right\}+\frac{K}{2}(J-1)^{2}
$$

The stress-strain law for the Arruda-Boyce model is expressed in equation (4.37).

$$
\sigma_{i j}=\frac{\mu}{J^{5 / 3}}\left(1+\frac{I_{1}}{5 J^{2 / 3} \beta^{2}}+\frac{33 I_{1}{ }^{2}}{525 \beta^{4} J^{4 / 3}}+\cdots\right)\left(B_{i j}-\frac{I_{1}}{3} \delta_{i j}\right)+K(J-1) \delta_{i j}
$$

The principle stress-strain relationship seen in equation (4.37) can be used to define an engineering stress-strain relationship dependent on the type of deformation being modelled. This 
can be used in a finite element model to determine the solution to a defined system. However, the contact of the diaphragm and the tool shaft has not been defined. This is done through computation software by constantly updating the boundary conditions and causing local nonlinear deformation of the contacting elements. The contacting elements will then deform based on the reactant forces of contact. This is done through detection of penetration during load stepping towards a solution to the system.

\subsection{Simulation}

The pneumatically actuated diaphragm is a hyperelastic material pressurized by a dynamic pressure source. The deformed diaphragm contacts the tool shaft which is loaded due to the cutting force from the tool tip. The loading is scaled by the ratio of moment arm length between the top and bottom of the tool shaft. In the case of the tool the gimbal axis to the tool tip is represented by $b_{l}$ and measures to be $0.041021 \mathrm{~m}$. Measured from the same axis, the distance from the gimbal axis to the radial actuator is represented by $b_{u}$ and measures to be $0.225425 \mathrm{~m}$. Forces at the tool tip can be scaled down to the shaft force on the actuator. Due to the relatively small forces on the tool tip the tool shaft will not experience significant bending. The diaphragm / actuator geometry can be modelled as a single curved rectangular body contacting a cylindrical body. The curved rectangular body will be modelled with non-linear hyperelastic material elements whereas the cylindrical body can be modelled with linear material elements. See Figure 4.9 for a diagram of the tool and diaphragm model. 


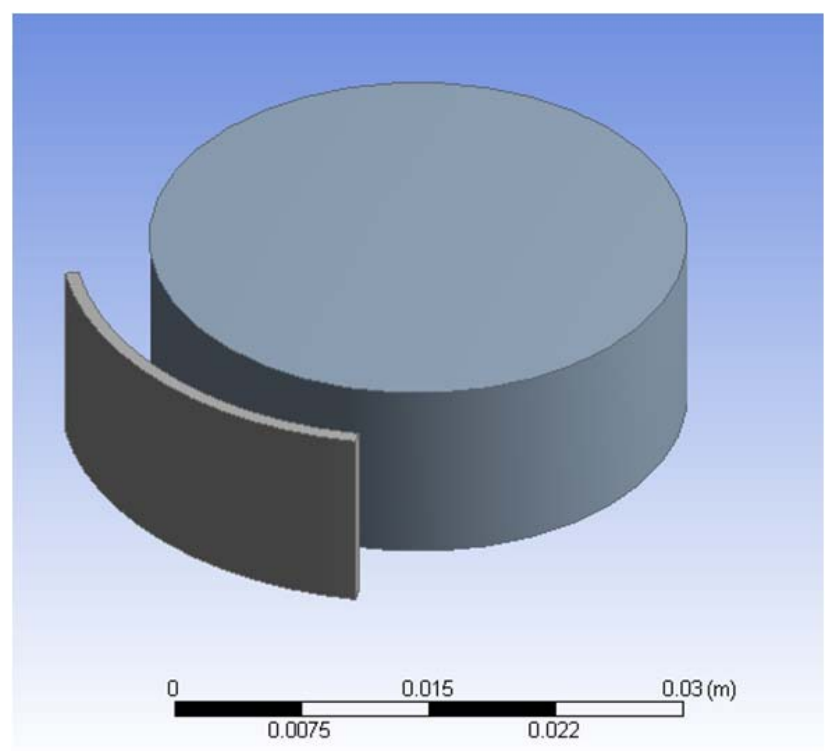

Figure 4.9 - Initial diaphragm / tool model

To simplify the analysis several assumptions can be made and applied. Symmetry can be applied to the system to reduce the effect that an unsymmetrical mesh will have on the solution. An unsymmetrical mesh could cause unnecessary resultant forces and torque on the model. See Figure 4.10 for a diagram of the tool shaft and diaphragm planes of symmetry. The surfaces in red indicate planes of symmetry normal to the surface.

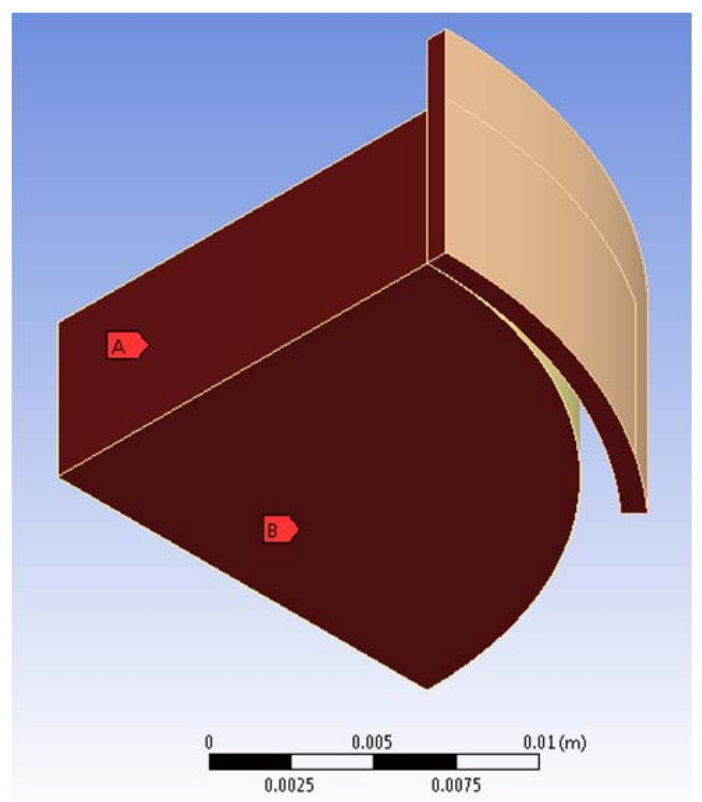


Figure 4.10 - Symmetry on the tool shaft / diaphragm model

The tool shaft will contact the diaphragm as there is nothing stopping it from doing so. Modelling the tool shaft initially contacting the diaphragm will be much easier to solve as impact analysis would have to be completed on the model to determine at what step the two bodies contact. See Figure 4.11 for a diagram of the tool shaft / diaphragm contact condition. The tool shaft surface in red will be the contact surface and the diaphragm surface in blue will be the target surface. A frictional contact condition of 0.5 is used as the frictional coefficient between silicone rubber and aluminum can vary between 0.25 and 0.75 .

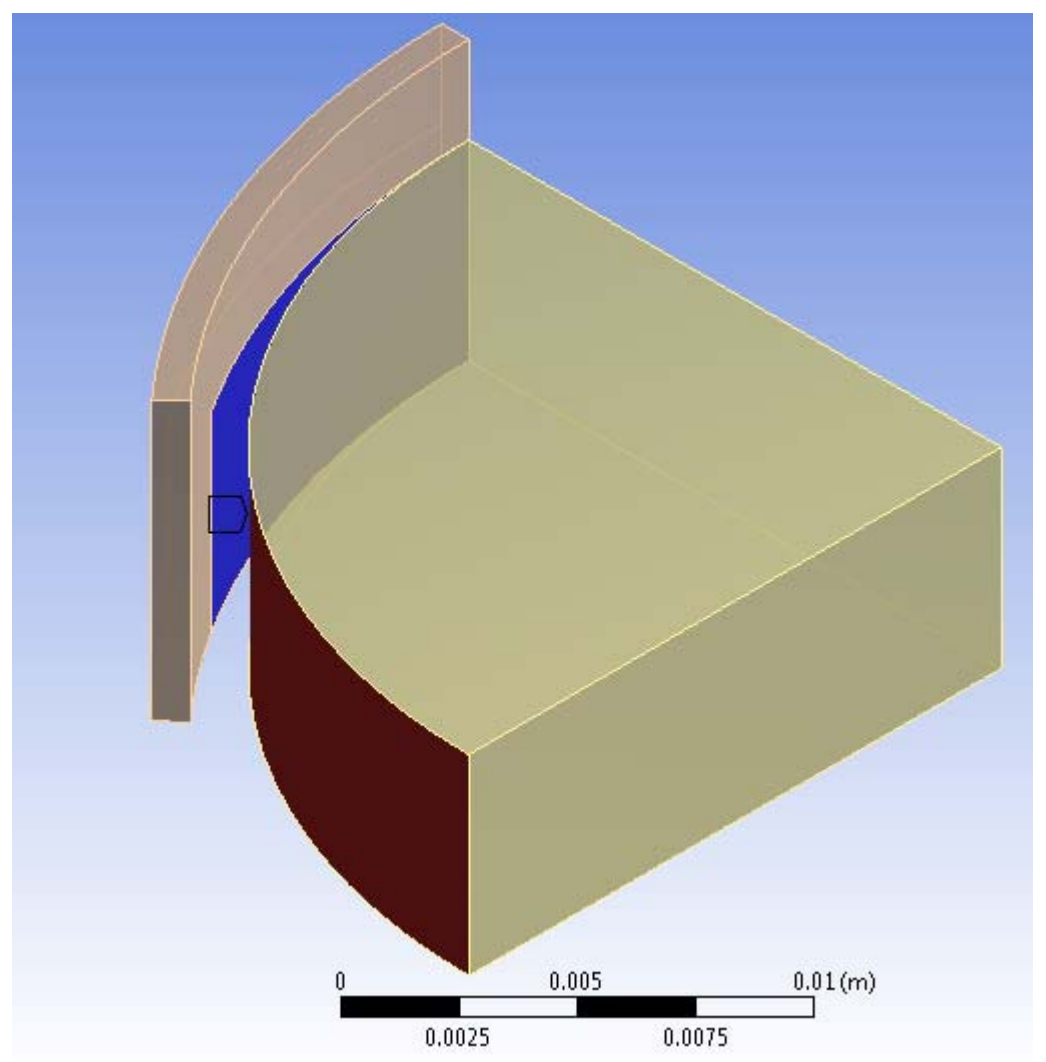

Figure 4.11 - Tool shaft / diaphragm contact condition

The clamping force on the diaphragm can be approximated by fixing the clamping surface to reduce under constraining of the model. See Figure 4.12 for a diagram of the diaphragm clamping conditions. Under constraining the model in this case would mean that the supports 
where reaction forces are calculated from and displacement no longer happens would need to be iterated to a solution for the clamping force and the frictional coefficient on that surface. This would be difficult to converge on a solution due to the added complexity it would add.

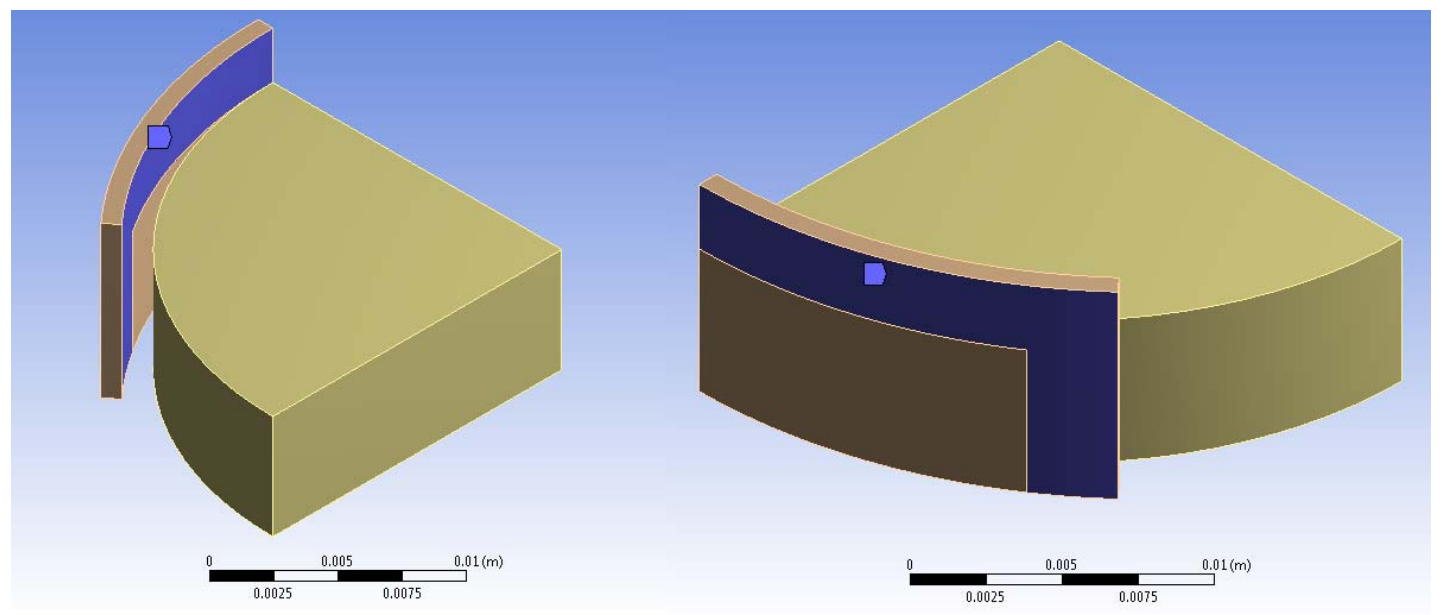

Figure 4.12 - Clamping conditions on diaphragm

The mesh should be created using three dimensional brick elements as opposed to tetrahedral element due to the thin dimensions of the diaphragm. This will provide more consistency with solution results. Using brick elements is also ideal for mesh contact region as tetrahedral elements will be difficult to compute resulting contact conditions. See Figure 4.13 for a diagram of the FEM model mesh. 


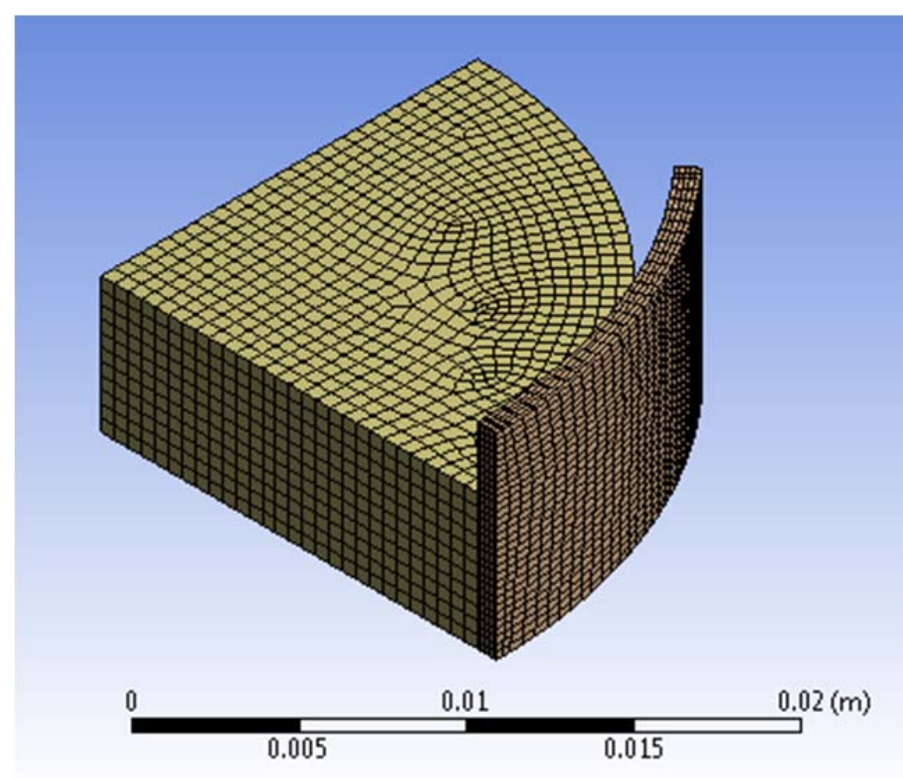

Figure 4.13 - Finite element model mesh

The force of the tool shaft on the diaphragm will be applied to the flat face of the tool shaft cylinder, while the pressure will be applied to the free surface opposite to the contact of the cylinder. See Figure 4.14 for a diagram of the pressure and force conditions on the diaphragm (left image) and tool shaft (right image).

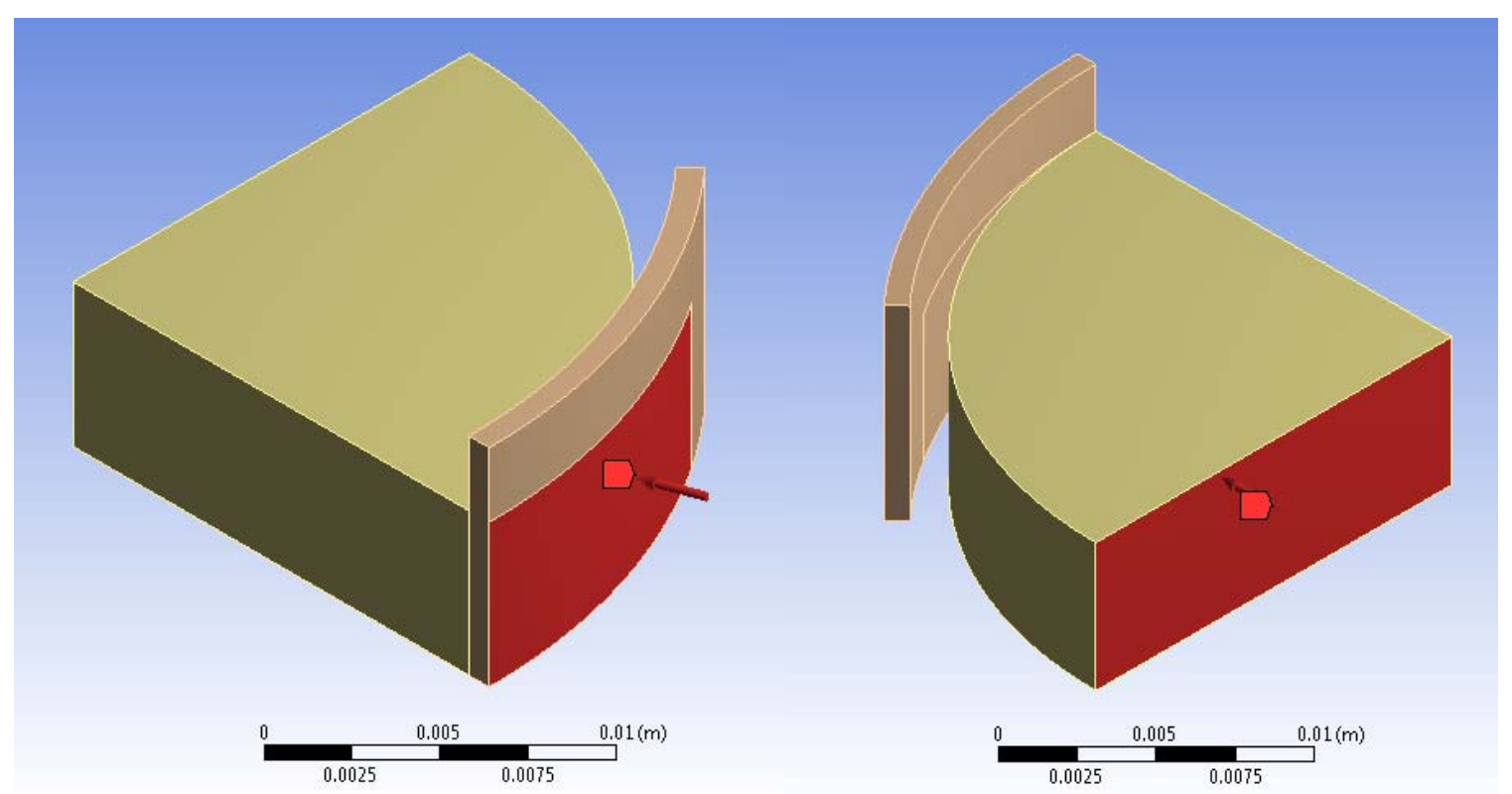

Figure 4.14 - Pressure and force conditions on model 
The analysis was completed using large deflections which are common practice for a non-linear hyperelastic material analysis. An implicit solver was used for linear convergence of the nonlinear solution. The total deformation of the model was solved for using a static structural analysis in ANSYS Workbench. The displacement of the shaft as interpreted from the simulation, represented by $d_{\text {simulation }}$, must be considered with respect to the actual center position of the shaft to determine the displacement of the shaft from the center position represented by $r_{u}$. See Figure 4.15 for a diagram of the center position of the shaft and the displacement of the shaft from simulation results.

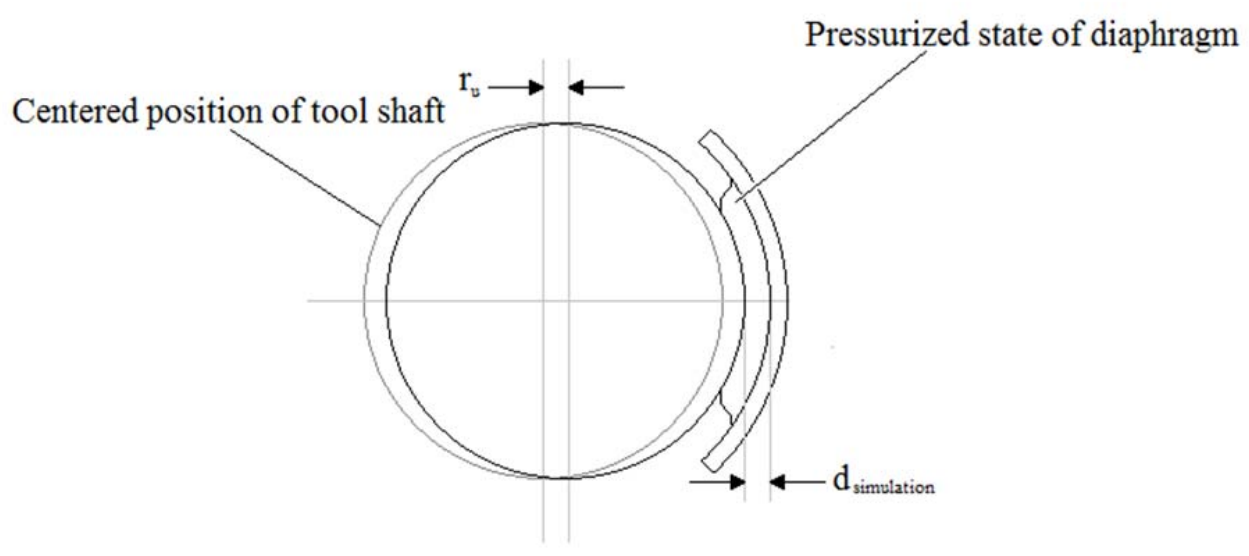

Figure 4.15 - Position of tool shaft with respect to diaphragm

See Figure 4.16 below for a diagram of the results of $1 \mathrm{~N}$ of shaft force and $200 \mathrm{kPa}$ of pressure on the diaphragm. This is one case required to determine the behaviour of the actuator. The maximum total deformation is the displacement of the shaft $d_{\text {simulation }}$. However, depending on the force and pressure applied to the system the diaphragm may deform more than the shaft. In these cases the $d_{\text {simulation }}$ will be the maximum shaft body displacement. See Appendix A for the complete ANSYS simulation results for total deformation of the model. 


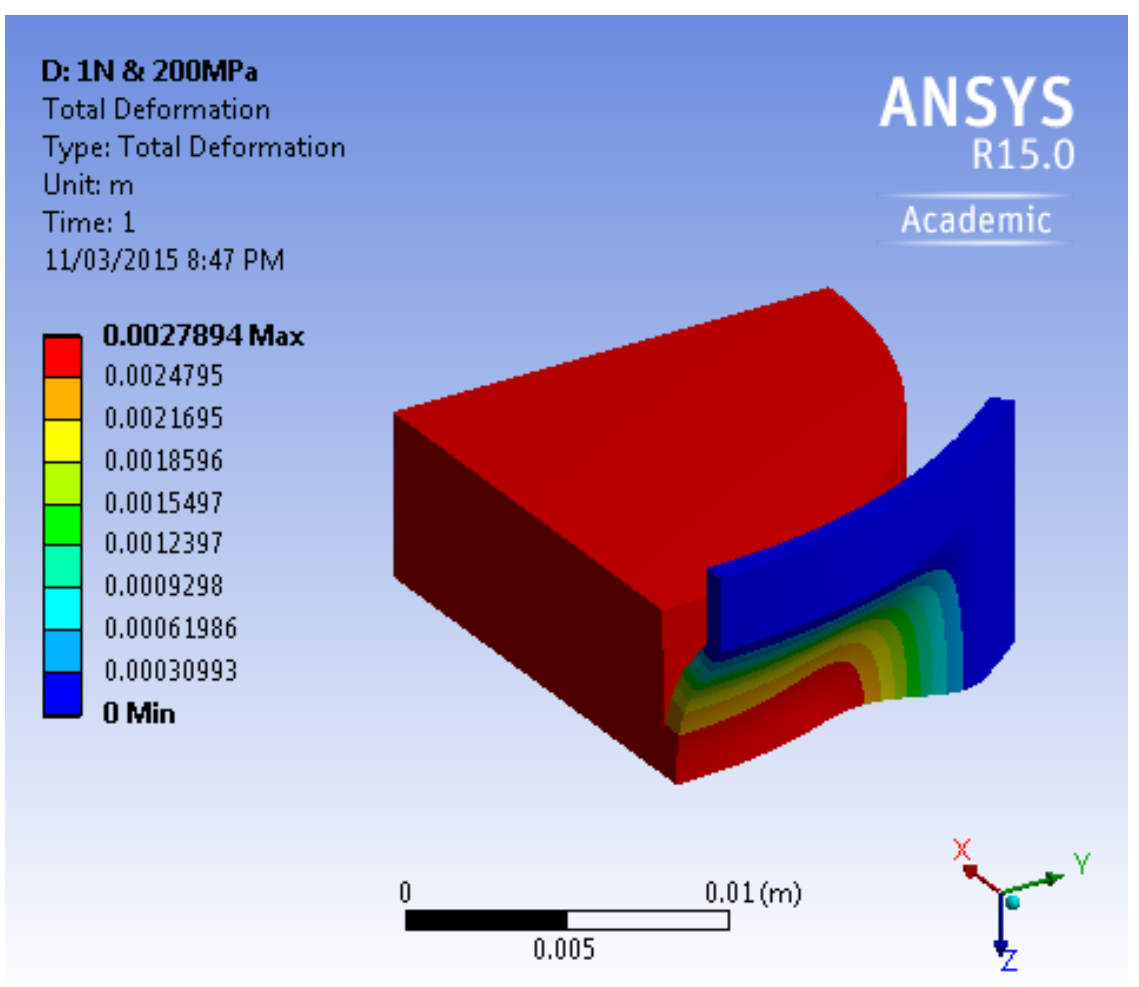

Figure 4.16 - Total displacement of ANSYS finite element model

The pressure on the diaphragm and force on the shaft were varied within the design requirements to determine a stiffness relationship with the pressure of the diaphragm. The force on the tool tip was varied between $5.50-16.49 \mathrm{~N}$, and the pressure was varied between $100-400 \mathrm{kPa}$. The radial displacement of the tool shaft from the centered position was calculated by subtracting $d_{\text {simulation }}$ and the radial thickness of the tool shaft from the total radial distance of the diaphragm. The resulting displacement was used to determine the stiffness of the tool based on the force on the tool shaft. The stiffness values were plotted by against pressure for relationships based on tool shaft force. See Figure 4.17 for a plot of stiffness vs. pressure simulation results. The plots were curved fitted to exponential functions as they appear to exhibit an exponential trend. 


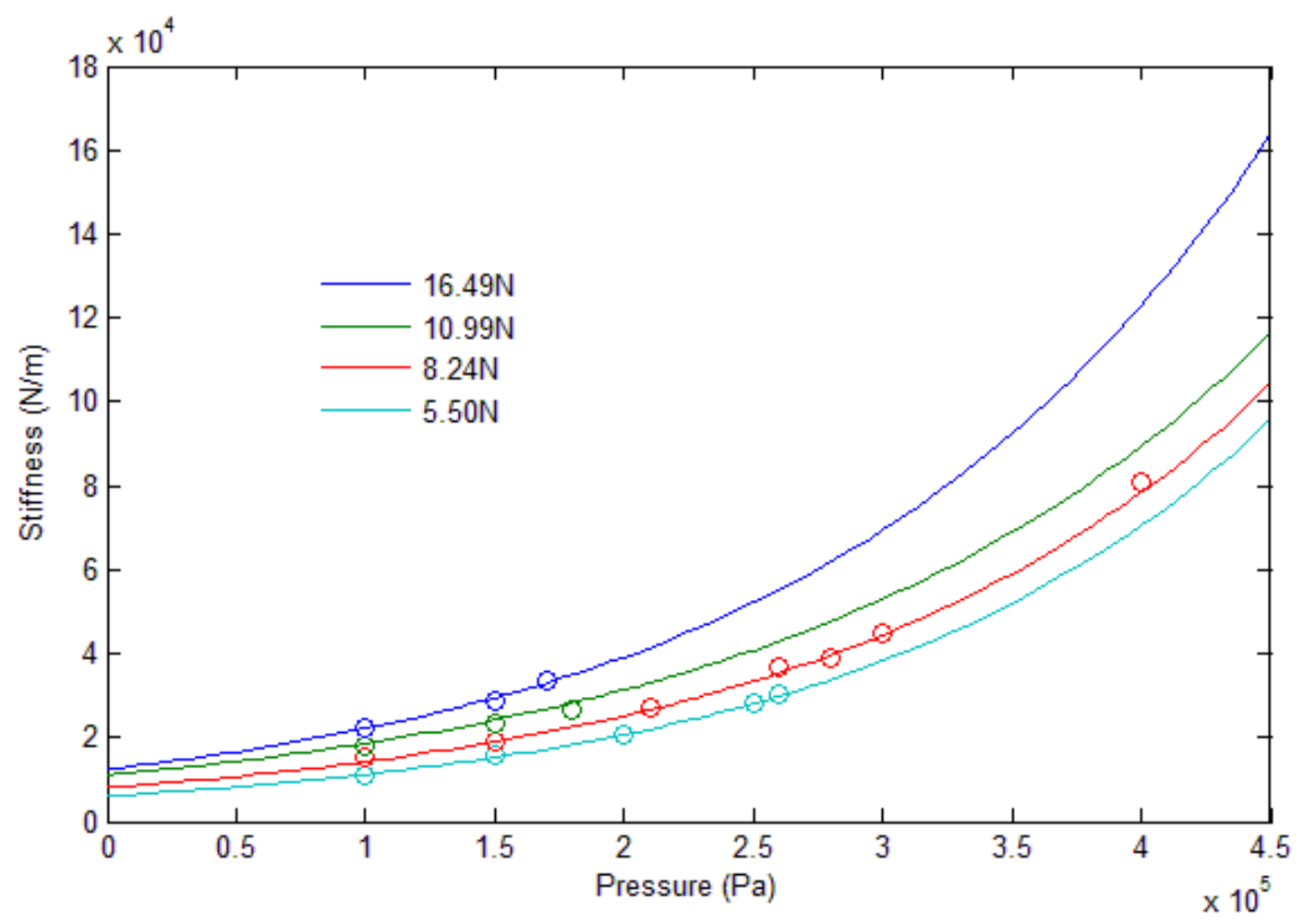

Figure 4.17 - Stiffness pressure simulation results 


\section{Chapter 5}

\section{Fabrication and Testing}

Fabricating and testing the ERA is a difficult and tedious effort. Fabrication of the ERA must be greatly improved compared to the PRA as this was one its key areas that required improvement and affected its performance. Testing the ERA is important to precisely determine the calibration relationship of the sensor and tool dynamics as well to determine the stiffness behaviour of the tool accurately. This section will develop the fabrication and assembly methods used to create the pneumatic actuator and related testing equipment.

\subsection{Fabrication}

The ERA was fabricated through NC machining of Aluminum 6061 billets. Parts were manufactured by Viking Engineering \& Tool. Two dimensional drawings were produced via three dimensional mockups using CATIA V5. The ERA assembly consists of the main housing, four diaphragm retainers, the silicone rubber diaphragm, and 32 machine screws. See Figure 5.1 for a picture of the final ERA assembly. The assembly procedure was integrated into the design of the tool. Tooling was created along with the actuator assembly. The tooling consists of a Cclamp, an outer clamping block, and an inner clamping block. The inner clamping block is designed such that the diaphragm retainer can be clamped to the housing and the outer clamping block is designed such that the housing can be clamped to the diaphragm retainer. The diaphragm sits between the housing and the retainer. 


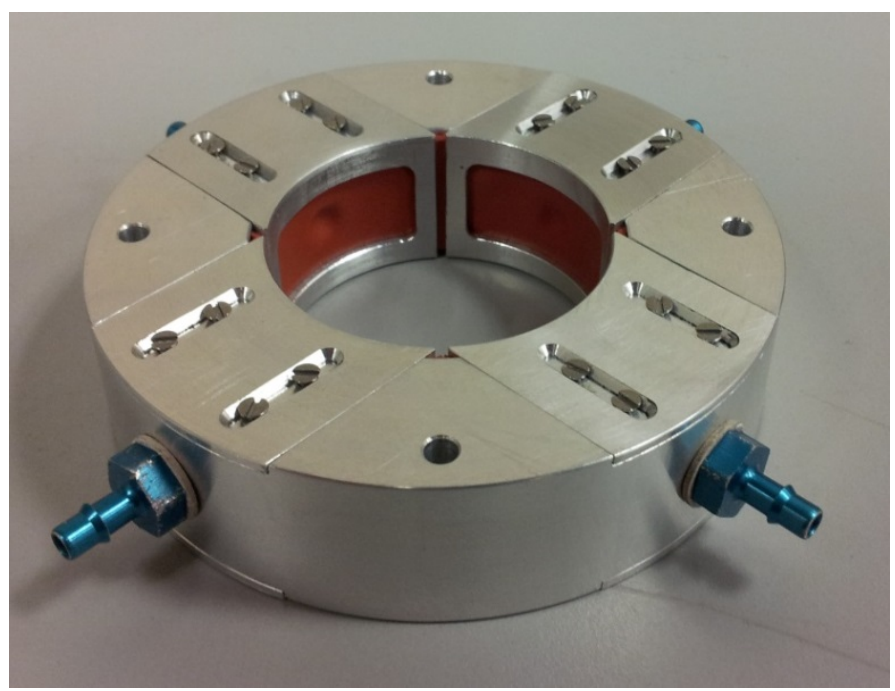

Figure 5.1 - Enhanced Radial Actuator

The actuator was designed to be assembled in this way in order to create a clamping condition on the diaphragm along the surface of the retainer to which it contacts. The retainer was designed to maintain this clamping pressure with eight machine screws. The machine screws were selected for their dimension as they were required to sit flush with the actuator surface. The bevel for the screw slot could also not penetrate the retainer thickness and in order to reduce shear stress on the screws and prevent deformation the retainer must have an unbeveled depth of $0.005 \mathrm{in}$. In addition the screws must be required to maintain the clamping force on the retainer. See Figure 5.2 for a diagram of the assembly process for clamping the diaphragm with the diaphragm retainer. 


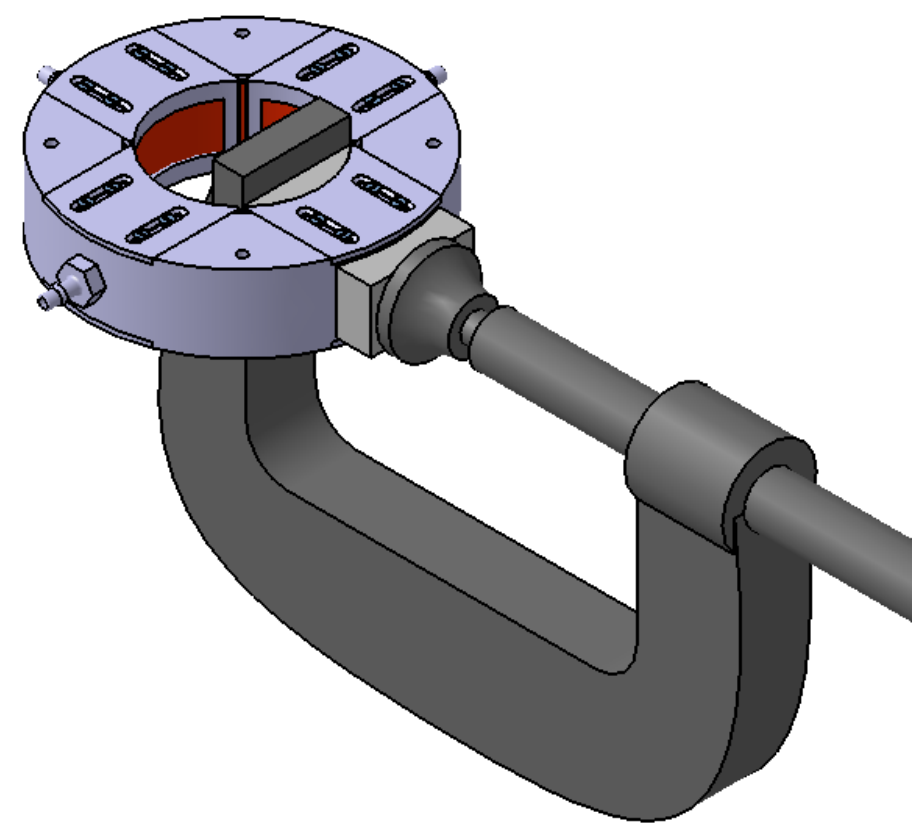

Figure 5.2 - Assembly of the enhanced radial actuator

\subsection{Calibration}

The tool has the ability to sense the radial displacement of the tool tip through rotation of the gimbal. The displacement of the shaft is tracked using two magnets, fixed to the shaft, which lie in close proximity to two Honeywell HMC1501 magnetic displacement sensors fixed to the tool's housing. The displacement of these magnets will result in a change in the position of the magnetic field lines, through which the sensor detects and translates into a voltage. The relationship of the displacement of the tool tip and the respective voltage must be established to use the sensor to determine tool tip position. This relationship was determined by Petz in his work for a different magnet [12]. The relationship was determined holding the magnet close to the sensor in approximately in the position where the magnet would be placed in the tool. See Figure 5.3 for previous calibration setup. 


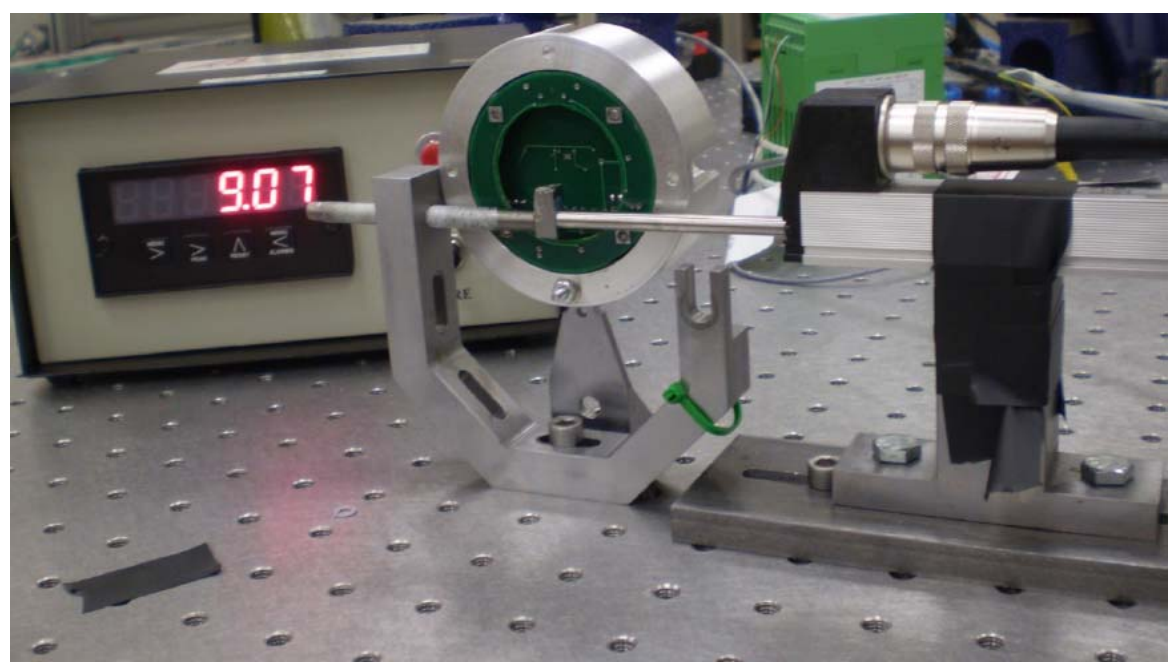

Figure 5.3 - Calibration of the magnetic sensor [12]

This method was effective to determine the relationship between displacement of the magnet used and the voltage output of the sensor.

A practical demonstration of the voltage and displacement relationship should be established to accurately track the tool's position. The magnet previously used was a larger magnet that was cut which may affect the magnetic fields of each magnet. Cutting the magnet may affect the uniformity of the field lines with respect to the way they are mounted on the tool shaft. To address this, two 0.25 in $\times 0.25$ in $\times 0.125$ in Neodymium magnets were acquired and positioned so that their north poles faced the magnetic sensor. The $\mathrm{x}$ and $\mathrm{y}$ sensors share a whetstone bridge that may have a cross-talk effect on sensor output. This can effectively change the sensor displacement and voltage relationship. This can be addressed by calibrating the sensor in situ with the deburring tool fully assembled.

The tool was mounted to a table vice which was mounted to a rigid testing platform. Three calibration configurations were attempted. In Figure 5.4 two concepts can be seen, each using a dial indicator to measure the displacement of the tool tip. In the left image the dial indicator was preloaded to give the tool tip appropriate displacement. The tool tip was also held using the small spring stiffness of the dial indicator tip and the tip of the threaded rod. In the image to the right 
the setup was slightly improved by placing the tool tip within a cup to hold it to travel with the threaded rod. These still yielded inaccurate results as the tool tip wandered over the axes to which it was not being actuated. It was clear that the tool must only be allowed to move within a prescribed degree of freedom to accurately calibrate it.
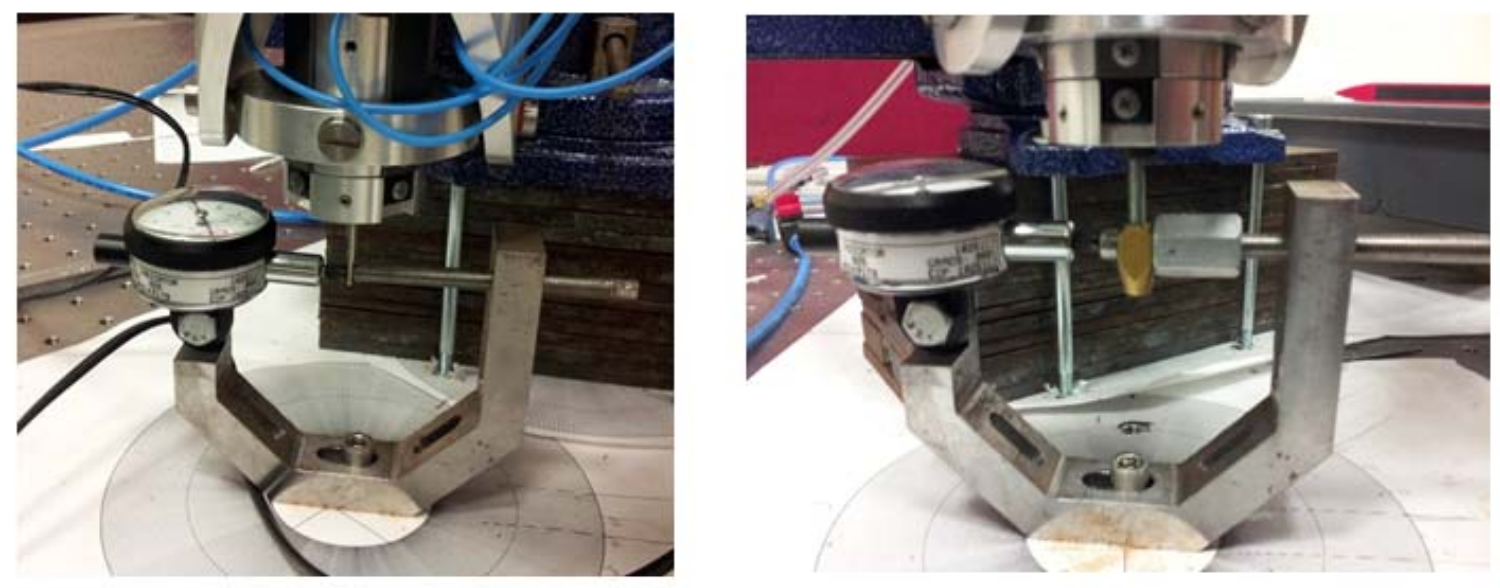

Figure 5.4 - Two concepts for calibration rigging

A new calibration setup was designed to achieve this, see Figure 5.5 for a concept model of the calibration stage. The use of a two axis stage which was actuated by two micrometers to accurately determine the displacement in two degrees of freedom. The deburring tool tip was attached to the stage via a close tolerance ball joint to which the shaft of the tool tip could freely slide within. 


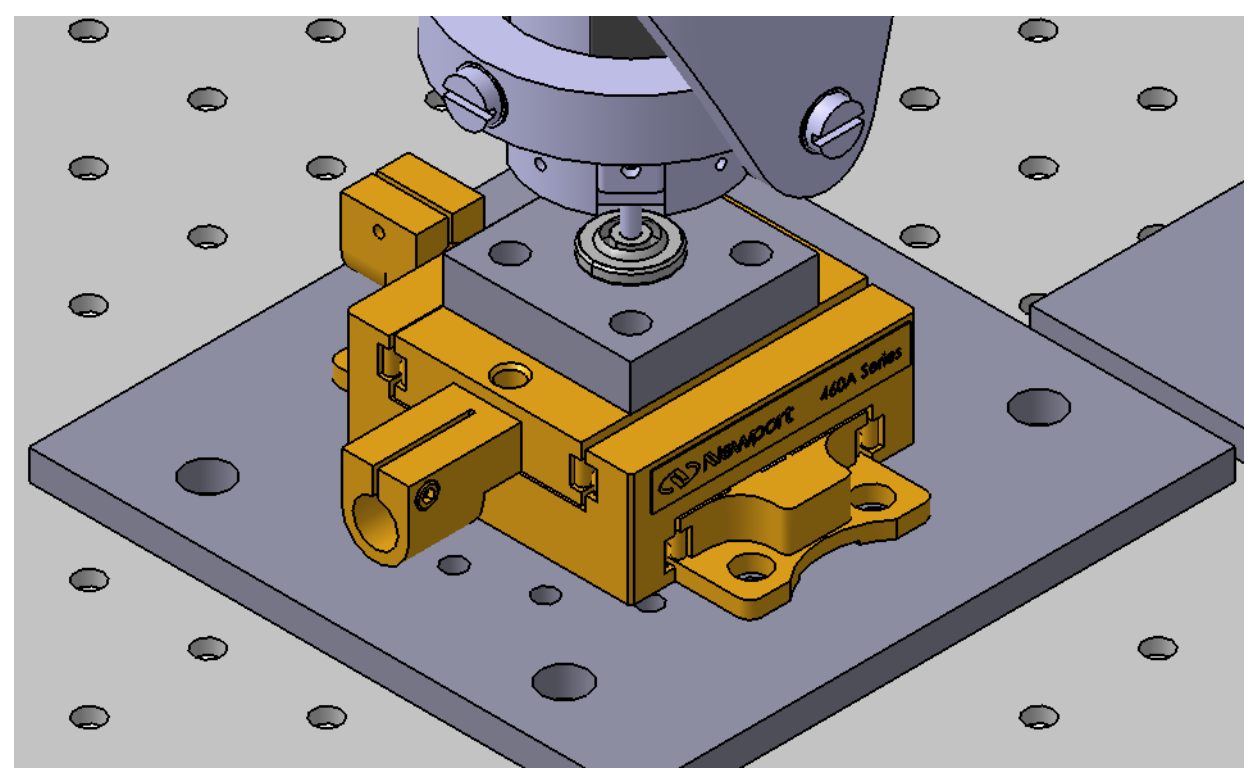

Figure 5.5-Calibration setup concept

The final calibration stage was created using adapter plates that linked each component together to the common fixed rigid testing platform. See Figure 5.6 for an image of the final calibration stage setup.

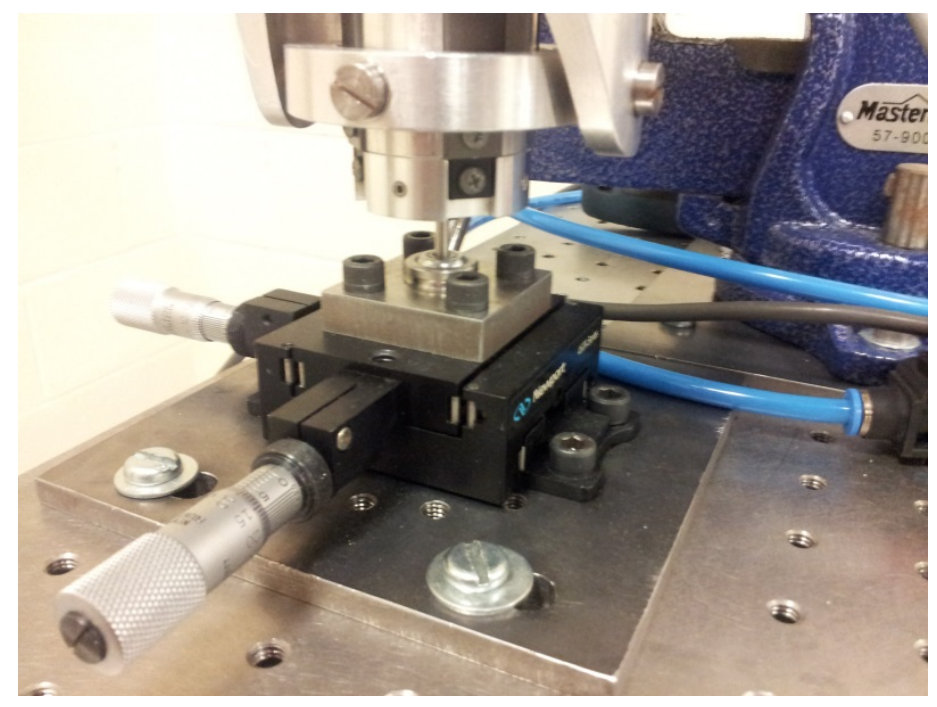

Figure 5.6 - Final calibration rigging

Calibration was completed on the two axis stage from Newport with manual micrometers also from Newport. The custom board for the magnetic field sensors and surround architecture can be found in the appendices. Data was collected through a Measurement Computing USB 
data acquisition unit at 100samples per second. Signal handling was accomplished using Matlab Simulink. In Figure 5.7 the first system represents the data acquisition device with two signals; channel 0 representing the $\mathrm{x}$-axis sensor and channel 1 representing the $\mathrm{y}$-axis sensor. The subsystem outputs both $\mathrm{x}$ and $\mathrm{y}$ voltage signals to the result $\mathrm{XY}$ scope. In the subsystem below is where the individual bias is applied to each signal.
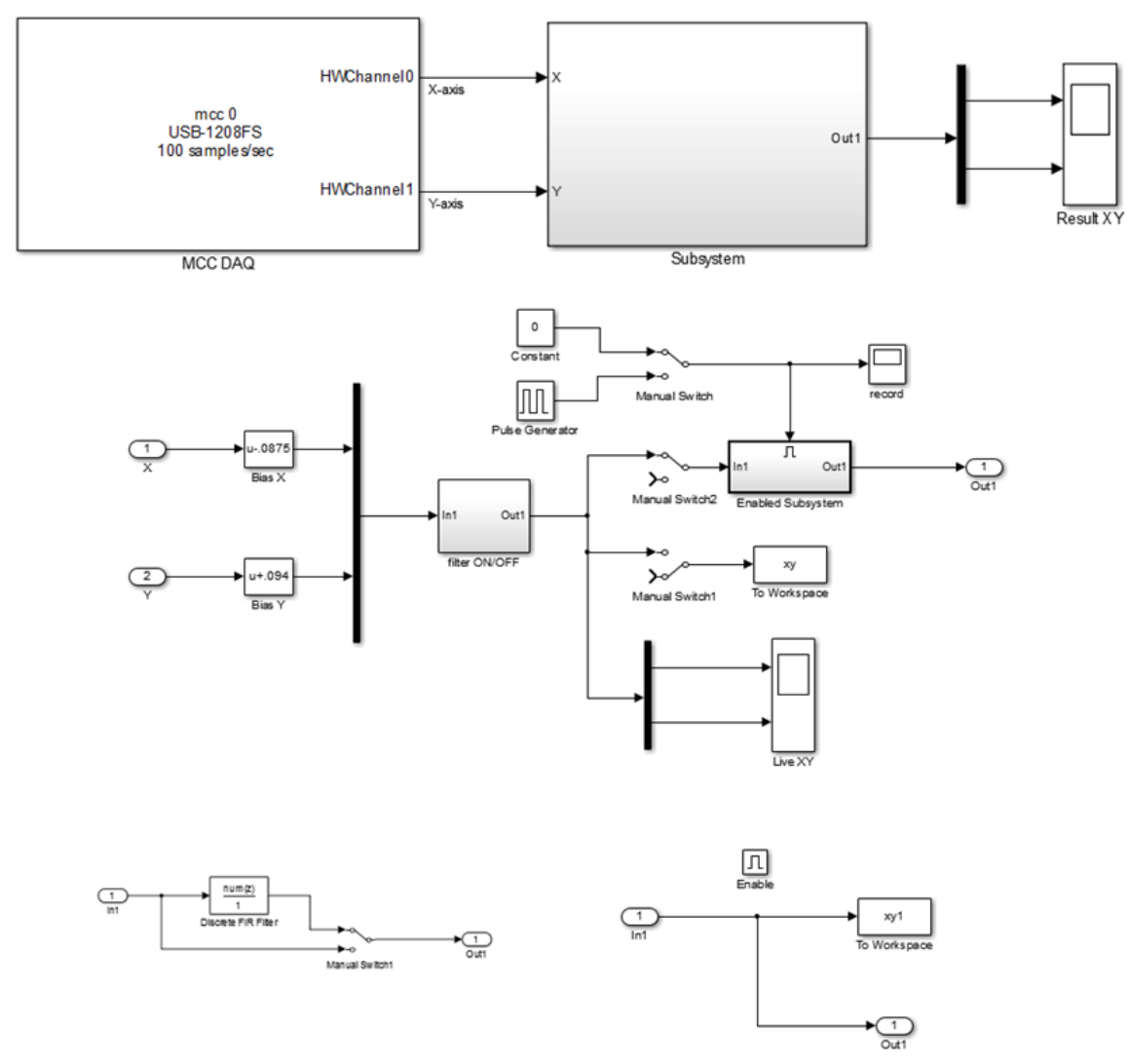

Figure 5.7 - Simulink Program for Calibration

A bias was determined for each sensor using a removable plastic collar which fit within the deburring tool between the inner shaft of the tool and the outer casing as can be seen in Figure 5.8. The collar will hold the tool shaft directly in the center to determine a bias. The bias is present due to tolerance stack-ups, magnetic cross-talk, and imperfections in the magnet placement. Once the shaft was centered and the bias determined it could be slid downward and out of the way of the tool shaft's envelope of movement. 


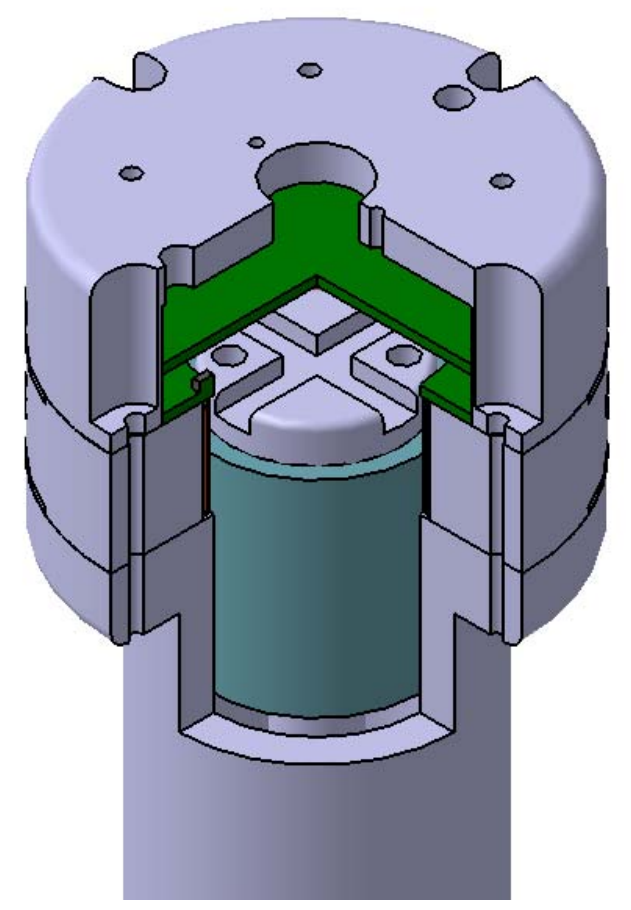

Figure 5.8-Centering Collar (light blue)

A filter was applied to reduce the noise within the signals. The calibration data was a combination of measurements from the micrometers and signals recorded from the data acquisition unit. In order to make recording and processing the data as simple as possible, a pulse generator was use to record exactly one value of the voltage for both $\mathrm{x}$ and $\mathrm{y}$ sensors. This allowed time for the micrometers to be set before taking another recording. The result is a series of voltage records which would correspond to manually recorded position records. The calibration resulted in a linear relationship for each sensor. See Figure 5.9 for the plot of voltage vs. displacement and equation (5.1) and (5.2) for the formula for relationship. To verify the accuracy of the voltage and displacement relationship the sensor was calibrated at 22.5 degree increments 360 degrees around the tool. 


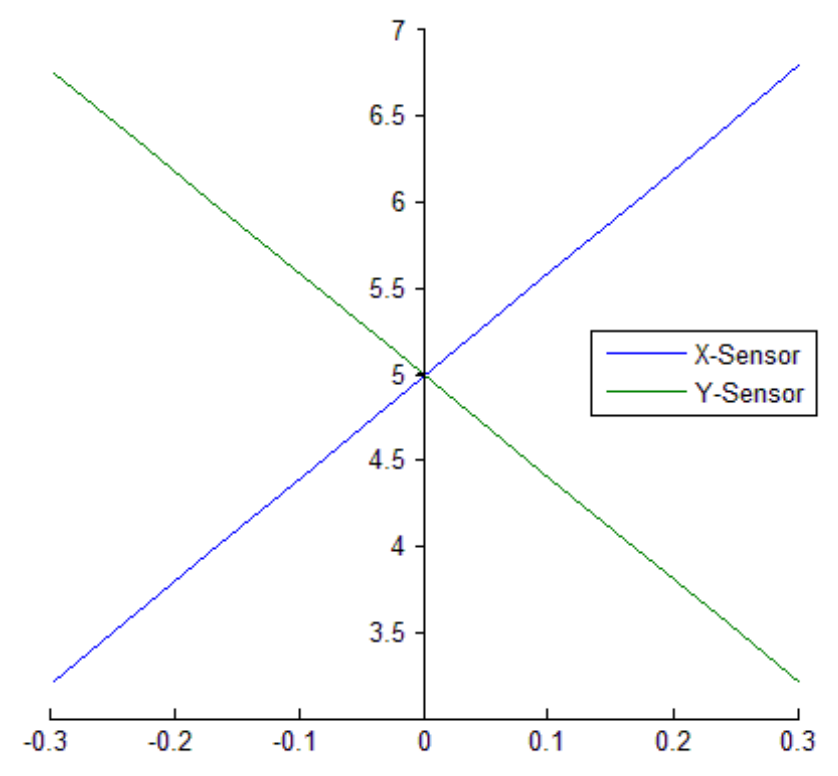

Figure 5.9 - Voltage vs. Displacement Relationship

$$
\begin{gathered}
x=5.922 V_{x \text { sensor }}+5.0682 \\
y=-5.9129 V_{y \text { sensor }}+5.0377
\end{gathered}
$$

These relationships can be used to convert the signal from each sensor into accurate displacement data for further testing. Since calibration has been completed it is not possible to determine the dimensional resolution of the $\mathrm{x}$ and $\mathrm{y}$ sensors. Since the maximum resolution for the sensors based on the data acquisition is $2.28516 \mathrm{mV}$. This value can be multiplied by the slope of each sensor voltage and displacement relationship. This would provide the $\mathrm{x}$ and $\mathrm{y}$ sensors with $0.0135 \mathrm{~mm}$ of resolution to displacements of the tool shaft in the radial direction. The requirement for displacement resolution was less than $0.1 \mathrm{~mm}$ and the tool's achievable resolution is almost ten times the requirement.

\subsection{Testing}

Once calibrated, the tool could be tested for its stiffness behaviour. This would be accomplished by loading tool tip and varying the pressure of the ERA. Using the calibrated sensor the position in the $\mathrm{x}$ and $\mathrm{y}$ directions could be recorded. Data was recorded using the same data acquisition 
unit. An Omega PX319 pressure transducer was used to accurately measure the pressure on the ERA over the duration of data recording. The displacement was measured using the magnetic sensors and converted using the voltage / displacement relationship determined through calibration. Weights were hung from a fishing line fixed to the tool tip and left to hang off the rigid testing table, see Figure 5.10 for an image of the experimental setup. The mass of each weight was measured and recorded for each test. The tool was pressurized and data recorded. The pressure was varied within the ERA and held for small time increments in order to create clear displacement and pressure data sets. Data was recorded continuously and sorted for stable pressure and displacement values.

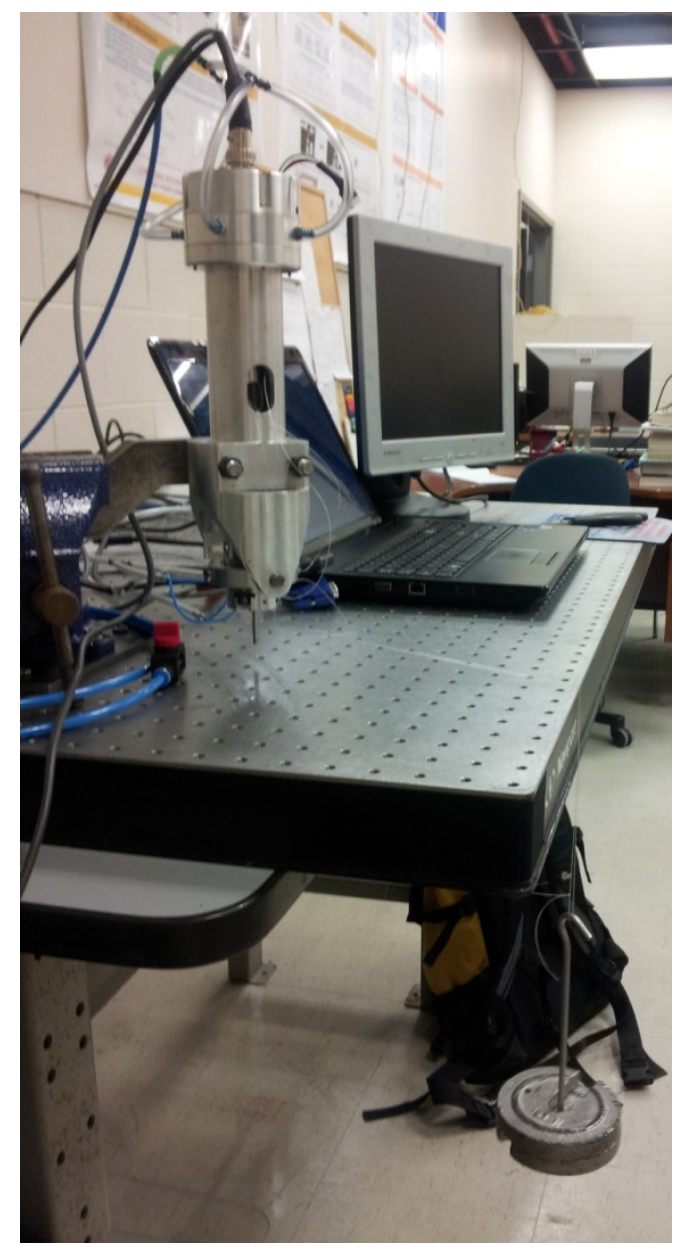

Figure 5.10 - Stiffness Testing Setup 
The stiffness was determined based on the force on the tool tip and the displacement caused as a result of the force and pressure setting. As the pressure was varied for a constant tool tip force a noticeable change of displacement and therefore stiffness was observed. The stiffness and pressure relationships were plotted as can be seen in Figure 5.11. The plots were curve fitted and like the simulation results they too appeared to correlate to exponential functions.

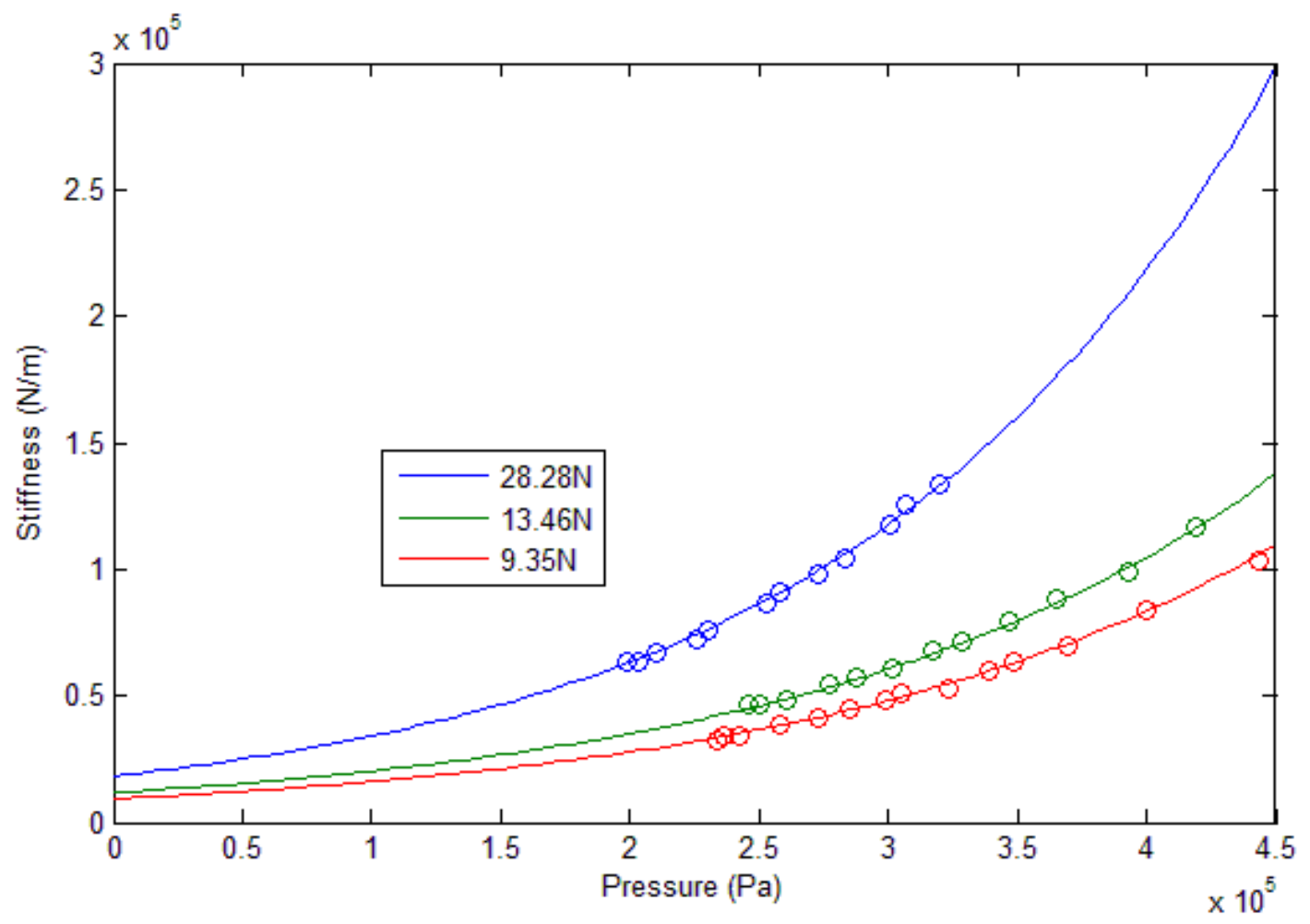

Figure 5.11 - Experimental stiffness and pressure testing results

Exponential stiffness curves were determined for experimental testing on the ERA. These curves can be compared to the exponential curves created from simulation data. See Figure 5.12 for a comparison of experimental data to simulation data. 


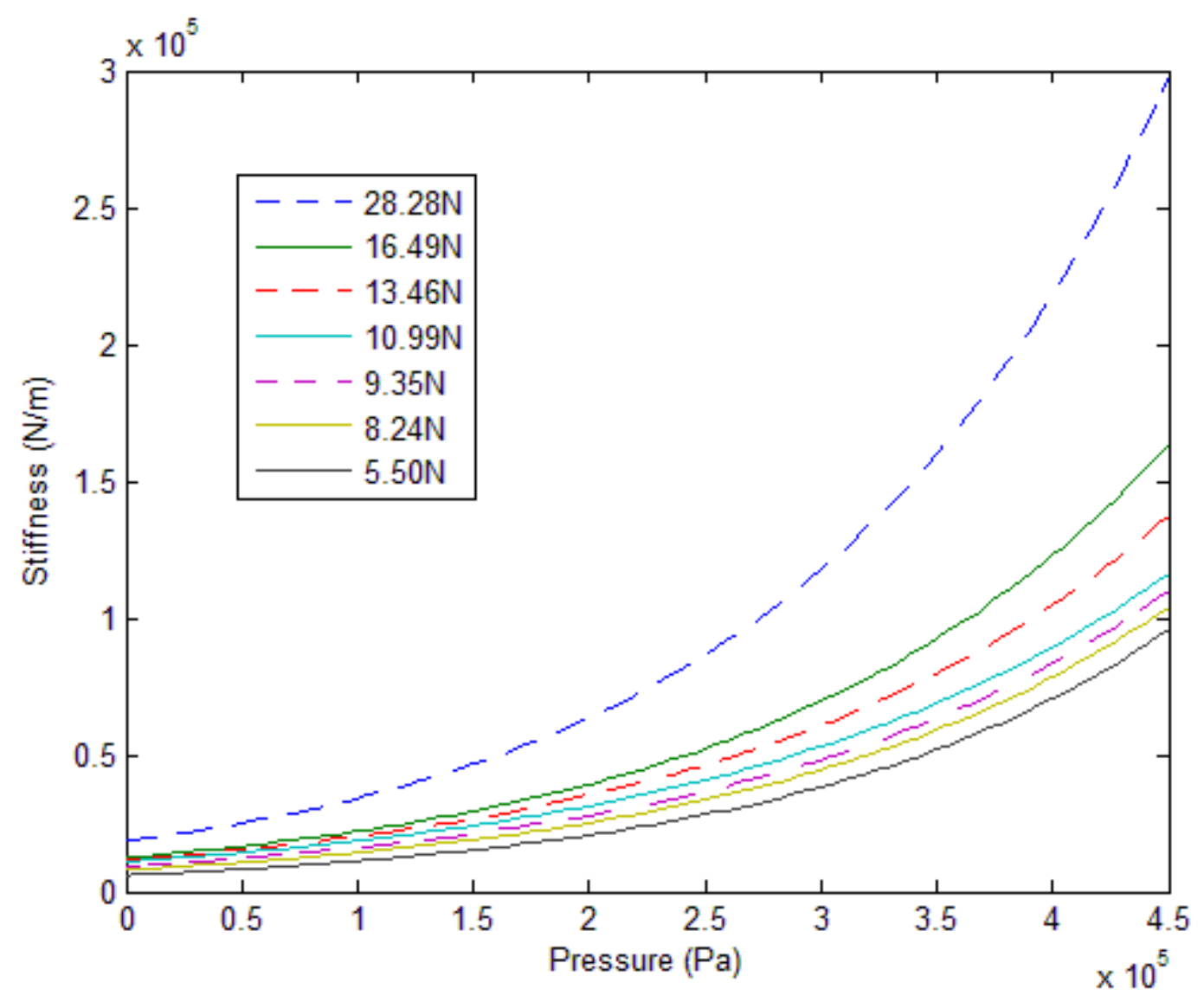

Figure 5.12 - Stiffness vs. pressure relationship for simulation and testing data

Testing data curves are represented with hatched lines, whereas simulation data is represented with solid lines. Comparing curves from the simulation to the curves from experimental testing it is clear that they follow the same relationship. Both fall within a logical trend that appears to increase as the shaft force increases. Exponential functions follow the form of equation (5.3).

$$
K_{r}=a_{\text {exp }} e^{b_{\text {exp }} p}
$$

The radial actuation stiffness is represented by $K_{r}$ and is a function of pressure and two exponential coefficients $a_{\text {exp }}$ and $b_{\text {exp }}$. These coefficients are independently a function of tool tip loading. Figure 5.13 is a plot of the first exponential coefficient as a function of tool tip loading. Both simulation and testing data points follow the same increasing relationship that 
appears to follow a linear relationship. Figure 5.14 is a plot of the second exponential coefficient as a function of the tool tip loading. These values seems to follow a piecewise linear relationship that initially decreases and then changes direction to increase for increasing tool tip loading.

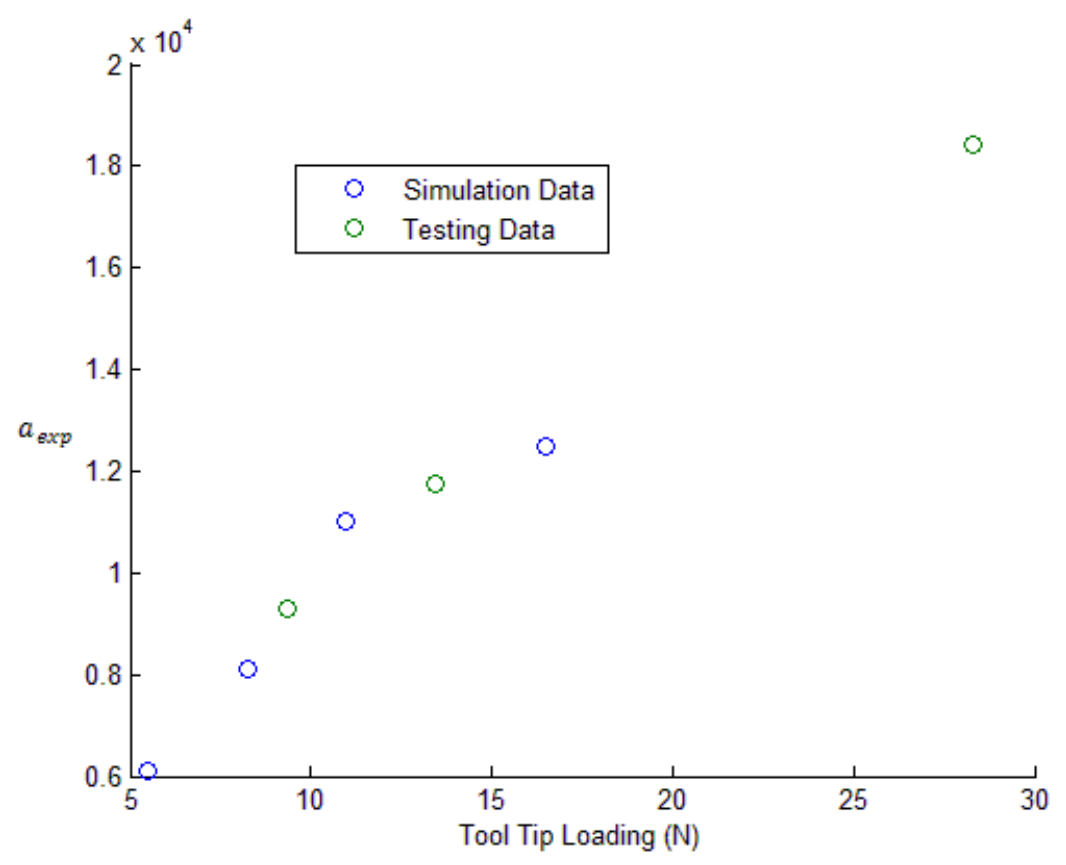

Figure 5.13 - $a_{\text {exp }}$ vs. Tool Tip Loading

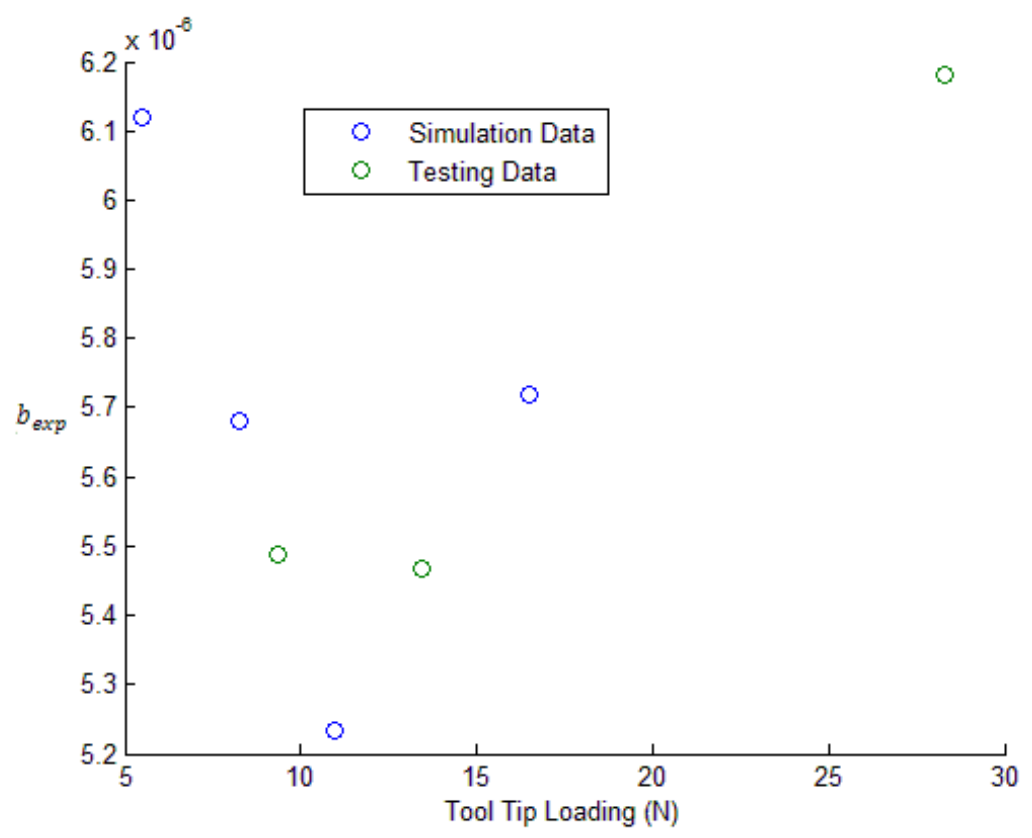

Figure 5.14 - $b_{\text {exp }}$ vs. Tool Tip Loading 
The stiffness behaviour of the ERA must be compared to the stiffness behaviour of the PRA to determine if an improvement has been made. See Figure 5.15 for the stiffness vs. pressure plot of the Petz PRA and the ERA.

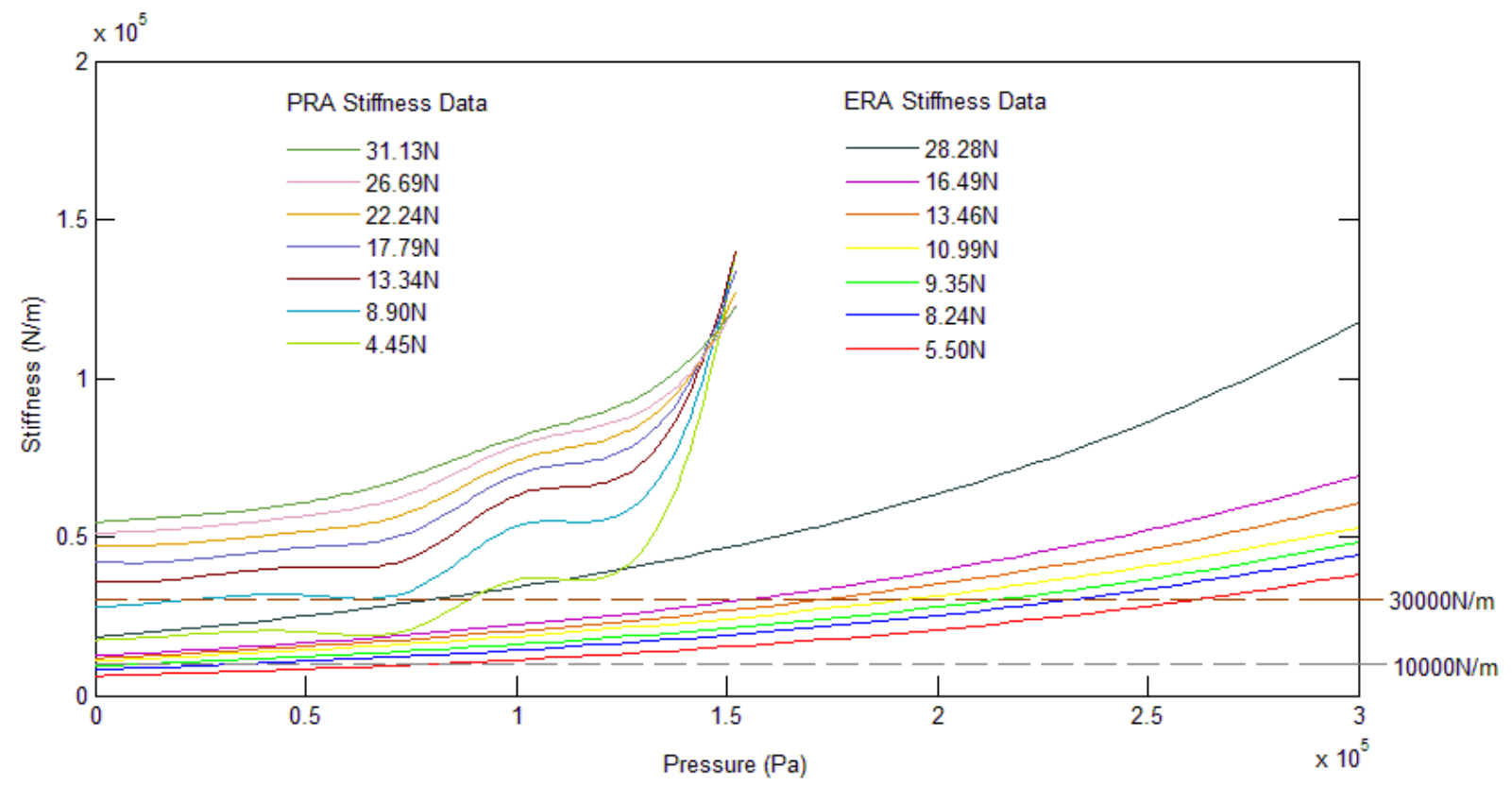

Figure 5.15 - Stiffness behaviour between the PRA and ERA

When comparing the stiffness relationship of the PRA with the ERA it is apparent that the two behave very differently. The PRA stiffness was measurable with no positive pressure differential in the rubber chamber. When not inflated the PRA will have a stiffness relationship. As the rubber material is stretched into position this will cause inherent stiffness in the tool shaft. This can alter the tool's ability to be in a centered position throughout its operating pressure range. When the ERA is not inflated there is no stiffness in the tool shaft allowing the tool shaft to be free. When inflated the ERA will symmetrically inflate all four diaphragms to contact the shaft and maintain a centered position. This will allow for a greater degree of flexibility for compliance in the tool shaft as there is not a minimum stiffness achievable on tool shaft. Figure 5.15 indicates this as for the ERA at $5.50 \mathrm{~N}$ the stiffness is lower than the PRA minimum 
stiffness for $4.45 \mathrm{~N}$. If a stiffness of zero was required this may be achieved by pressurizing the ERA just enough to make contact with the tool shaft but not provide stiffness.

The two actuators appear to have different stiffness ranges and the relationships over this range. This is important to identify a superior design as the range of stiffness required is defined in as a requirement in section 3.1. The PRA does not perform within the stiffness range required; 10000 to $30000 \mathrm{~N} / \mathrm{m}$. The ERA does perform within the design requirements as can be seen by the dashed lines in Figure 5.15. The PRA does not seem to have a simple relationship between the pressure and stiffness, which would be a polynomial of an order greater than six. This would be very difficult to determine a relationship as for a given tool tip load as it could have multiple pressure values. The ERA however, follows a natural logarithm. The natural logarithm will only have a single stiffness value for each tool tip load and pressure. This would be significantly easier to determine a relationship for control purposes as it is only dependent on variation of two constants. 


\section{Chapter 6}

\section{Conclusion and Future Work}

Throughout the research presented the requirement for an active compliant deburring tool has been enumerated. The work completed has conveyed how the ERA can achieve this need as it has been successfully improved upon previous work and designs as contributed to the development. Future work is required to complete a fully automated solution to precision deburring applications. This section will summarize the work presented and academic contributions as well as it will provide future considerations.

\subsection{Conclusion}

In this thesis report it was determined an automated precision deburring process was required. To fulfill this need, compliance is required to be at the core of the design. To meet this requirement a system must be proposed that can achieve compliance in three degrees of freedom. To achieve compliance in three degrees of freedom an action plane was created to simplify the actuation of the system into radial and axial directions. It was determined that the Petz HFCDT was an excellent platform to improve upon as it was designed to have axial and radial actuation using principles of compliance to remove burrs. However, the PRA has problems of unsymmetrical stiffness characteristics and difficult to manufacture, thereby requiring further development before being used in a precision deburring application.

It was determined that the radial actuation of the tool must be redesigned to achieve the original compliance goals. A design process was initiated and out of eight concepts a final design was selected. The ERA designed used four silicone rubber diaphragms arranged in a ring shape facing inwards to act upon the free rotating tool shaft. The free tool shaft ensures that compliance is possible leaving it up to the diaphragms to provide stiffness to the system. The diaphragms 
were simulated using a finite element model analysis. Silicone rubber is a non-linear material and was modelled using an Arruda-Boyce hyperelastic constitutive model. The deformation of the diaphragm was modelled for various pressures and tool shaft forces. The data was formed into stiffness vs. pressure plots and curve fitted to exponential functions. The simulation of the radial actuator produced positive results as the stiffness trend appeared to follow increasing exponential functions as the tool shaft force and pressure increased.

The ERA was fabricated and assembled as designed with little to no difficultly. The diaphragm performed as expected when compared to the simulation results. The radial position sensor required calibration. A calibration rig was designed using a two axis translational stage and micrometers to measure displacement. The sensors were calibrated and linear relationships were determined based on the voltage output and displacement of magnets fixed to the tool shaft. The stiffness behaviour of the tool had to be determined; a series of tests were created using weights to load the tool tip while the pressure was varied within the ERA, and displacement values were recorded. The stiffness values were plotted and curve fitted to exponential functions. The exponential functions were compared to those that were obtained through simulation and it was determined that the functions followed the same increasing trend as was seen in the simulation results. When compared to the PRA's stiffness characteristics it was clear that an exponential trend was present only in the ERA's stiffness behaviour. The PRA's stiffness behaviour did not seem to present a stiffness behaviour that was easily predictable. By comparing the stiffness requirements to the exponential curves for the ERA, it was clear that it met these requirements. The stiffness increases as the pressure increases, and the stiffness required for increasing tool tip loading increases as well. 


\subsection{Contributions}

The lack of commercially available solutions for precision deburring has left the industry to continue to use hand deburring techniques. Many solutions have been proposed to fulfill the industry need but none have come closer than Petz's HFCDT. The tool however has a critical flaw that must be solved in order to continue its journey into commercial use. The radial actuation required significant improvement before it could be further tested on for its ability to complete burr removal in a precision application. The solution created is the ERA.

- The radial actuator's displacement was successfully predicted using a finite element model analysis. The model used the non-linear hyperelastic material model known as the eight chain or Arruda-Boyce model to describe the behaviour of the silicone rubber. The model was tested for a range of pressure and tool tip loading conditions. The data was curve fitted and used to define the stiffness behaviour.

- The design for the ERA was successfully manufactured using simple manufacturing techniques. The radial actuator qualitatively performs as expected with no malfunction or unexpected performance issues.

- The magnetic sensors were successfully calibrated and functioned in situ as designed. The $\mathrm{x}$ and y displacement relationship between the voltage output was determined, and correctly returned the position of the tool tip through the use of an analog to digital USB data acquisition device. The sensors achieved a radial displacement resolution of $0.0135 \mathrm{~mm}$, almost ten times the minimum requirement.

- The radial stiffness of the tool was tested and compared to the simulation stiffness relationships. A correlation was determined to suggest that the radial stiffness of the tool could be predicted based on the simulations preformed. The tool met the required stiffness range of 10,000 to $30,000 \mathrm{~N} / \mathrm{m}$. 
- The radial stiffness of the tool was compared to the radial stiffness of the previous PRA, and it was determined that there was a significant improvement in the behaviour. Over the range of the tool tip loading conditions the ERA performed with more stability than that of the PRA. This was evident from the predictable trend discovered from ERA's stiffness plots.

\subsection{Future Work}

Several aspects of the tool's design could be improved for future work on the subject. The tool's main shaft could be redesigned for positional precision during assembly. Currently the rotating shaft of the tool is made up of two casings A and B. To assemble them they fit together through high tolerance cylindrical fit, fixed with two set screws. Casing A is precisely fixed to the gimbal axes and will fit together in the same manner every time it is assembled. Casing B can rotate 360 degrees and will not necessarily fit the same way. This is however a problem as the magnet which is used to calibrate the magnetic sensors could be slightly altered if assembled incorrectly after calibration. To overcome this, the shaft mating surfaces should be keyed alignment of the magnets with respect to the magnetic sensors.

The radial position sensor should be digitized so it does not rely on analog to digital data acquisition past the sensor housing. This will minimize noise in the signal and inconsistency of results. The tool will require testing with on-line burr removal and edge breaking. Future work would include non-linear pressure control of the radial and axial actuators. The nonlinear control would be based on their non-linear stiffness behaviour. A controller would be required to achieve this, which would be significant work in the commercialization of the deburring tool. 


\section{Bibliography}

[1] R. K. Roberts, T. W. Engel and F. Proctor, "Specification of an active force control tool for performing deburring and chamfering on a robot platform," Industrial Electronics, Control, Instrumentation, and Automation, IEEE, San Diego, pp. 918-926, 1992.

[2] F. Xi, Advanced Aerospace Manufacturing, AE8141 Lecture notes, Ryerson University, Toronto, 2008.

[3] L. K. Gillespie and P. T. Blotter, "The formation and properties of machining burrs," Journal of Manufacturing Science and Engineering, vol. 98, no. 1, pp. 66-74, 1976.

[4] B. Kennedy, Decisive Deburring, Cutting Tool Engineering, pp. 28-35, 2005.

[5] J. M. Schimmels, "Design of an admittance control law for precision edge tracking in robotic deburring," Advances in Robotics, Mechatronics, and Haptic Interfaces, vol. 49 pp. 281-286, 1993.

[6] J. M. Schimmels, "Multidirectional compliance and constraint for improved robotic deburring," Robotics \& Computer Integrated Manufacturing, vol. 17, no. 4, pp. 277-294, 2001.

[7] L. Liao, J. Xi and K. Liu, "Modeling and control of automated polishing/deburring process using a dual-purpose compliant toolhead," International Journal of Machine Tools \& Manufacture, vol. 48, pp. 1454-1463, 2008.

[8] B. M. Kramer and S. S. Shim, "Development of a system for robotic deburring," Robotics \& Computer-Integrated Manufacturing, vol. 7, no. 3-4, pp. 291-295, 1990.

[9] M. G. Her and H. Kazerooni, "Automated Robotic Deburring of Parts Using Compliance Control," Journal of Dynamic Systems, Measurement, and Control, vol. 113, pp. 60-66, 1991.

[10] Available: www.ati-ia.com/products/deburr/deburring_home.aspx. [Accessed 15 Decemeber 2014].

[11] L. Liao, Modelling and Control of Automated Polishing/Deburring Process, Ph.D. Thesis, Ryerson University, Toronto, 2008. 
[12] B. A. Petz, Design, Analysis and Testing of a Radial-Axial Hybrid Active Force Compliant Tool Head for Deburring Turbine Engine Components, Master's Thesis, Ryerson University, Toronto, 2011.

[13] K. Stouffer, J. Michaloski, B. Russell and F. Proctor, "ADACS - An automated system for part finishing," in Industrial Electronics, Control, and Instrumentation, (Washington, DC), 1993.

[14] K. Stouffer, R. Russell, R. Archacki, T. Engel, R. Dansereau and A. Grot, "Advanced Deburring and Chamfering System (ADACS) Final Report," National Institute of Standards and Technology, 1996.

[15] J. H. Chung and C. Kim, "Modeling and control of a new robotic deburring system," Robotica, vol. 24, no. 2, pp. 229-237, 2006.

[16] C. Grosjean, G. B. Lee, W. Hong, W. Tai and C. M. Ho, "Micro balloon actuators for aerodynamic control," in Eleventh Annual International Workshop on Micro Electro Mechanical Systems, pp. 166-171, Heidelberg, 1998.

[17] X. Yang, C. Grosjean, Y.-C. Tai and C.-M. Ho, "A MEMS thermopneumatic silicone membrane valve," in The Tenth Annual International Workshop on Micro Electro Mechanical Systems an Investigation Of Micro Structures, Sensors, Actuators, Machines and Robots, Nagoya, 1997.

[18] R. M. Tomastik, A. Enomoto and T. W. Engel, "Concept for Robotic Deburring Using Multipass Active Control," Journal of Vibration and Control, vol. 3, no. 3, pp. 351-369, 1997.

[19] A. d. Bever, Dynamic Behavior of Rubber and Rubberlike Materials, Report, Eindhoven University of Technology, Eindhoven, 1992.

[20] Available: http://aerospace.honeywell.com/ /media/Images/Plymouth\%20Website\%20PDFs/Magnetic\%20Sensors/Data\%20Sheets/HMC1501-1512.ashx. [Accessed 13 December 2014].

[21] H. Thomas and V. Hoersch, Stresses Due to the Pressure of One Elastic Solid Upon Another, Report, University of Illinois, Urbana, 1930. 
[22] E. M. Arruda and M. C. Boyce, "A three dimensional constitutive model for the large stretch behavior of rubber elastic materials," Journal of the Mechanics and Physics of Solids, vol. 41, no. 2, pp. 389-412, 1993.

[23] H. Zheng, On the Predictive Capability and Stability of Rubber Material Models, Master's Thesis, Massachusetts Institute of Technology, Cambridge, 2008.

[24] Available: http://www.meanwell.com/search/DR-30/DR-30-spec.pdf. [Accessed 15 December 2014].

[25] Available: http://www.omega.com/pptst/PX309-5V.html. [Accessed 15 December 2014].

[26] Available: www.mccdaq.com/pdfs/manuals/USB-1208FS.pdf. [Accessed 15 December 2015]. 


\section{Appendices}

\section{Appendix A ANSYS Total Deformation Results}

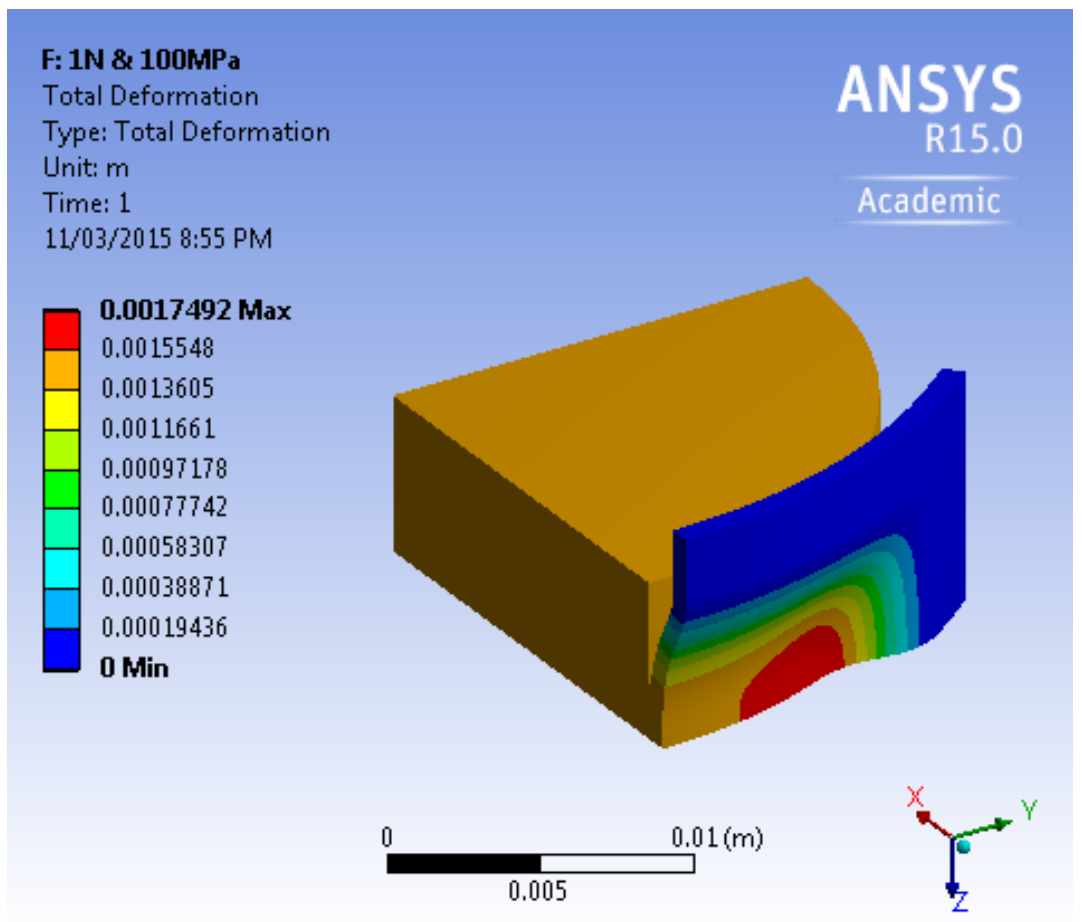

Figure A-1 Results for $1 \mathrm{~N}$ shaft force and $100 \mathrm{MPa}$ diaphragm pressure

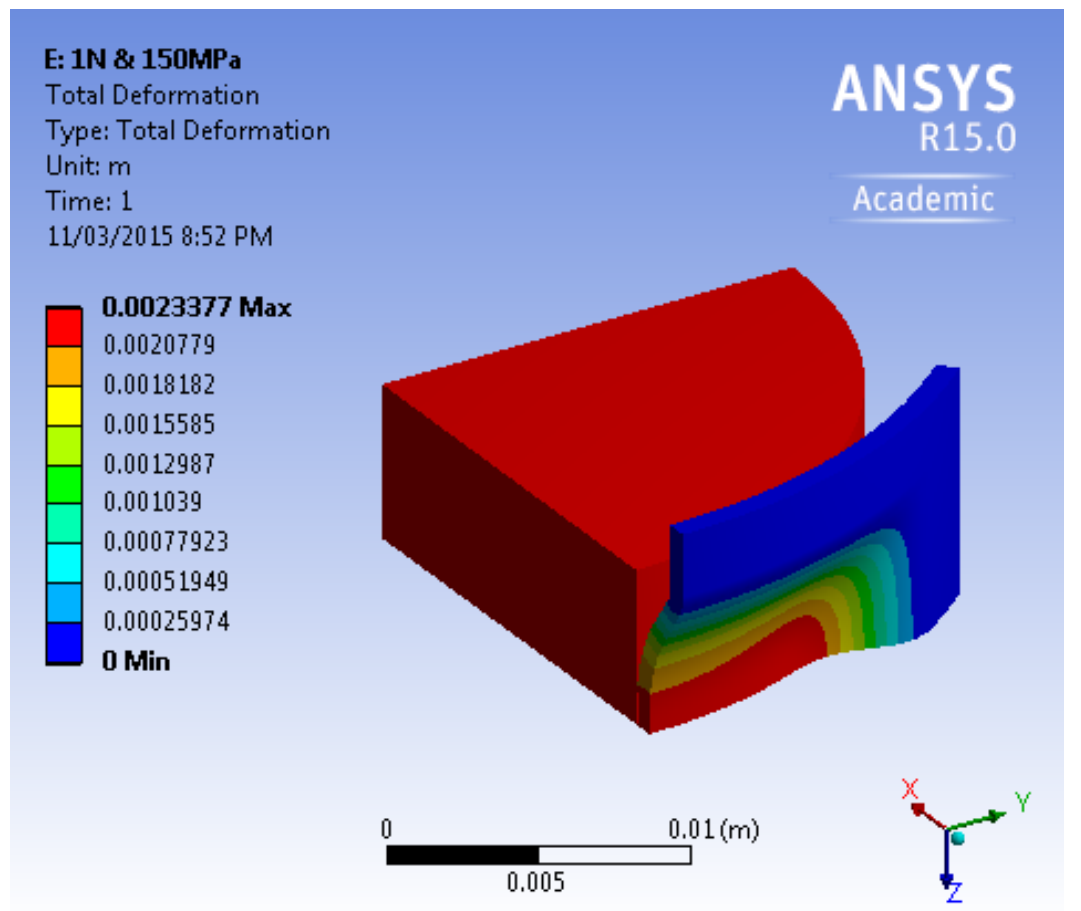

Figure A-2 Results for $1 \mathrm{~N}$ shaft force and $150 \mathrm{MPa}$ diaphragm pressure 


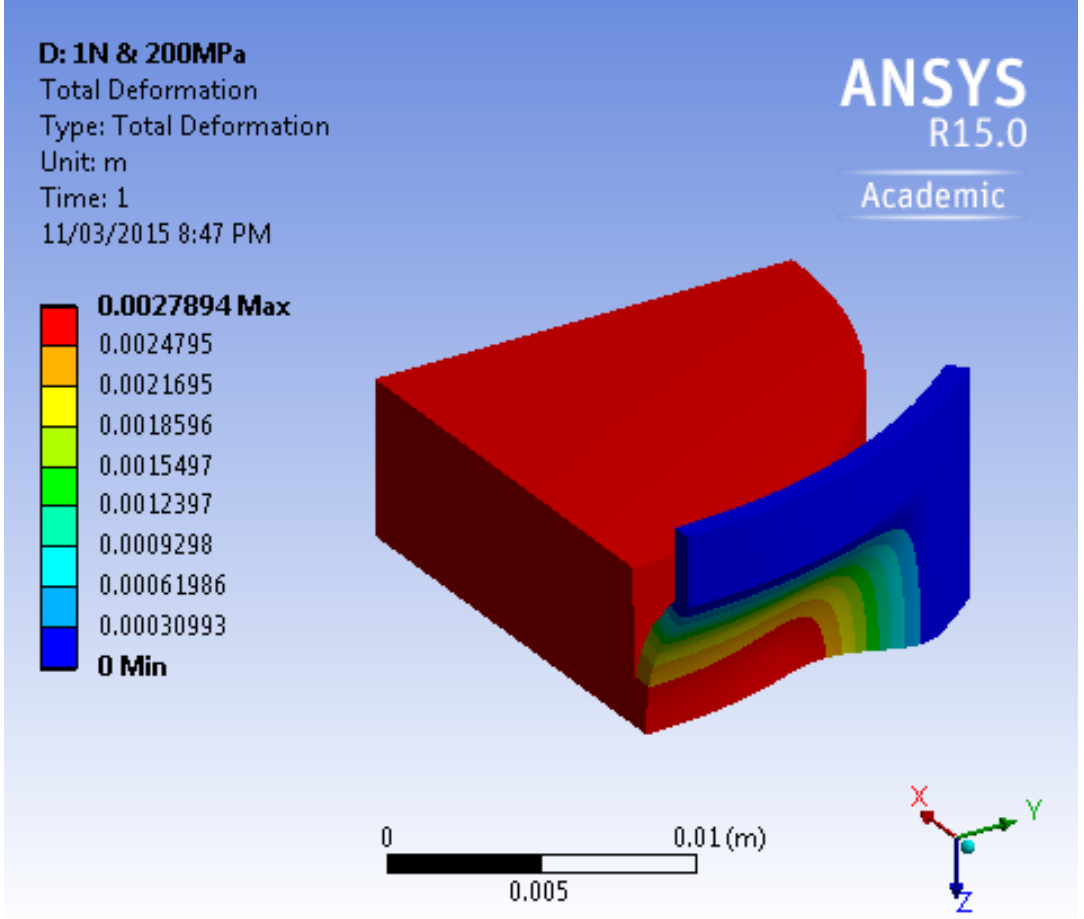

Figure A-3 Results for $1 \mathrm{~N}$ shaft force and 200MPa diaphragm pressure

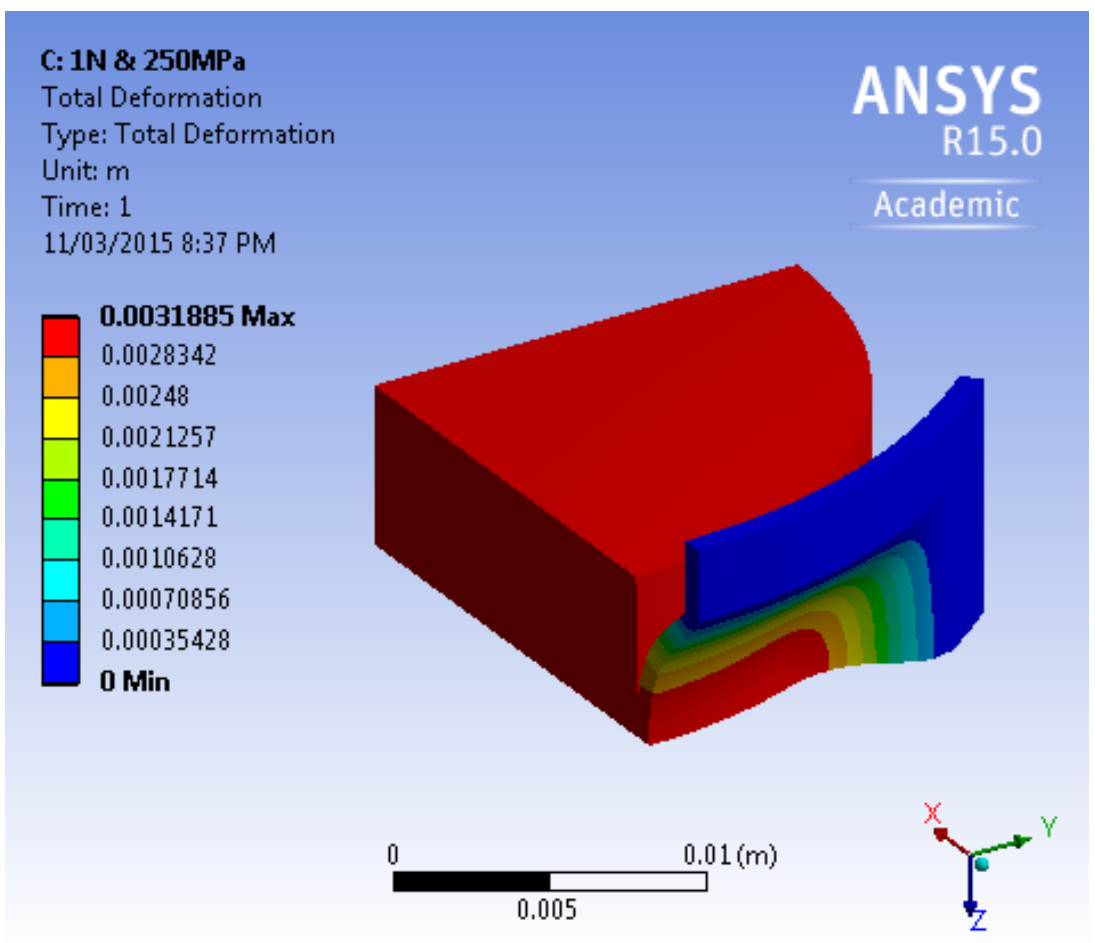

Figure A-4 Results for $1 \mathrm{~N}$ shaft force and $250 \mathrm{MPa}$ diaphragm pressure 


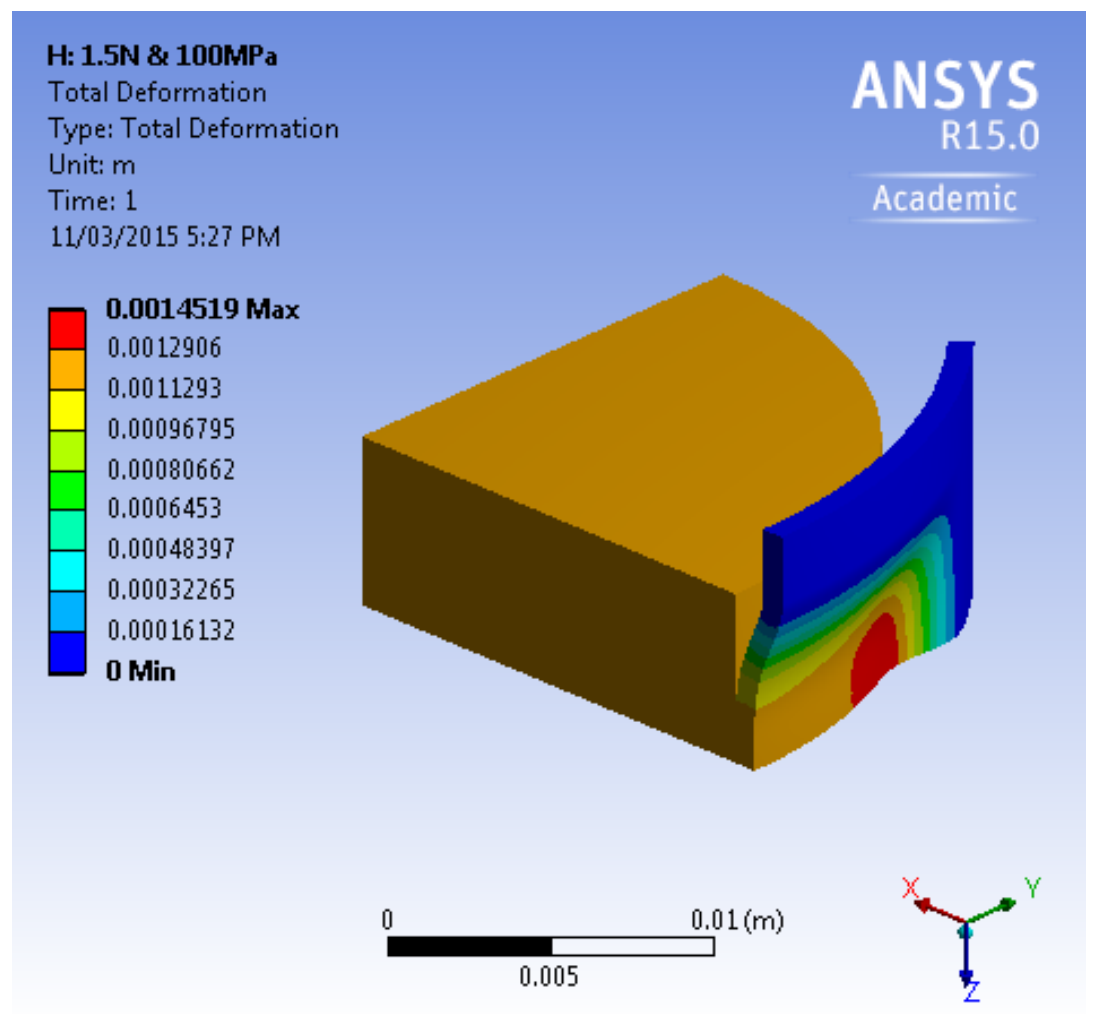

Figure A-5 Results for $1.5 \mathrm{~N}$ shaft force and 100MPa diaphragm pressure

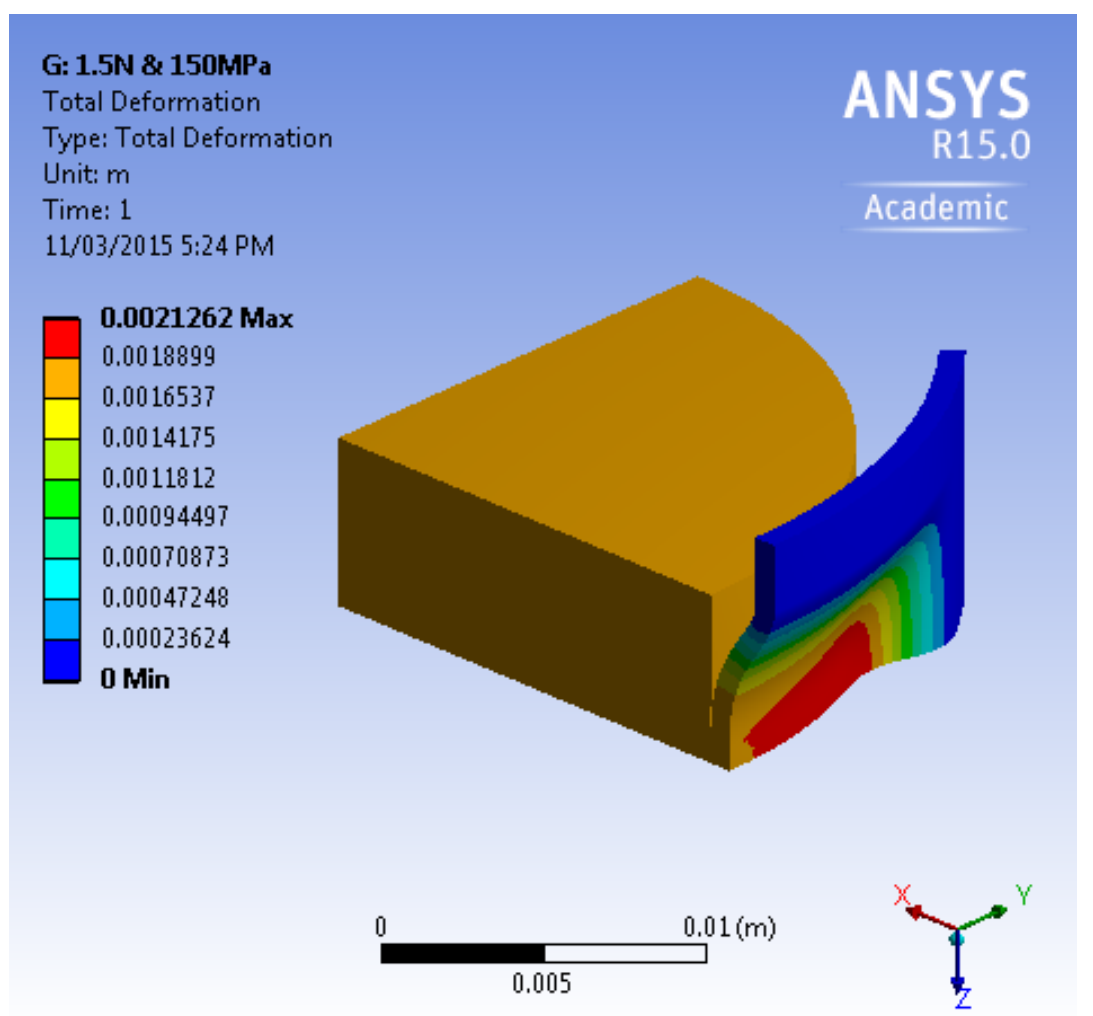

Figure A-6 Results for $1.5 \mathrm{~N}$ shaft force and $150 \mathrm{MPa}$ diaphragm pressure 


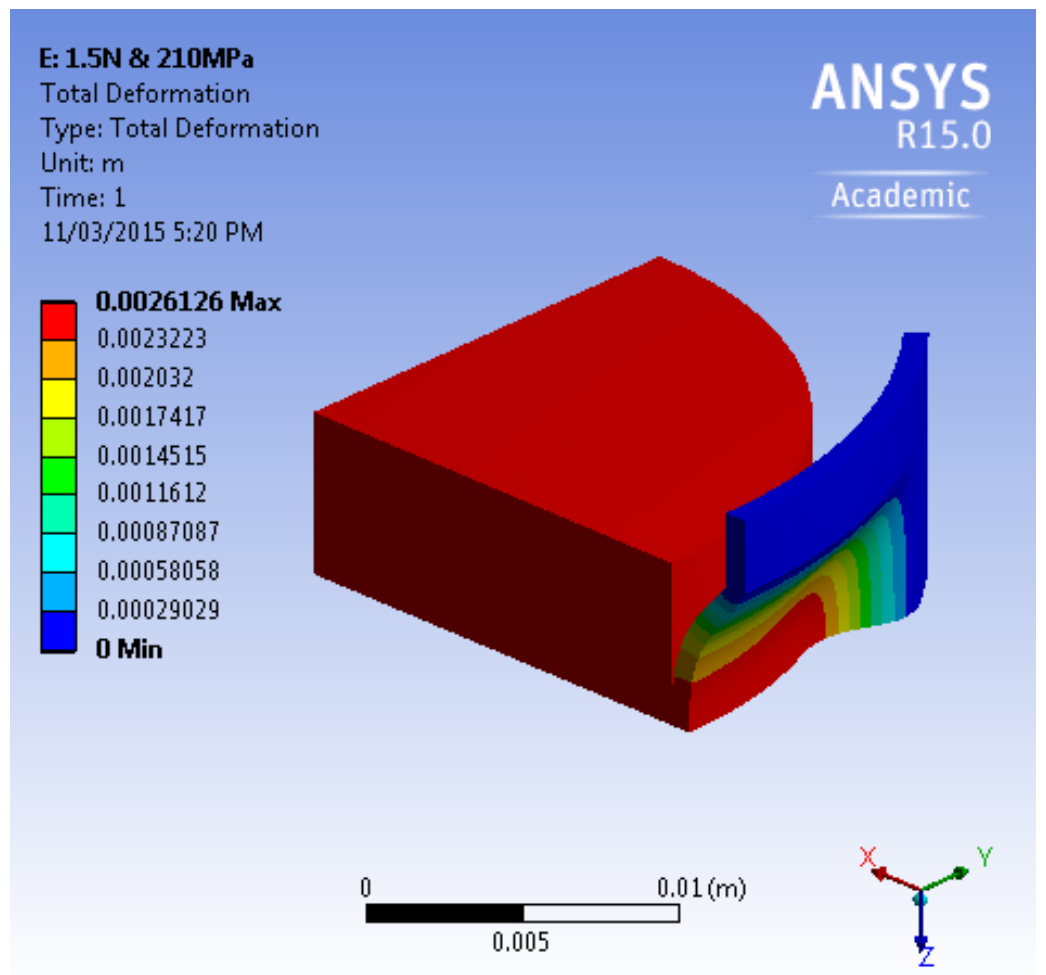

Figure A-7 Results for $1.5 \mathrm{~N}$ shaft force and $210 \mathrm{MPa}$ diaphragm pressure

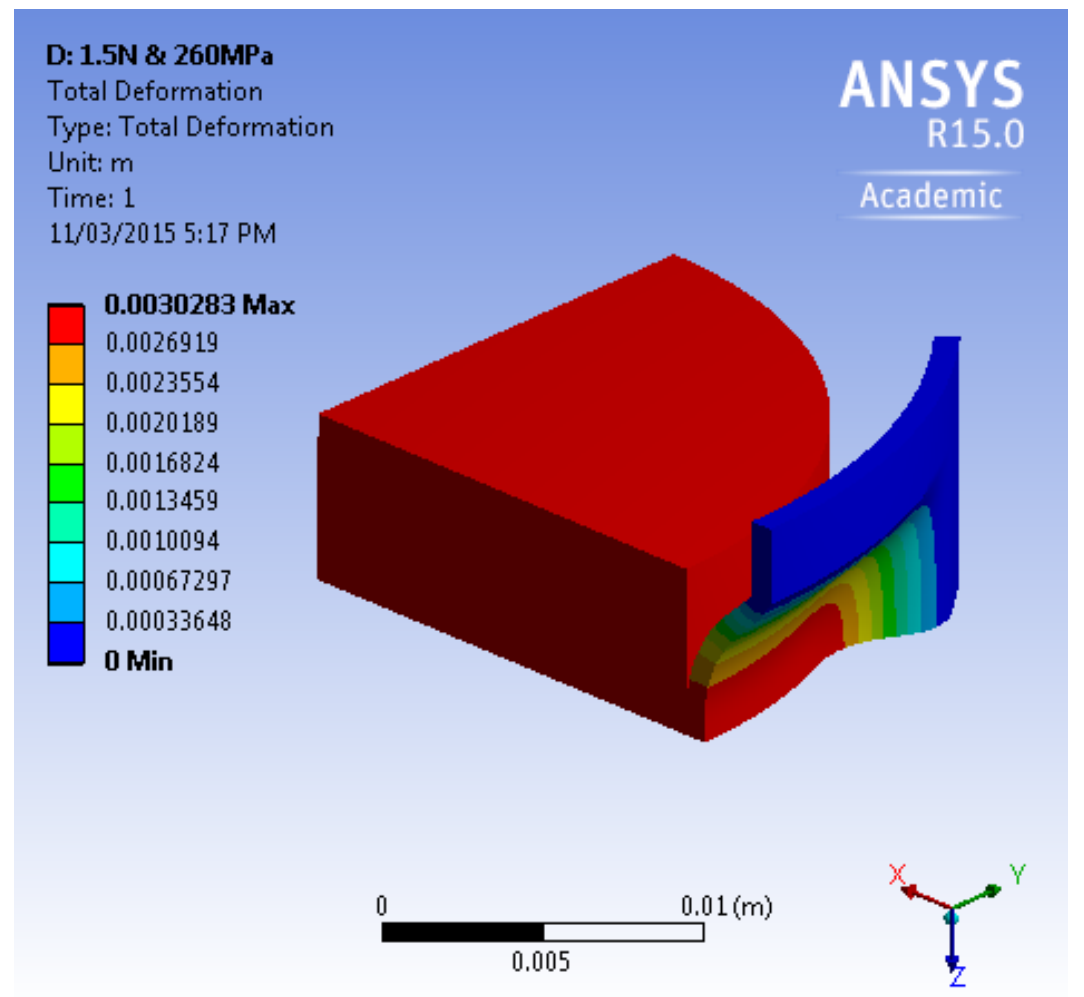

Figure A-8 Results for $1.5 \mathrm{~N}$ shaft force and $260 \mathrm{MPa}$ diaphragm pressure 


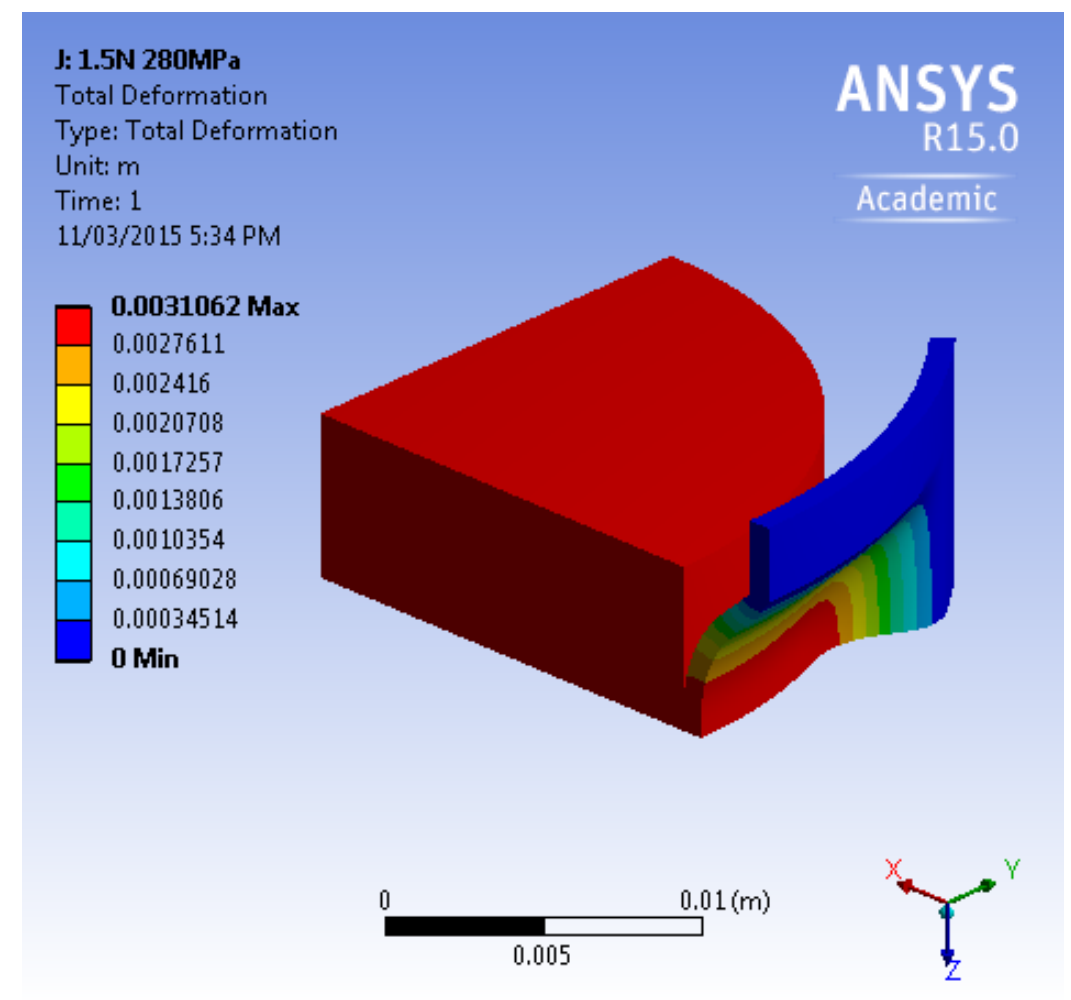

Figure A-9 Results for $1.5 \mathrm{~N}$ shaft force and 280MPa diaphragm pressure

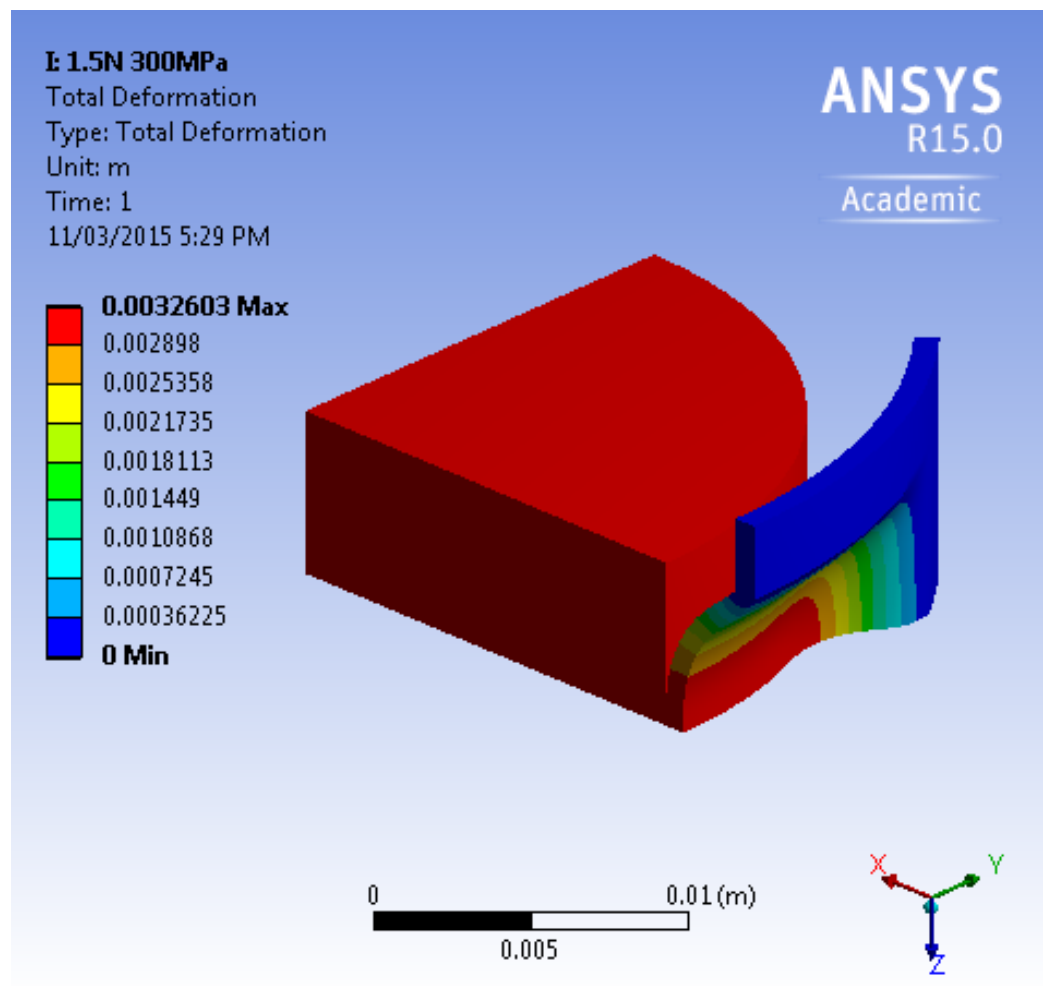

Figure A-10 Results for $1.5 \mathrm{~N}$ shaft force and 300MPa diaphragm pressure 


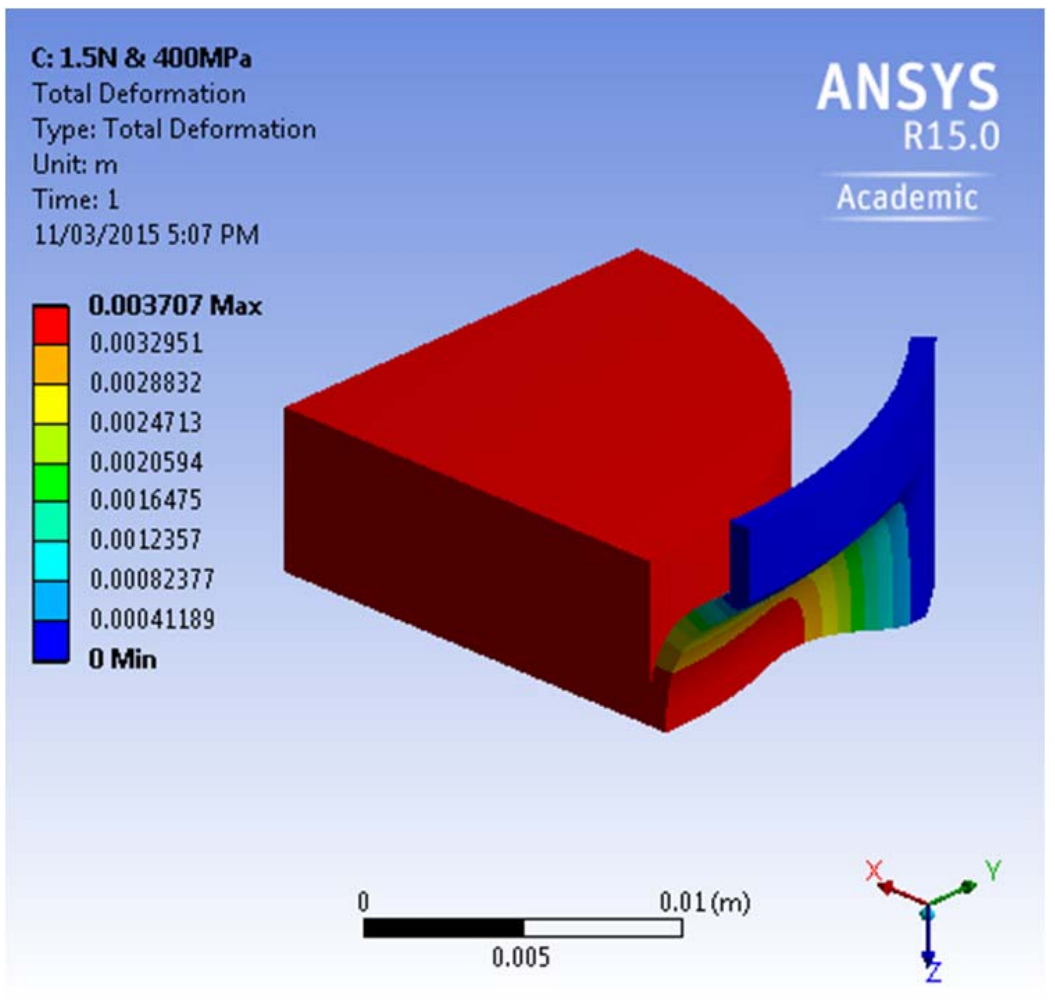

Figure A-11 Results for $1.5 \mathrm{~N}$ shaft force and 400MPa diaphragm pressure

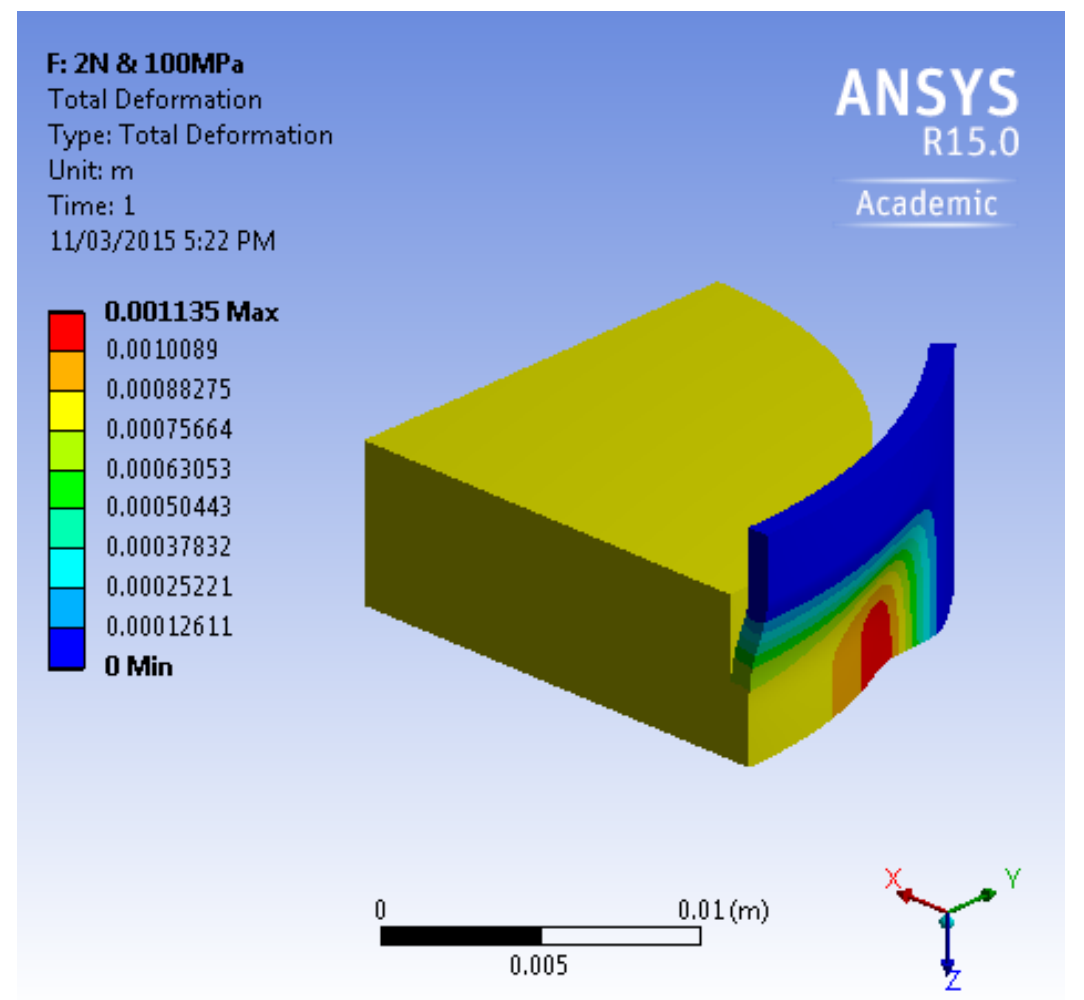

Figure A-12 Results for $2 \mathrm{~N}$ shaft force and 100MPa diaphragm pressure 


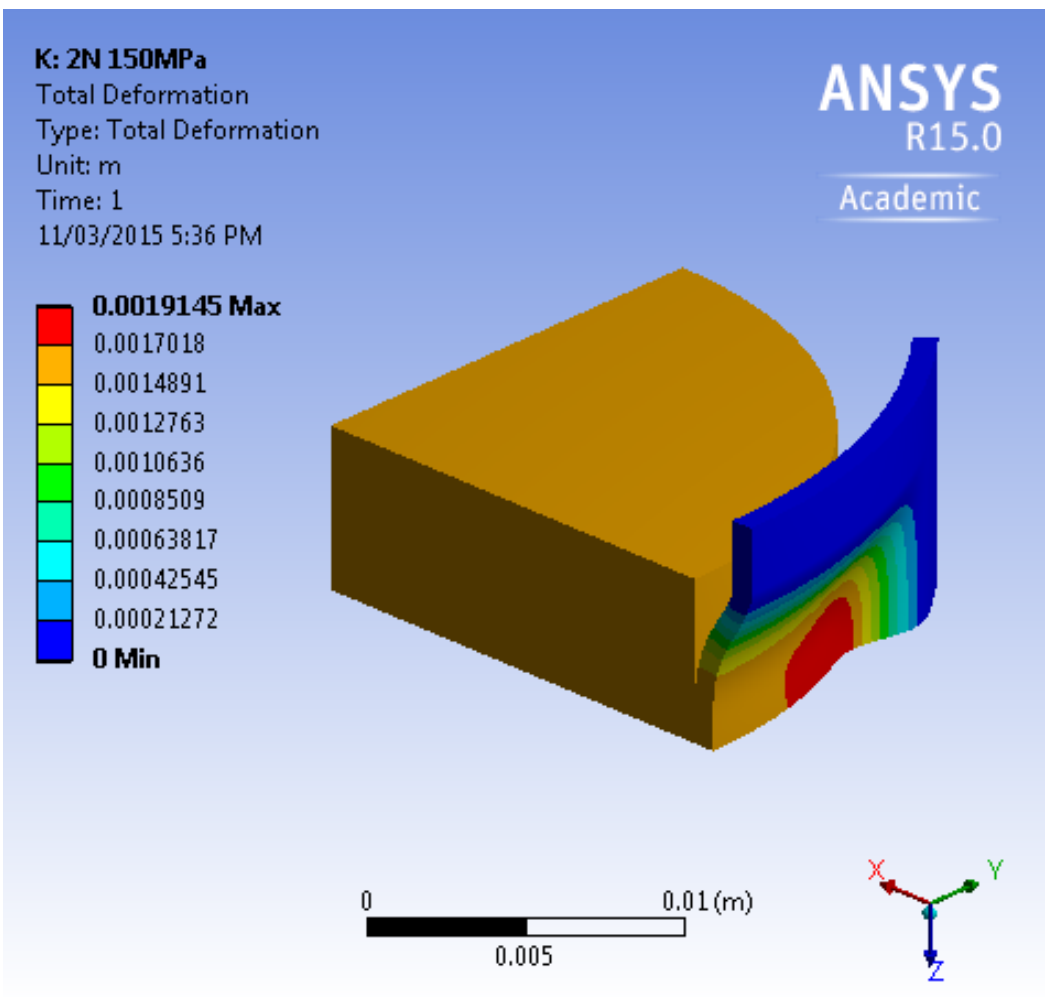

Figure A-13 Results for $2 \mathrm{~N}$ shaft force and $150 \mathrm{MPa}$ diaphragm pressure

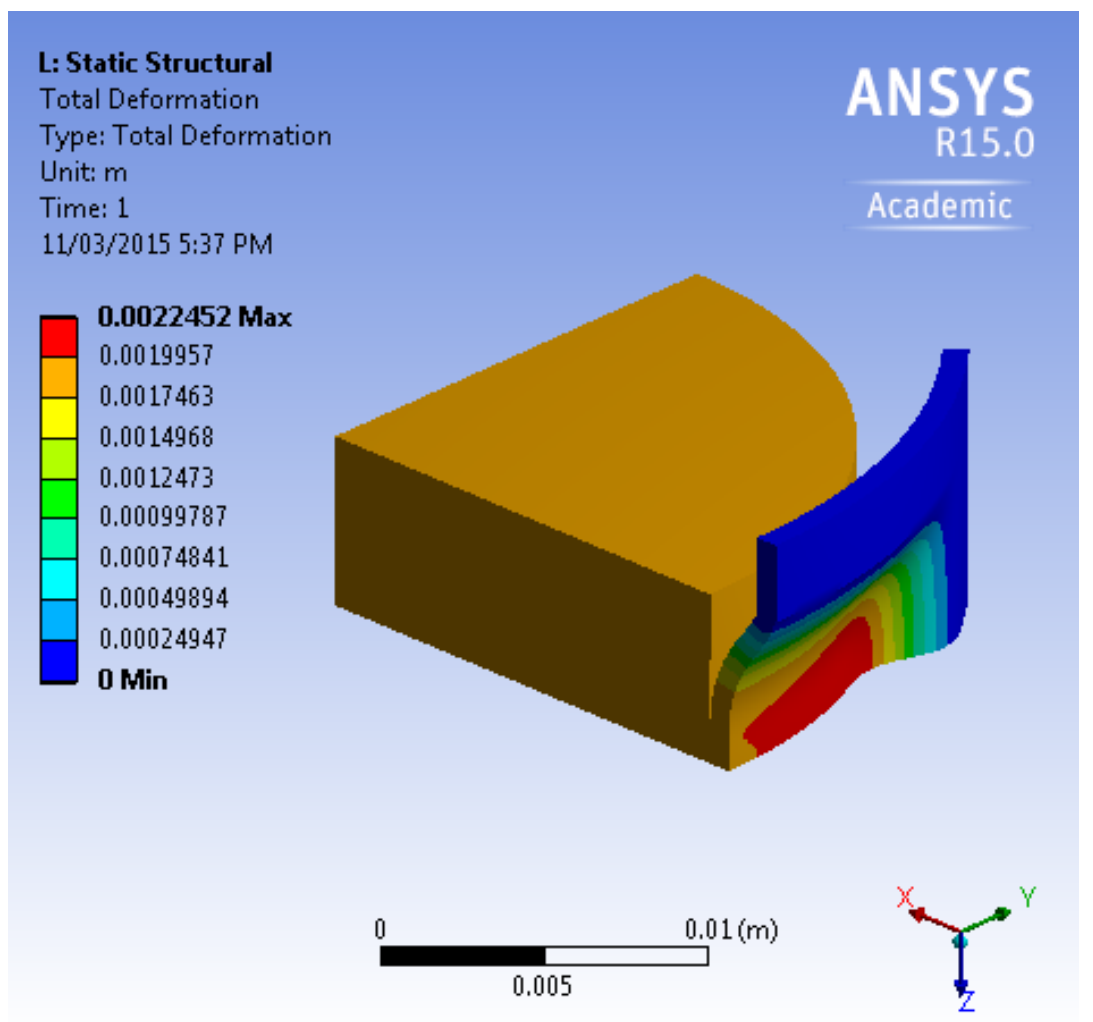

Figure A-14 Results for $2 \mathrm{~N}$ shaft force and $180 \mathrm{MPa}$ diaphragm pressure 


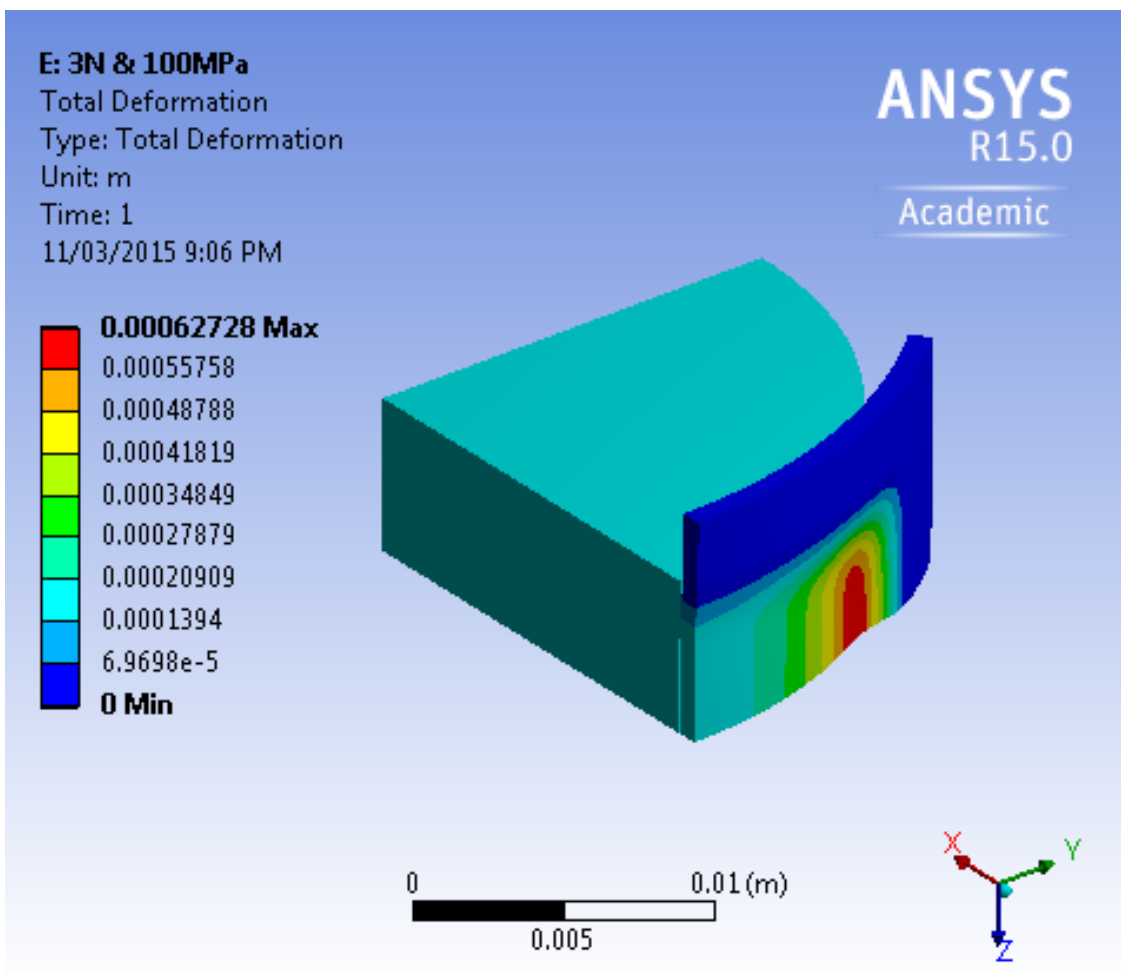

Figure A-15 Results for $3 \mathrm{~N}$ shaft force and $100 \mathrm{MPa}$ diaphragm pressure

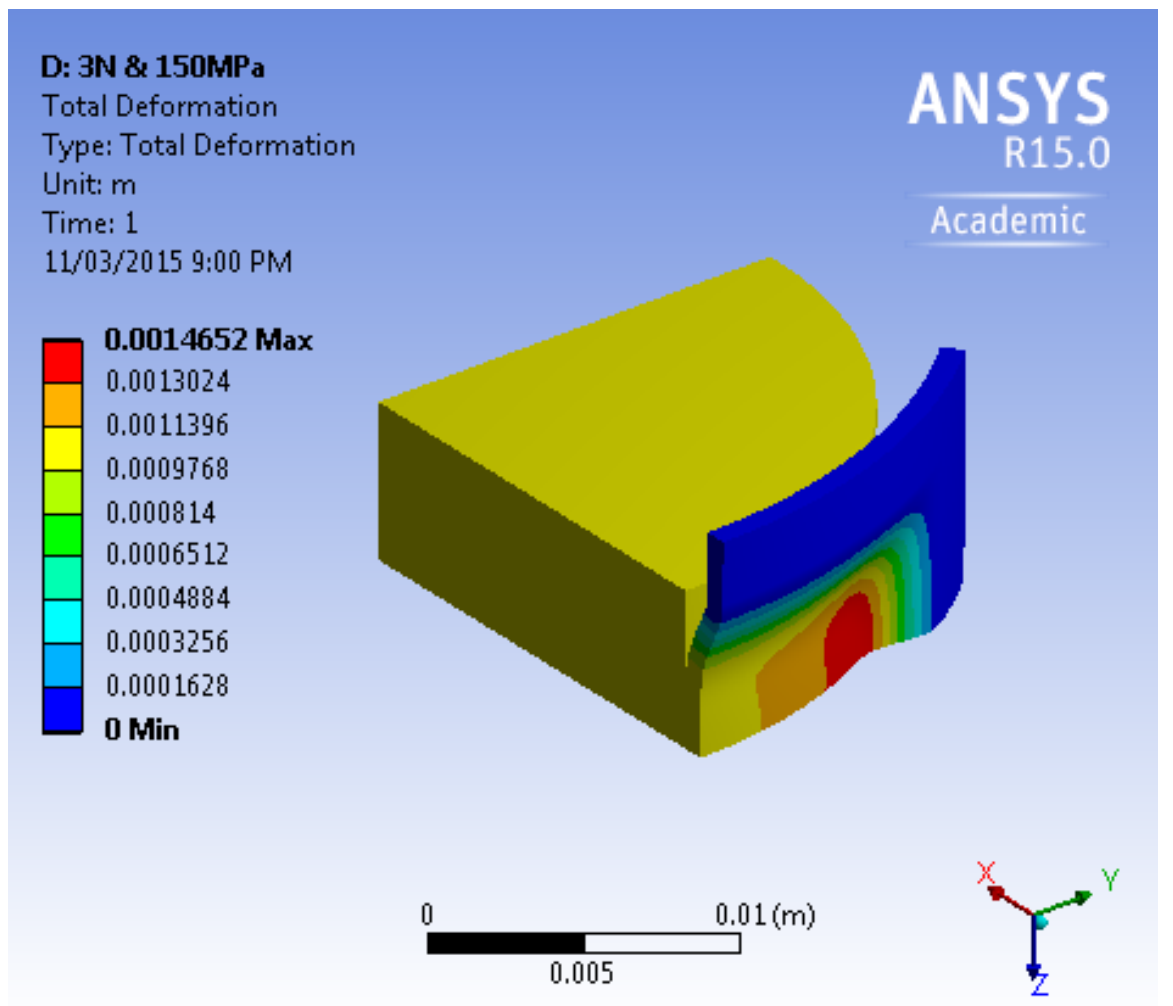

Figure A-16 Results for $3 \mathrm{~N}$ shaft force and 150MPa diaphragm pressure 


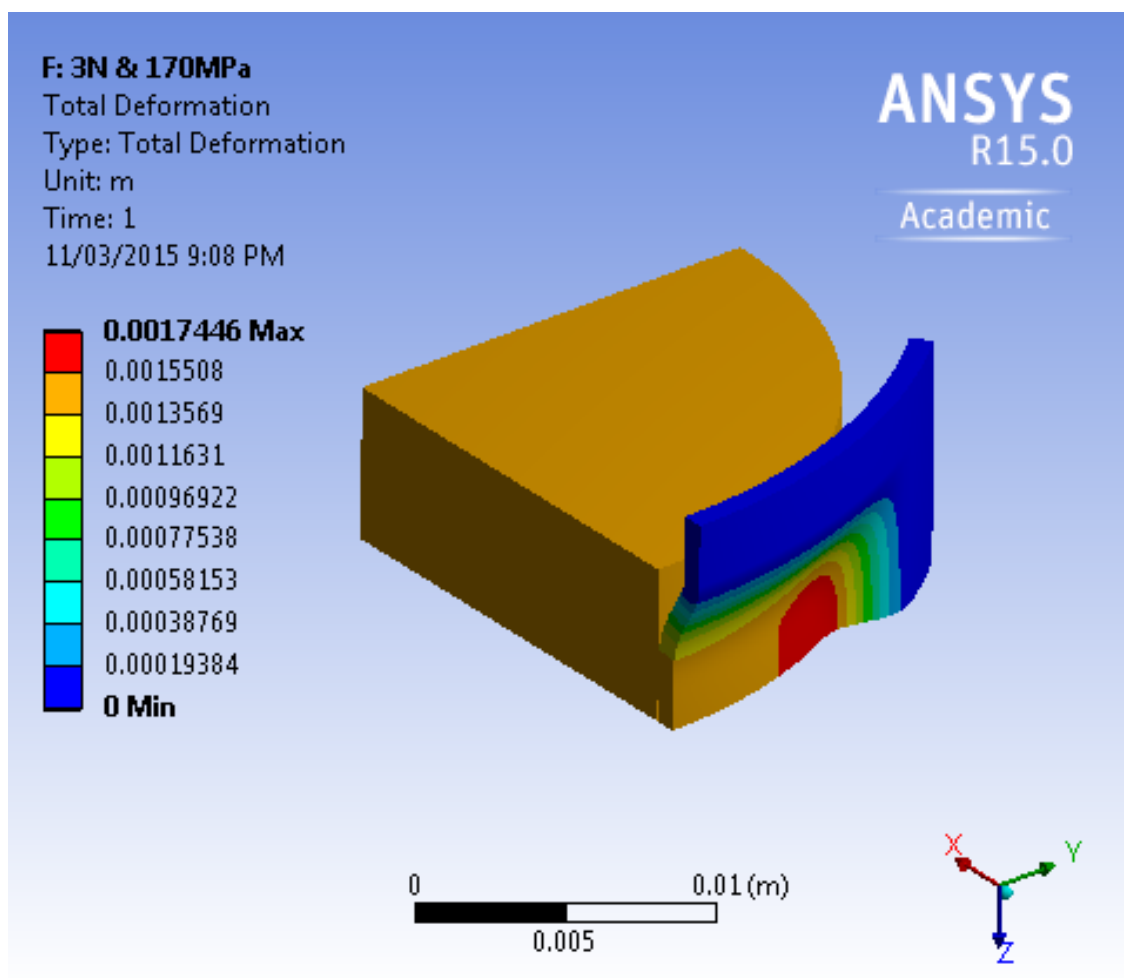

Figure A-17 Results for $3 \mathrm{~N}$ shaft force and $170 \mathrm{MPa}$ diaphragm pressure 


\section{Appendix B Electronic Hardware}

\section{Electronic Power Hardware}

Power to the sensing and data acquisition equipment was provided by a Mean Well DR-30 DC power supply. The supply provided approximately $24 \mathrm{VDC}$ to the $\mathrm{x}$ and $\mathrm{y}$ magnetic sensors and the data acquisition in parallel. The DR-30 was provided with $120 \mathrm{~V}$ AC wall power.

Terminal Pin No. Assignment
\begin{tabular}{|c|l|c|l|}
\hline Pin No. & Assignment & Pin No. & Assignment \\
\hline 1 & AC/N & 5,6 & -V \\
\hline 2 & AC/L & 7 & LED \\
\hline 3,4 & $+\mathrm{V}$ & 8 & +VADJ. \\
\hline
\end{tabular}
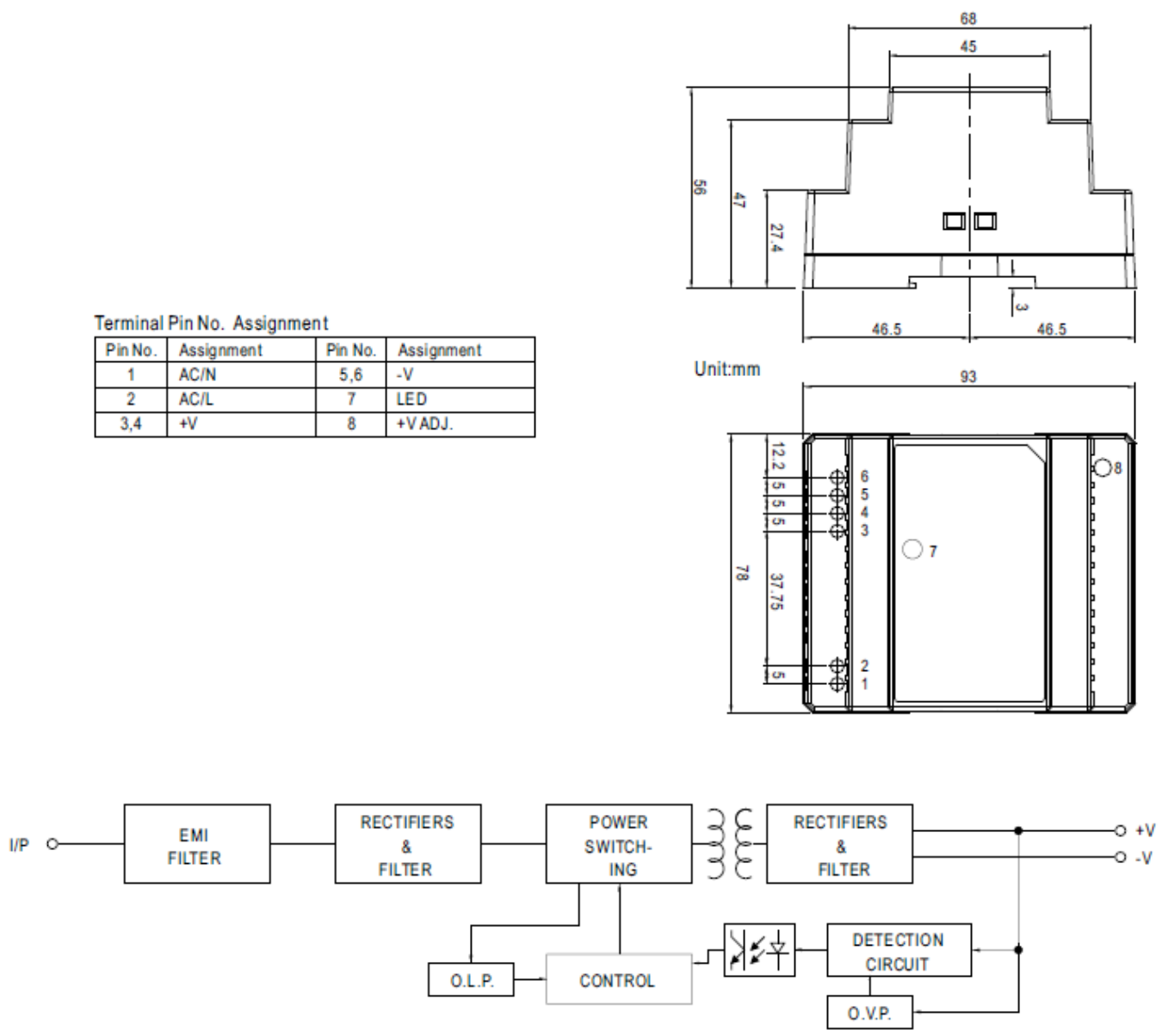

Figure B-1 Mean Well DR-30 Schematics [24] 


\section{Electronic Position Sensing Hardware}

The electronic position sensing hardware used during calibration and testing of the ERA was a custom made printed circuit board and integrated HMC1501 magnetic field sensor was designed and manufactured by Mr. Primoz Crensnik and Mr. Brian Petz for Petz's work [12]. All calibration and testing was completed using the previous design. Notable features of the design include:

- A 10V bridge supply

- $5 \mathrm{MHz}$ bandwidth

- HMC1501 sensors output +/-120mV has been amplified 39x for a $0.32 \mathrm{~V}$ to $9.68 \mathrm{~V}$ range

- Offset trimming is available for each sensor built into the hardware

- The $\mathrm{x}$ and $\mathrm{y}$ magnetic sensing unit requires a 12VDC power supply

- HMC1501 sensors have a resolution less than $0.07^{\circ}$
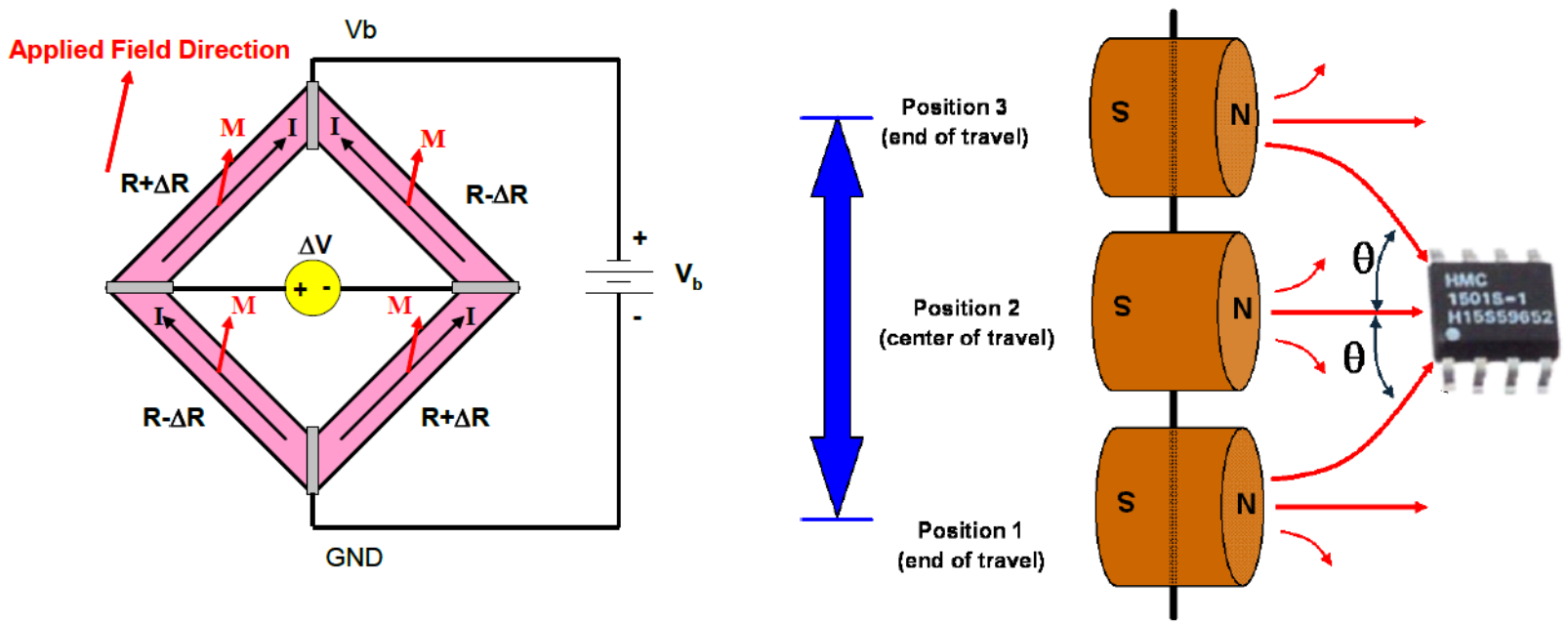

Figure B-2 HMC1501 magnetic sensor schematic [20] 
Schematic of Electronic Position Sensing Hardware

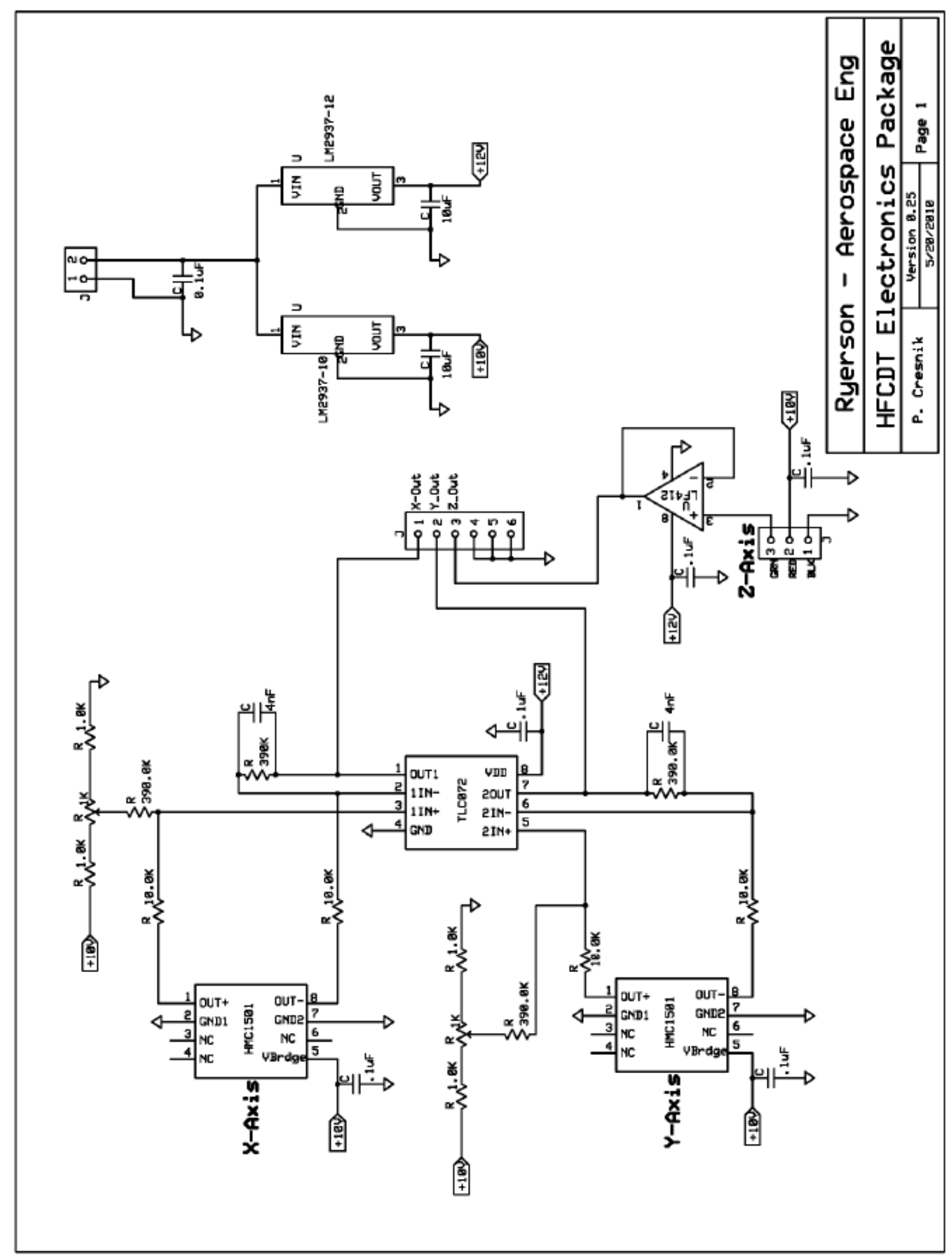

Figure B-3 HFCDT position sensing hardware schematics [12] 


\section{Electronic Pressure Sensing Hardware}

An Omega PX319-100A5V pressure transducer sensing was used in the testing phase to collect accurate pressure values for stiffness vs. pressure plots. Power is supplied to the unit via the data acquisition unit. The pressure transducer's output range was $0-5 \mathrm{~V}$ with a pressure range of 0 100psi. A direct linear relationship relates to the output range and the pressure range. This would make the output to pressure relationship 20psi/V. The resolution of the transducer is only limited to the data acquisition bandwidth.

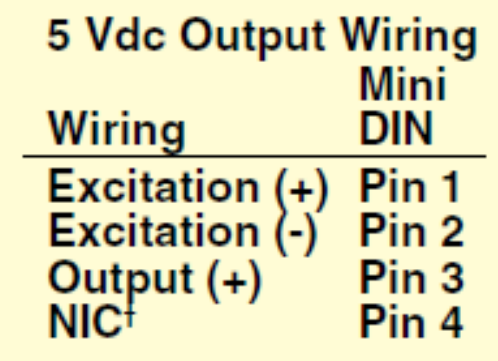

Electronic Data Acquisition Hardware

The USB-1208FS from Measurement Computing was used for all data acquisition. The unit was linked with Simulink in Matlab for programming. The unit was provided 18VDC and has a 5VDC output terminal for sensor excitation which was specifically used on the pressure transducer. The unit has a maximum continuous aggregated scan rate of $50 \mathrm{kS} / \mathrm{s}$. The total acquisition rate for all channels cannot exceed $50 \mathrm{kS} / \mathrm{s}$, therefore for calibration and testing 3 channels were used and the maximum sample rate was $16 \mathrm{kS} / \mathrm{s}$ for each channel. The unit was configured for single ended mode which means that each analog input has 11-bits of resolution. 


\section{Schematics for Electronic Data Acquisition Hardware}

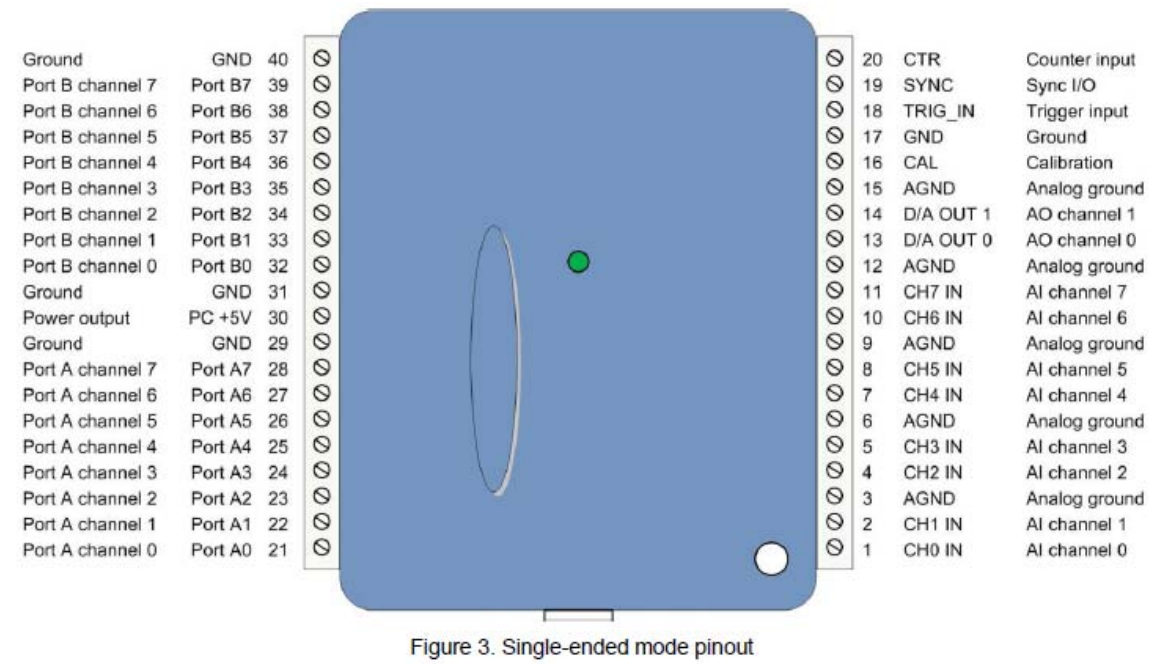

Figure B-5 Measurement Computing USB120FS pin location [26]
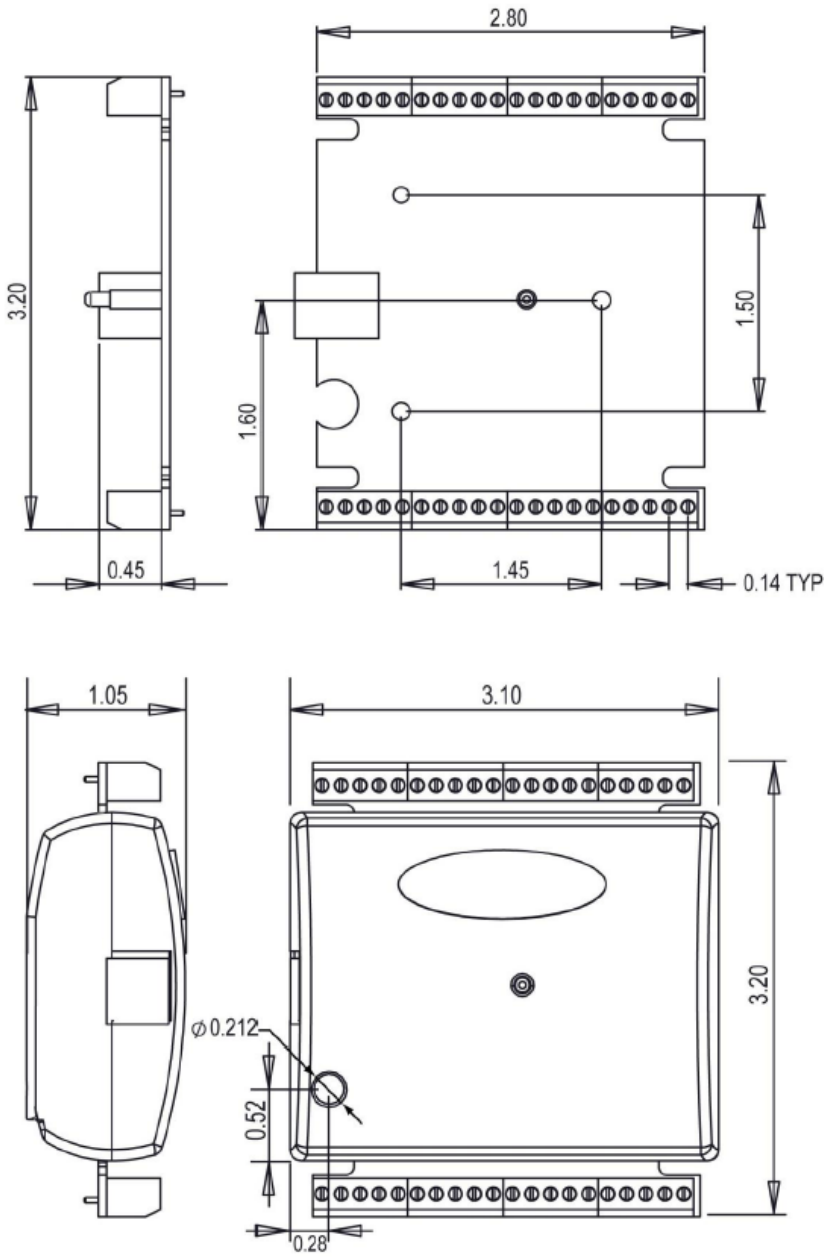

Figure B-6 Measurement Computing USB120FS schematics [26] 


\section{Appendix C Hyperelastic Material Data Information}

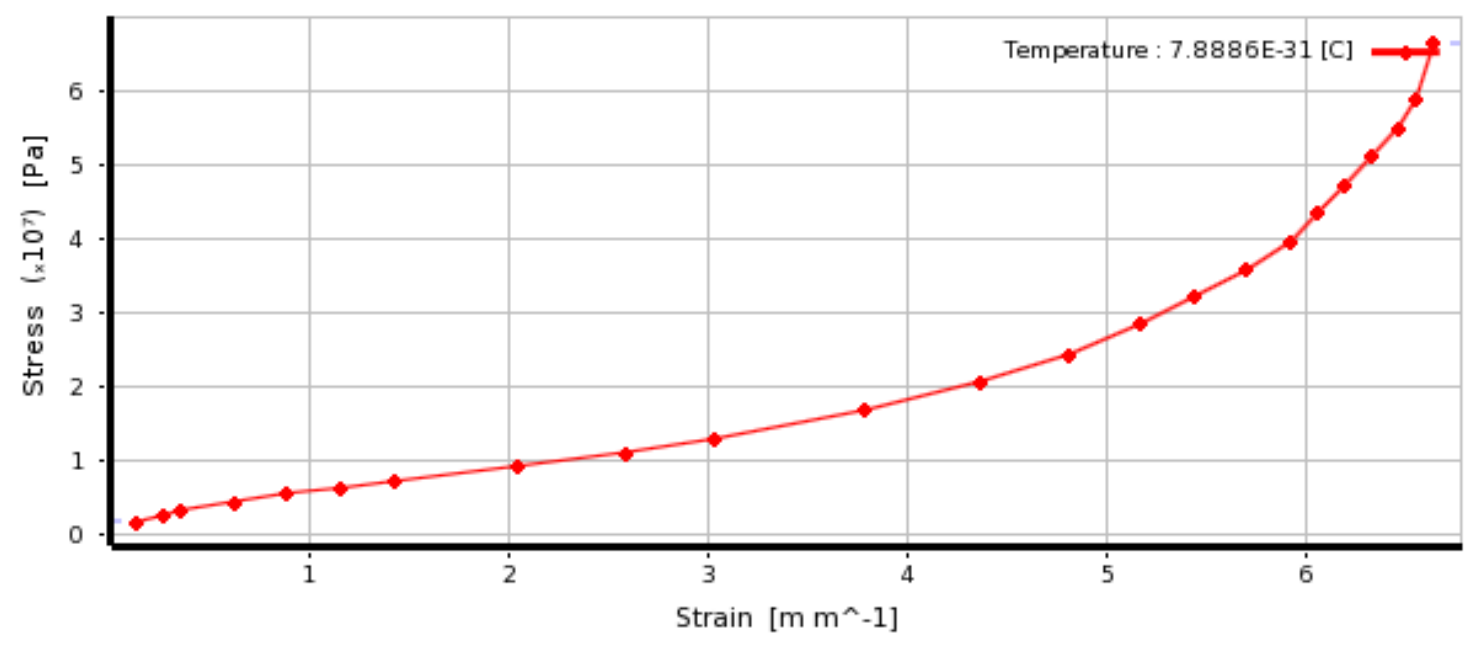

Figure C-1 Uniaxial test data from ANSYS

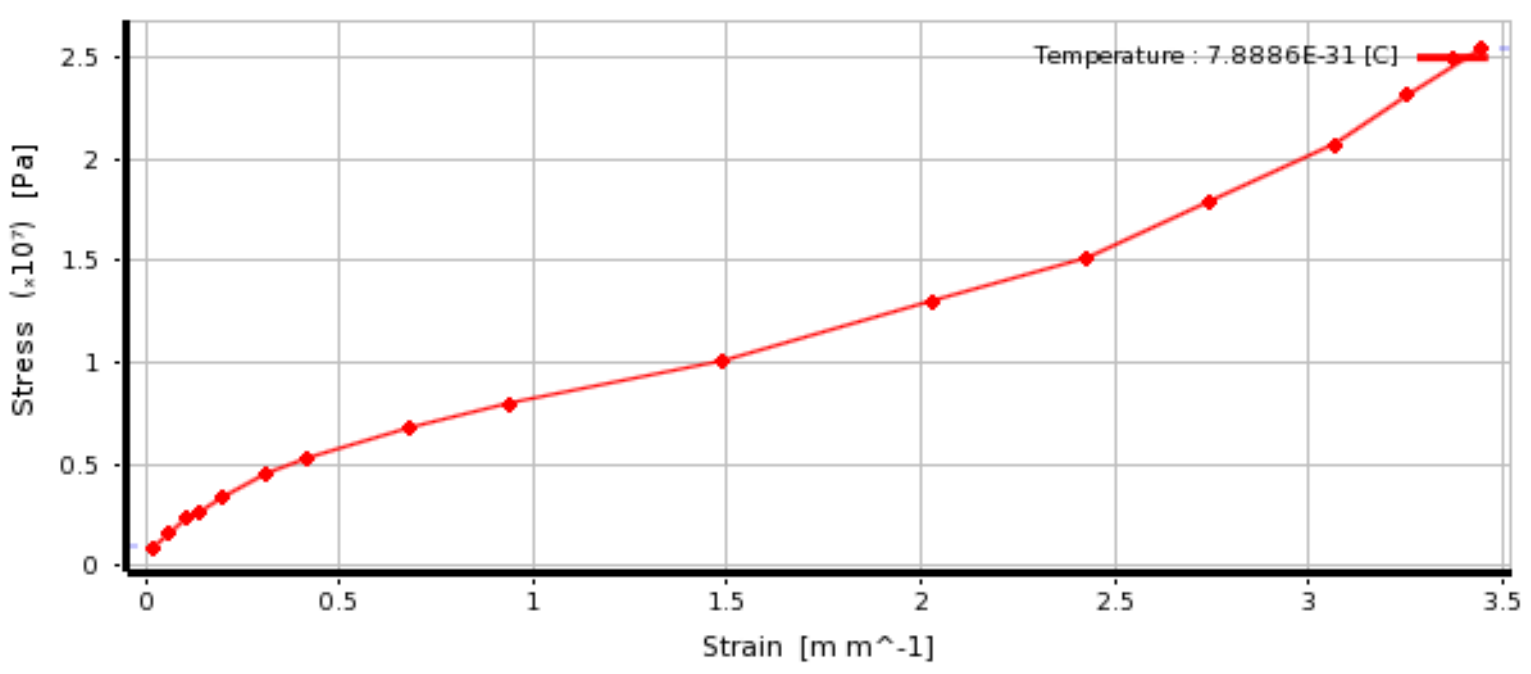

Figure C-2 Biaxial test data from ANSYS 


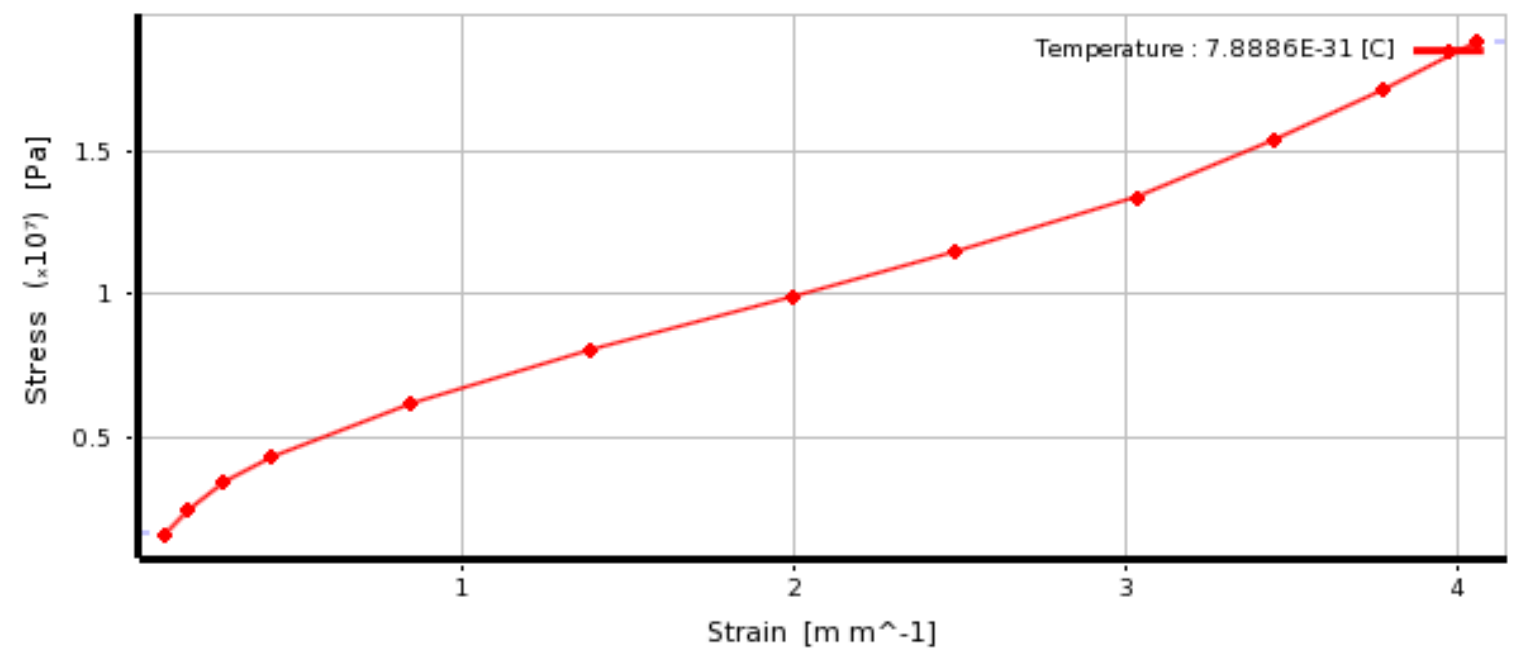

Figure C-3 Shear test data from ANSYS

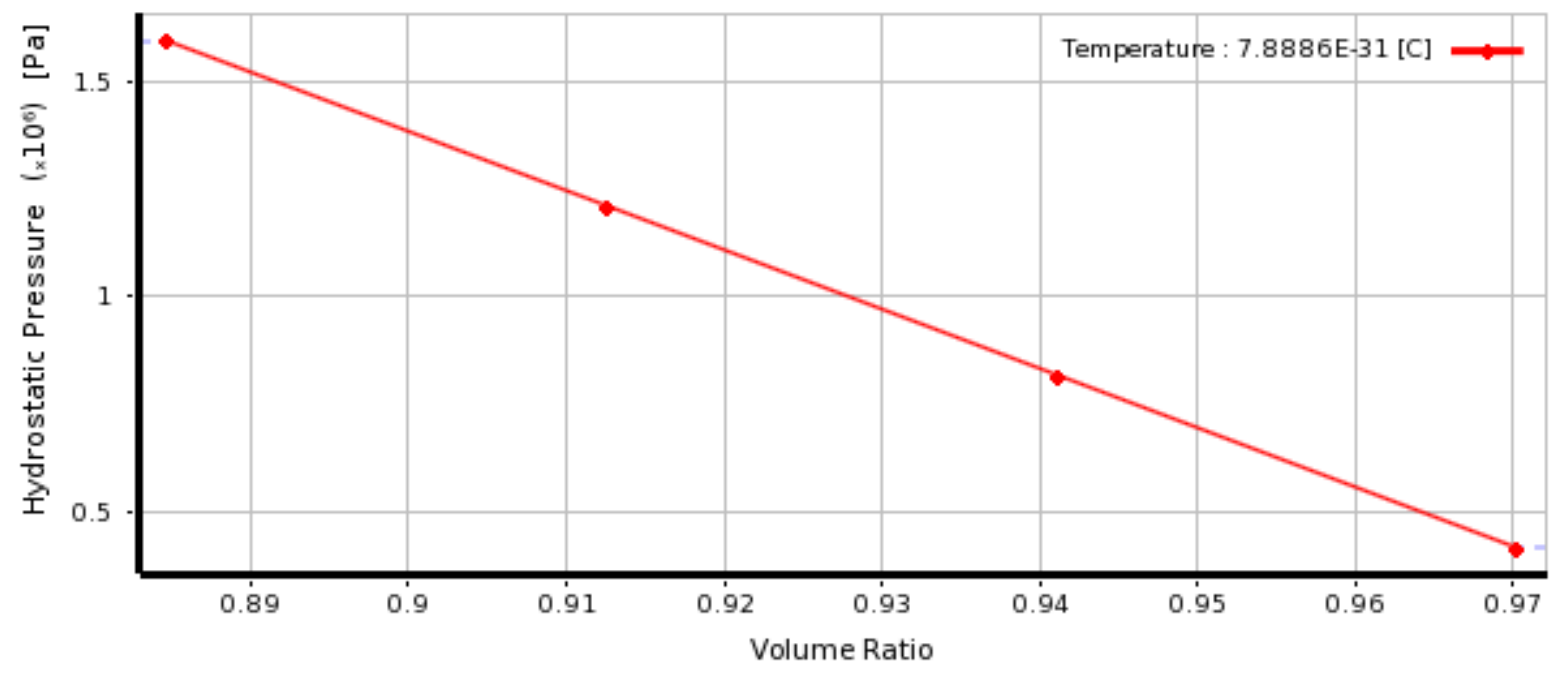

Figure C-4 Volumetric test data from ANSYS 